\title{
DISCLAIMER
}

This report was prepared as an account of work sponsored by an agency of the United States Government. Neither the United States Governinent nor any agency thereof, nor any of their employees, makes any warranty, express or implied, or assumes any legal liability or responsibility for the accuracy, completeness, or usefulness of any information, apparatus, product, or process disclosed, or represents that its use would not infringe privately owned rights. Reference herein to any specific commercial product, process, or service by trade name, trademark, manufacturer, or otherwise does not necessarily constitute or imply its endorsement, recommendation, or favoring by the United States Government or any agency thereof. The views and opinions of authors expressed herein do not necessarily state or reflect those of the United States Government or any agency thereof.
Distribution Category: Magnetic Fusion Energy (UC-20)

ANL/FPP/TM--213

DE87 007948

ARGONNE NATIONAL LABORATORY

9700 S uth Cass Avenue

Argonne, I1linois 60439-4837

\section{NONINDUCTIVE CURRBNT DRIVE FOR INTOR: A COMPARISON OF FOUR DRIVER OPTIONS}

\author{
by \\ David A. Ehst, Kenneth Evans, Jr. \\ Argonne National Laboratory \\ David R. Mikke1sen, David W. Ignat \\ Princeton Plasma Physics Laboratory \\ Tak Kuen Mau \\ University of California, Los Angeles
}

January 1987

\author{
Work supported by \\ .... \\ Office of Fusion Energy \\ U.S. Department of Energy \\ Under Contract W-31-109-ENG-38
}




\section{TARLE OF CONTBNTS}

Abstract

1. Four Driver Options................................. 1

2. Steady state.........................................4

2.1 Figure of Merit and Sensitivity to Plasma Parameters.......... 4

2.2 LH Current Drive................................. 5

2.3 HFFW Current Drive................................ 17

2.4 LFFW Current Drive................................ 34

2.5 NB Current Drive...................................48

2.6 Epitome of Steady state Calculations.................... 57

3. Current Density Profile Control............................ 57

3.1 Value of Current Density Control........................ 57

3.2 LH Current Tailoring................................ 59

3.3 HFFW Current Talloring............................... 59

3.4 LFFW Current Tailoring............................. 62

3.5 NB Current Talloring............................. 67

4. Current Drive with Reversed Electric Field..................... 69

4.1 Theoretical Model for Wave-Driven Currents.................69

4.2 LH Current Drive................................... 72

4.3 Fast Wave Current Drive............................. 74

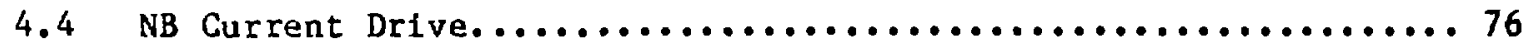

4.5 Epltome of Electric Field Effects...................... 78

5. Conclusions.........................................80

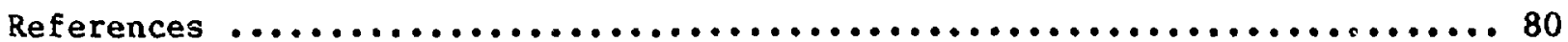




\section{LIST OR TABLES}

No.

Page

I

II

I I I

IV

v

VI

VII

VIII

IX

$\mathrm{X}$

XI

$X I I$

Geometry and Plasma............................... 3

Previous $\gamma$ Calculations for LHCD...................... 6

Previous $\gamma$ Calculations for HFFW.................... 18

HFFW Current Drive Sensitivity to Impurity Content, Plasma

Profiles and Ion Temperature........................... 31

Previous $\gamma$ Predictions for LFFW...................... 38

Previous $\gamma$ Predictions for NB....................... 52

Tokamak, Plasma, and Beam Description...................... 55

NB-Driven Steady State............................ 56

X Summary of Steady State Benchmark Calculations.............. 58

Shell Currents with NB............................68 68

XI NB Current Drive with Reversed Electric Fleld.............. 77

Summary of Electric Field Ef fects $(E=-0.01 \mathrm{~V} / \mathrm{m}) \ldots \ldots \ldots \ldots \ldots . .79$

\section{LIST OF FIGURES}

No.

$\underline{\text { Page }}$

1 Experimental $\gamma=\bar{n}_{e} I R_{o} / \operatorname{Pinj}\left(10^{20} \mathrm{~m}^{-3}-M A-m / M W\right)$ for steady state lower hybrid current drive with $v_{10 o p}=0$ and $t=0$. Pinj is total injected power, ignoring directivity and accessi bility. Dashed lines are theoretical values, assuming total absorption of injected power.

2a Ray path for $\mathrm{LH}$ wave at $\mathrm{f}=5.0 \mathrm{GHz}, \mathrm{n}_{\mathbf{l}}=1.6$, in a plasma with $\overline{\mathrm{T}}_{\mathrm{e}}=20 \mathrm{keV}, \mathrm{T}_{\mathrm{eo}}=35 \mathrm{keV}, \mathrm{n}_{\mathrm{eo}}=1.23 \times 10^{20} \mathrm{~m}^{-3}, \mathrm{~T}_{10}=18 \mathrm{keV}$, $B_{0}=5.5 \mathrm{~T}$, and $R_{0}=4.9 \mathrm{~m}$.

2b Damping of wave in Fig. 2a. 10

3a Same as Fig. 2 except $\overline{\mathrm{T}}_{\mathrm{e}}=10 \mathrm{keV}, \mathrm{T}_{\mathrm{eo}}=18 \mathrm{keV}, \mathrm{T}_{10}=35 \mathrm{keV}$, and $n_{\|}=1.8$. Abscissa for $n_{1}$ and $n_{\perp}$ plots is $n_{e} / n_{e 0}$ 


\section{LIST OF PIGURES (Continued)}

No.

Page

3b Wave penetrates further without damping as $\overline{\mathrm{T}}_{\mathrm{e}}$ 1s lowered........12

$4 a$ Ray path for $\mathrm{LH}$ wave at $\mathrm{f}=10 \mathrm{GHz}, \mathrm{n}_{\|}=1.9$, In plasma with

$\overrightarrow{\mathrm{T}}_{\mathrm{e}}=5 \mathrm{keV}, \mathrm{T}_{\mathrm{eo}}=8.8 \mathrm{keV}, \mathrm{n}_{\mathrm{eo}}=1.23 \times 10^{20} \mathrm{~m}^{-3}, \mathrm{~T}_{\mathrm{io}}=26 \mathrm{keV}$,

$\mathrm{B}_{\mathrm{o}}=5.5 \mathrm{~T}$, and $\mathrm{R}_{\mathrm{o}}=4.9 \mathrm{~m} \ldots \ldots \ldots \ldots \ldots \ldots \ldots \ldots \ldots \ldots \ldots \ldots \ldots \ldots \ldots$

$4 \mathrm{~b}$ Wave damps nearer the magnetlc axls as $\overline{\mathrm{T}}_{\mathrm{e}}$ is reduced to $\sim 5 \mathrm{keV} \ldots 15$

5 LH equilibria with multiray spectrum, $5 \leq \mathrm{f}(\mathrm{GHz}) \leq 10,1.8 \leq$

$\mathrm{n}_{\|} \leq 4.0$, with total power $\mathrm{P}_{\mathrm{CD}}=87 \mathrm{MW}$ : (a) has $40 \%$ current

averaging, $\bar{n}_{e}=0.73 \times 10^{20} \mathrm{~m}^{-3}, \beta_{t}=0.0506, I_{0}=8.2 \mathrm{MA}$, and

$\gamma=0.34$; (b) is similar result with $60 \%$ averaging and $\gamma=0.30 \ldots \ldots 16$

6a Normalized $\tilde{\mathrm{j}} / \tilde{p} r f$ : analytic expression (curves) compared to specific evaluations. Solid curve assumes Landau damping, dashed assumes TTMP.

6b Normalized $\tilde{J} / \tilde{p} r f$ with $z_{\text {eff }}=2$. Abscissa is $w=\omega / k{ }_{\|} e$, where $v_{e}=\sqrt{T_{e} / m_{e}}$

7 INTOR benchmark - equilibrium generated by HFFW with $\overrightarrow{\mathrm{n}}_{\mathrm{e}}=0.7$

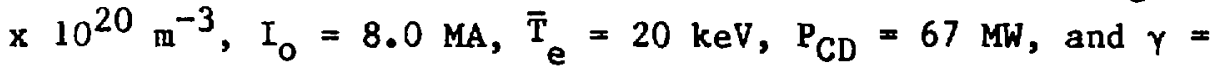
0.41 . Spectrum has $0.3 \leq \mathrm{f}(\mathrm{GHz}) \leq 1.0,1.6 \leq \mathrm{n}_{\|} \leq 2.8$; additlonal power $(\lesssim 1 \mathrm{MW})$ is provided as $3.0 \mathrm{GHz} \mathrm{LH}$ waves...

8 Current drive efficiency versus electron density with other parameters flxed; $R_{0}=4.90 \mathrm{~m}, I=8.0 \mathrm{MA}, A=4.2, K=1.6$, $\alpha=1.4, B_{0}=5.5 \mathrm{~T}, \mathrm{z}_{\text {eff }}=1.5, \alpha_{\mathrm{n}}=\alpha_{\mathrm{T}}=0.7$, $\overline{\mathrm{T}}_{\mathrm{e}}=20 \mathrm{keV}, \overline{\mathrm{T}}_{1}=10 \mathrm{keV}$. 


\section{LIST OF PIGURES (Contimued)}

No.

Page

9 Equilibrium generated by HFFW for full density INTOR: $\overline{\mathrm{n}}_{\mathrm{e}}=1.4$ $\times 10^{20} \mathrm{~m}^{-3}, I_{0}=8.0 \mathrm{MA}, \overline{\mathrm{T}}_{\mathrm{e}}=20 \mathrm{keV}, \mathrm{P}_{\mathrm{CD}}=117 \mathrm{MW}$, and $\gamma=$ 0.47. Note that the resulting $B_{t}=0.071$ at this high $\overrightarrow{\mathrm{T}}_{e}$ value exceeds the Troyon 1 imit................................ 27

10 Current drive power versus major radius; $B_{t}=0.0582, A=4.5$, $\kappa=1.6, \alpha=1.4, Z_{\text {eff }}=1.5, \alpha_{n}=1.1, \overline{\mathrm{T}}_{\mathrm{e}}=18 \mathrm{keV}, \overline{\mathrm{T}}_{1}=12$ $\mathrm{kev}, \mathrm{G}_{\mathrm{n}}=3.5 \mathrm{MW} / \mathrm{m}^{2}$.

11 Current drive efficlency versus electron temperature; $R_{0}=$ $5.68 \mathrm{~m}, \dot{\beta}_{t}=0.0409, A=6.0, k=1.6, \alpha=1.4, z_{\text {eff }}=1.5$, $\alpha_{n}=1.1, \bar{n}_{e} \simeq 2.3 \times 10^{20} \mathrm{~m}^{-3}, \bar{T}_{1}=11 \mathrm{keV}, \omega_{n}=3.5 \mathrm{MW} / \mathrm{m}^{2}$

12 First stability reglme limits: solid curves - Troyon formula; dashed curve - Bernard scallng. In all cases $A=6.0, \alpha=1.4$, $q_{a}=1.0$, and $q_{b}=2.0$. Triangles show equilibrla duplicated by HFFW current drive calculations. Insets display $J_{t}(R, 0)$.

13 Current drive efficiency versus elongation; $A=6.0, \alpha=1.4$, $z_{\text {eff }}=1.5, a_{n}=1.1, \bar{T}_{e}=18 \mathrm{keV}, \bar{T}_{1}=12 \mathrm{keV}, W_{n}=3.5 \mathrm{MW} / \mathrm{m}^{2}$, $\mathrm{P}_{\mathrm{f}}=1330 \mathrm{MW}$.

14 Current drive efficiency versus aspect ratio; $k=1.6, \alpha=1.4$, $Z_{\text {eff }}=1.5, \alpha_{n}=1.1, \bar{T}_{e}=18 \mathrm{keV}, \bar{T}_{1}=12 \mathrm{keV}, w_{n}=3.5 \mathrm{MW} / \mathrm{m}^{2}$, $P_{f}=1330 \mathrm{MH}$.

15 Universal current drive efficiency: $\alpha=1.4, \mathrm{z}_{\text {eff }}=1.5, \alpha_{\mathrm{n}}=$ i. $1, \overline{\mathrm{T}}_{\mathbf{i}} \simeq 12 \mathrm{keV}$ 


\section{LIST OF PIGURES (Contimued)}

No.

Page

17 Ray paths for a broad poloidal mode spectrum launched from the antenna in Fig. 16. Crosses denote 5\% damping decrements.......4 41

18 Wave radial power dissipation; bottom curve is power absorbed by electrons, remaining increments are to tritium and beryllium.... 42

19 Current density profile for HSMS at $82 \mathrm{MHz}$ and $\lambda_{\|} \approx 0.8 \mathrm{~m} \ldots \ldots \ldots \ldots$

20 Typical ray history for benchmark LFFW: $f=64 \mathrm{MHz}, \mathrm{n}_{\|}=3.5$, $\mathrm{T}_{\text {eo }}=35 \mathrm{keV}, \mathrm{n}_{\text {eo }}=1.23 \times 10^{20} \mathrm{~m}^{-3}$, and $\mathrm{T}_{10}=18 \mathrm{keV}$. Note spatial locations of lon resonances for $A=4.2, B_{0}=5.5$ T..... 44

21 Same ray as in Fig. 20. Ion damping only occurs when the ray reaches the $\omega=2 \Omega_{\mathrm{T}}$ radius, but over $90 \%$ of the wave power has already been dissipated on electrons by that time............45

22 Benchmark INTOR with LFFW equilibrium generated with $f=64$ MHz, $3.0 \leq \mathrm{n}_{\mathrm{H}} \leq 4.5, \mathrm{P}_{\mathrm{CD}}=52 \mathrm{MW}, \mathrm{I}_{\mathrm{o}}=5.9 \mathrm{MA}, \overline{\mathrm{n}}_{\mathrm{e}}=0.59 \times 10^{20} \mathrm{~m}^{-3}$, $\overline{\mathrm{T}}_{\mathrm{e}}=17 \mathrm{keV}$, and $B_{t}=0.031$, for which $\gamma=0.33 \ldots$

$23 D^{\circ}$ injection into $D-T$ plasma with $Z_{\text {eff }}=2$

25 Equilibrium beta and toroldal current for various peak densities and pressures; all other parameters are those of the reference case, * except $\mathrm{T}_{\mathrm{DO}}=42.9 \mathrm{keV}$. Toroidal current density $\mathrm{j}$ and safety factor $q$, shown in the insets, vary along the curves.......60

$$
\begin{aligned}
& * R_{0}=5.25 \mathrm{~m}, A=6, k=1.6, B_{0}=3.7 \mathrm{~T}, \mathrm{~T}_{\text {eo }}=18 \mathrm{keV}, a_{n}= \\
& 1.05, \alpha_{T}=0.35, \mathrm{P}_{C D^{\prime}}=50 \mathrm{MW}, \mathrm{f}=0.4 \mathrm{GHz}, \mathrm{n}_{n}=2.4 .
\end{aligned}
$$




\section{LIST OF FIGURES (Contimued)}

No.

Page

26 Equilibrium beta and toroldal current for varlous fast wave frequencies; all other parameters are those of the reference case, * except $\mathrm{T}_{\text {eo }}=15.0 \mathrm{keV}, \mathrm{T}_{\mathrm{DO}}=15.5 \mathrm{keV}$ and $P_{\text {edge }}=60 \mathrm{MW}$. The toroldal current density is shown in the insets............61

See FIg. 25.

27 High beta equilibrium generated with the HFFW (plus a small amount of $\mathrm{LH}$ power to control the edge current density): $B_{t}=$ $0.079, I_{0}=3.2 \mathrm{MA}, B_{0}=3.66, R_{0}=5.25 \mathrm{~m}, \vec{n}_{e}=0.98 \times 10^{20}$ $\mathrm{m}^{-3}, \mathrm{~T}_{\mathrm{e}}=13 \mathrm{keV}$, and $\gamma=0.35 ; \mathrm{P}_{\mathrm{CD}}=47 \mathrm{MW}$

28 Pressure and poloidal flux across midplane for Fig. 27.......... 64

3la Current density profiles for neutral beam driven current with extended source of varying height above the midplane.

31b Current density profile with localized neutral beam source at different vertical positions.......................... 7

32 Factor multiplying $\gamma$ when $E \neq 0$. Normalized phase speed to runaway speed is $u_{r} \equiv c /\left(n_{n} v_{r}\right)$, where $v_{r} \equiv 9.3 \times 10^{-3} \sqrt{n_{e} / E}$ In SI units. During ramp-up the sign convention is $u_{r}>0 \ldots \ldots \ldots \ldots 73$

33 Benchmark INTOR for very 1 ow $\overline{\mathrm{n}}_{\mathrm{e}}\left(=0.04 \times 10^{20} \mathrm{~m}^{-3}\right)$ transformer recharge with $\mathrm{LH}$ waves: $\overline{\mathrm{T}}_{\mathrm{e}}=2 \mathrm{keV}, \gamma=0.10$, and centrally peaked current is obtained wth $f=5.0 \mathrm{GHz}$ and $2.4 \leq \mathrm{n}_{n} \leq 10.0 . \ldots 75$ 


\title{
MONINDUCTIVE CURRENT DRIVE FOR INTOR: A COMPARISON OF FOUR DRIVER OPTIONS
}

\author{
David A. Ehst, Kenneth Evans, Jr. \\ Argonne National Laboratory \\ David R. Mikke Isen, David W. Ignat \\ Princeton PI asma Physics Laboratory \\ Tak Kuen Mau \\ University of California, Los Angeles
}

\begin{abstract}
The current drive power and normalized efficiency are calculated for the INTOR tokamak, including spatial protiles of the plasma and current density. Current drive requirements are computed for purely steady state operation with no electromotive force and also in the presence of a reversed emf typical of start-up or transformer recharging. Results are obtained for lower-hybrid-waves, high frequencey fast waves, low frequency fast waves and neutral beam injection.
\end{abstract}

\section{FOUR DRIVER OPTIONS}

Largely as a result of assessments made at the Specialists: Meeting on Tokamak Concept Innovations (January, 1986) I four distinct drivers have been selected for analysis and benchmark calculations by the participants of the regular INTOR Workshop. As an introduction to the analysis, the characteristics of the four options will here be briefly:outlined.

The lower-hybrid (LH) wave typically has a frequenc: $f \geq 1 \mathrm{GHz}$ and is launched by an end-fire array of waveguides aligned so the wave electric field lies in the toroidal direction. The relative phasing of the guides is selected to drive a travelling wave with an index of refraction along the tokamak magnetic fleld with $1<n_{1} \leqslant 3$. In theory and in practice it appears difficult to generate LH current near the center of a hot ( $T_{e_{0}} \gg 1 \mathrm{keV}$ ), high density $\left(n_{e_{0}} \gtrsim 1 \times 10^{20} \mathrm{~m}^{-3}\right)$ tokamak plasma.

The high frequency fast wave (HFFW) also has $f \gtrsim 1 \mathrm{GHz}$ but has an electric field polarization in the poloidal direction. It could be launched 
with elther phased loop antennas or by properly folded or slutted wavegulde arrays. Theoretically this wave will propagate readily into the center of a reactor-grade plasma where current would be generated via Landau damping and transit-time magnetic pumping on the electrons.

The low frequency fast wave (LFFW) would typically have a frequency between the second ion cyclotron harmonics of deuterium and tritium i 50 - 100 $M H z)$ and could be launched by a phased array of loop antennas or ridged waveguides. Slight frequency tuning could allow a transition between ion harmonic heating (for reactor ignition) and electron damping (for current drive). In addition to direct electron damping of the fast wave it may be possible to generate current by electron damping of a mode-converted ion Bernsteln wave; ${ }^{2}$ alternatively, minority heating could generate current. 3

Neutral beam (NB) injection has successfully driven toroidal current, and good penetration into reactor densities is expected if high energy $\left(E_{b} \gtrsim 0.5\right.$ MeV) deuterium beams are developed. A neutral current on the order of $100 \mathrm{~A}$ would be necessary; this might require several tangential beam lines penetrating the tokamak assembly.

The physics status of these current drive options was addressed at a Specialists' Meeting on Noninductive Current Drive (September, 1986).4 It was noted at that meeting that the experimental data base is strong for LH current drive at low density, both with and without an emf, although extrapolation to reactor density and temperatures is problemmatic. Neutral bean drive has also enjoyed experimental demonstration to levels as high as $0.5 \mathrm{MA}$. However, the fast wave drivers have not yei recelved adequate experimental study, so their inclusion in our study is based mostly on the theoretical promise of current drive in reactor-like plasmas.

The goal of the present comparison study is, using the latest theoretical methods, to compute current driver power for all four options under the same set of circumstances. First, all drivers are required to generate steady state current in a reactor typical of low power operation of INTOR. In this mode (see Table I) the density is roughly half the reference INTOR value ${ }^{5}$ so the power and neutron wall load are roughly one quarter of the reference values. The second aim of the comparisons is to learn what degree of current 
Table I

Geometry and PI asma

\begin{tabular}{|c|c|c|c|c|}
\hline Parameter & Symbol & Units & \multicolumn{2}{|l|}{ Value } \\
\hline Major radius & $R_{0}$ & $m$ & \multicolumn{2}{|l|}{4.9} \\
\hline Aspect ratio & $A$ & & \multicolumn{2}{|l|}{4.2} \\
\hline Elongation & $\kappa$ & & \multicolumn{2}{|l|}{1.6} \\
\hline Triangu larity & $\delta$ & & \multicolumn{2}{|l|}{0.5} \\
\hline Vacuum field & $B_{0}$ & $\mathrm{~T}$ & \multicolumn{2}{|l|}{5.5} \\
\hline Axis safety factor & $q_{a}$ & & \multicolumn{2}{|l|}{$\sim 1.0$} \\
\hline Boundary safety factor & $q_{b}$ & & \multicolumn{2}{|l|}{$\sim 2.0$} \\
\hline Density profile & $n_{e}(\psi)$ & & \multicolumn{2}{|c|}{ "parabolic" } \\
\hline Temperature profile & $T_{e}(\psi)$ & & \multicolumn{2}{|c|}{ "parabolic" } \\
\hline Current density & j & & centrally $\mathrm{p}$ & eaked \\
\hline Average density & $\bar{n}_{e}$ & $10^{20} m^{-3}$ & \multicolumn{2}{|l|}{0.7} \\
\hline Average temperature & $\bar{T}_{e}$ & keV & 20 & steady \\
\hline Electric field & $E$ & $\mathrm{~V} / \mathrm{m}$ & 0 & state \\
\hline Toroidal current & lo & $M A$ & \multicolumn{2}{|l|}{8.0} \\
\hline Average density & $\bar{n}_{e}$ & $10^{20} m^{-3}$ & \multicolumn{2}{|l|}{0.04} \\
\hline Average temperature & $T_{e}$ & $\mathrm{keV}$ & 2 & reversed \\
\hline Electric tield & $E$ & $\mathrm{~V} / \mathrm{m}$ & \multicolumn{2}{|l|}{-0.01} \\
\hline Toroidal current & 1 & MA & \multicolumn{2}{|c|}{8.0 , net } \\
\hline
\end{tabular}


density profile control may be achieved with the four options. Flexiblitity in this aspect of current drive is valuable, as such a technique might be employed to improve the plasma operating conditlons (e.g., to eliminate sawteeth, reduce disruption frequency, or operate at higher beta). The third goal is to compute current drive requirements with a reversed emf $(E<0)$ at very low density (see Table 1). This situation is typical of noninductive, slow current rampup or of a hybrid burn cycle in which the transformer is periodically recharged. 6 In this situation the inductive and noninductive currents might be combined in a fashion which is not simply additive; a generalization of Ohm's Law may need to be used to infer the current drive requi rements.

\section{STEADY STATE}

\subsection{Flgure of Merlt and Sensitivity to Plasma Paraneters}

Most basic theories of current drive deal with infinite homogeneous plasma, while it is useful for INTOR and reactor designs to convert the local current drive efficlency to a global ratio of generated toroldal current, $1_{0}$, to absorbed plasma heating power, $P_{C D}$. In addition, the toroidal stacking effect leads to a dependence $1_{0} / P_{C D} \propto R_{0}^{-1}$, whereas the current carriers are generally slowed by collisions such that $1_{0} / P_{C D} \propto n_{e}^{-1}$. Hence, to lowest order, the ratio

$$
\gamma \equiv 1_{0} \bar{n}_{e} R_{0} / P_{C D}
$$

is expected to be roughly constant in tokamaks. This ratio, $Y$, is a figure of merit which must be maximized in order to minimize capital costs and circulating power for current drive. It is expected that profiles $n_{e}(\psi)$ and $T_{e}(\psi)$, where $\psi$ is the poloidal flux, will have some influence on the value of $\gamma$. Likewise, the calculations should ideally determine whether there are additional parameter dependences, such as $\bar{T}_{e}$ or $\bar{T}_{i}$ (electron and ion temperatures), which may determine the value of $\gamma$. For the wave drivers the plasma has $Z_{\text {eff }}=1.5$, while the NB driver benefits from larger disparity between the beam charge and $z_{\text {eff }}$, so $z_{\text {eff }}=2.0$ is selected.

The degree of sophistication varies among the calculational methods used 
for the drivers. For example, the NB calculation assumes concentric elliptical flux surfaces and the generated current density profile is not consistent with that assoclated with the flux surfaces. In contrast, the wave drivers all create current density which is in two dimensional toroldal MHD equilibrium; the flux surfaces display the Shatranov shlft and have shapes determined by the Grad-Shafranov pressure balance equation. 7 On the other hand, the wave drive models have not yet included alpha particle damping, which potentially could be a limiting factor. Thus, the values of $\gamma$ determined by these calculations should be vlewed as preliminary and subject to refinement in the future.

\subsection{LLH Current Drive}

Historically, the greatest concern with the LH wave is its poor penetration into high density and temperature plasmas. Thus, the achievement of centrally peaked current profiles (Table 1 ) is not predicted for this option. A number of calculations of $\gamma$ from earlier sources, complled in Table I1, will be briefly discussed before analyzing INTOR.

The first three entries in Table 11 , from Ref. 8, demonstrate that $\gamma$ drops for a reastor as greater penetration is achleved. This is because the current is generated where the local electron density is progressively higher, whereas the average value of $\bar{n}_{e}$ is constant in the sequence tabulated. In Ref. 8 it was not possible to get current drive near the magnetic axis.

The next seven entries in Table 11 , from Ref. 9, use an improved calculation of LHCD in which the clrcular flux surfaces are in MHD equilibrium (at $A$ $\rightarrow \infty)$ with the pressure and current density. This set of calculations explic$i t$ ly shows that deep wave penetration is possible at low $\bar{n}_{e}$ and $\bar{T}_{e}$, for example, during the initlal phase of current ramp up. At low temperature $Y$ is small ( $\gamma=0.13$ at $\bar{T}_{e}=1.8 \mathrm{keV}$ ) but increasing with $\overline{\mathrm{T}}_{e}$. At high $\overline{\mathrm{T}}_{\mathrm{e}}$ the value of $r$ appears attractive, but of course the equilibrium has a hollow current density. It is noteworthy that $\gamma\left(\bar{T}_{e}=1.8 \mathrm{keV}\right)=0.13$ is in accord with experimental values at that temperature, as evidenced by Fig. 1 . One unsettled issue concerns the spectral gap. The sfactrun for $\overline{\mathrm{T}}_{e}=1.8 \mathrm{keV}$ in Table 11 is centered at $\bar{n}_{1}=3.1$ but extends from $n_{1}=1.9$ to $n_{1}=4.3$, whicil pulis electrons out from the themal distribution towards the high energy tail. The 
Teble II

Previous Y Calculations for LHCD

\begin{tabular}{|c|c|c|c|c|c|c|c|c|}
\hline $\begin{array}{l}R_{0} \\
(m)\end{array}$ & $\begin{array}{c}\overline{n_{e}} \\
\left(10^{20} m^{-3}\right)\end{array}$ & $\begin{array}{l}\text { 10 } \\
\text { (MA) }\end{array}$ & $\begin{array}{l}P_{C D} \\
(M W)\end{array}$ & $\gamma$ & $\begin{array}{c}\text { Penetration } \\
q\end{array}$ & $\begin{array}{l}\bar{T}_{e} \\
(k e v)\end{array}$ & $\bar{n}_{n}$ & Ref. \\
\hline 7.0 & 0.83 & 9.8 & 59 & 0.97 & 28 & 18 & 1.42 & 8 \\
\hline 7.0 & 0.83 & 9.8 & 76 & 0.75 & 32 & 18 & 1.48 & 8 \\
\hline 7.0 & 0.83 & 9.8 & 110 & 0.52 & 41 & 18 & 1.54 & 8 \\
\hline 7.0 & 0.11 & 2.45 & 15 & 0.13 & 85 & 1.8 & 3.1 & 9 \\
\hline 7.0 & 0.11 & 18.5 & 40 & 0.35 & 80 & 5.5 & 2.1 & 9 \\
\hline 7.0 & 0.11 & 24.1 & 40 & 0.46 & $\sim 50$ & 6.3 & 2.1 & 9 \\
\hline 7.0 & 0.39 & 14.7 & 90 & 0.45 & 30 & 13.1 & 1.6 & 9 \\
\hline 7.0 & 0.77 & 10.0 & 90 & 0.60 & $\sim 25$ & 15.6 & 1.6 & 9 \\
\hline 7.0 & 0.88 & 14.4 & 90 & 0.99 & 20 & 17.7 & 1.6 & 9 \\
\hline 7.0 & 1.18 & 10.1 & 90 & 0.92 & 14 & 17.3 & 1.6 & 9 \\
\hline 3.0 & 1.13 & 4.16 & 45 & 0.31 & 35 & 8.0 & 1.5 & 10 \\
\hline 3.0 & 1.13 & 4.06 & 45 & 0.37 & $\sim 30$ & 10.5 & 1.5 & 10 \\
\hline 3.0 & 1.13 & 5.16 & 45 & 0.39 & 23 & 15.5 & 1.5 & 10 \\
\hline 3.0 & 1.13 & 2.86 & 45 & 0.22 & 37 & 7.4 & 1.5 & 10 \\
\hline
\end{tabular}

1. Ref. 8 model: concentric clrcular flux surfaces, uniform flux; $f=1.5$ $\mathrm{GHz}, \mathrm{Z}_{\text {eff }}=1.7$.

2. Ref. 9 model: MHD equilibrium $(A+\infty)$ circular flux surfaces; uniform flux; $f=1.7 \mathrm{GHz}, z_{\text {eff }}=3.4$.

3. Ref. 10 model: shifted (Shafranov) analytic flux surfaces; ray tracing, bottom launch; $f=4.5 \mathrm{GHz} ; T_{1}=T_{e}$. 


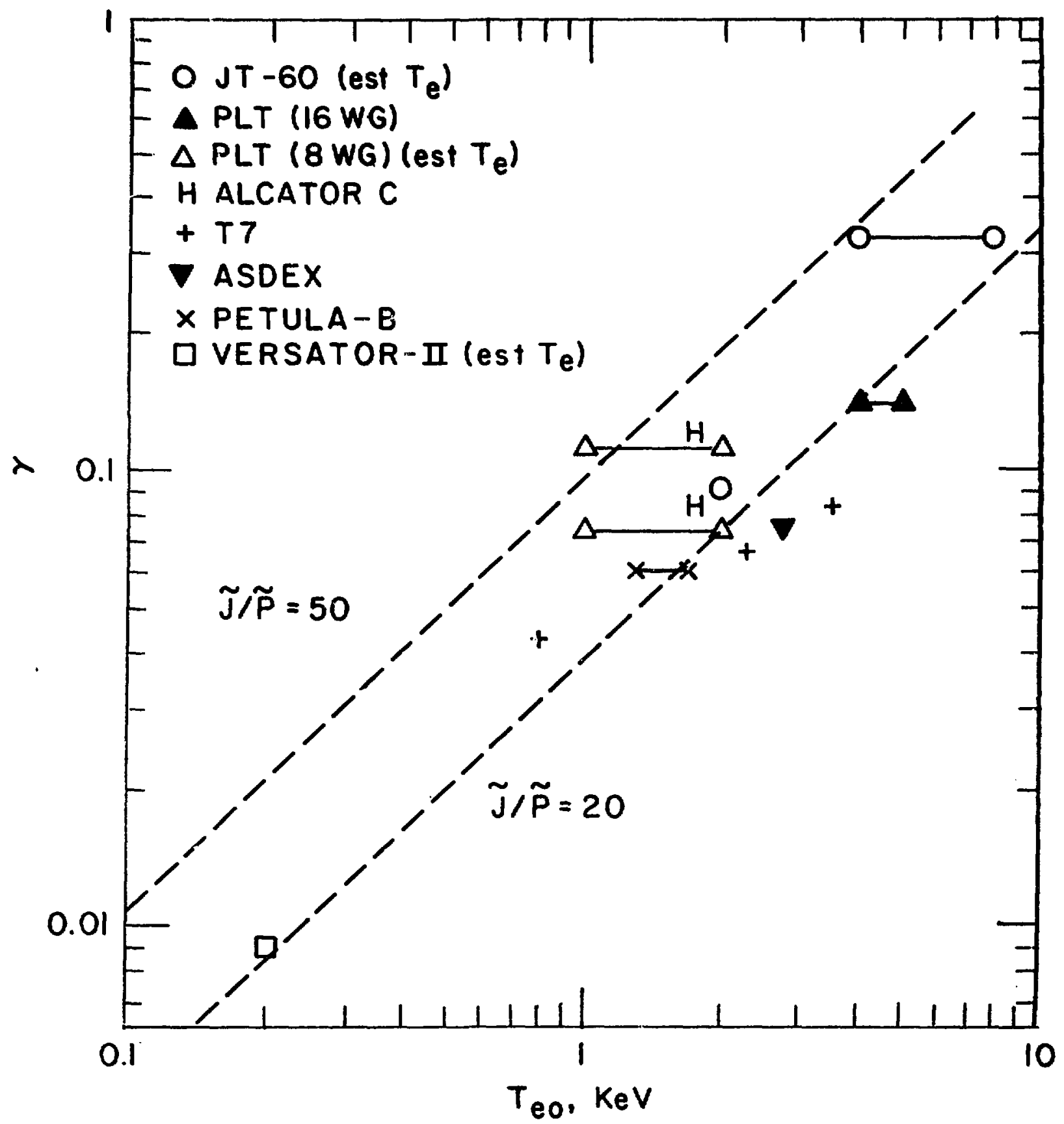

Figure 1: Experimental $\gamma=\bar{n}_{e} \mid R_{o} / P i n j\left(10^{20} m^{-3}-M A-m / M W\right)$ for steady state lower hybrid current drive with $v_{\text {loop }}=0$ and $I=0$. Pinj is total injected power, ignoring directivity and accessibility. Dashed lines are theoretical values, assuming total absorption of injected power. 
experiments generally operate with $\bar{n}_{\|}=2.0$ and with $\Delta n_{n}$ often considered too narrow to interact with thermal electrons.

The final antries in Table 11 , performed for an ETR candidate, 10 also show a decrease in penetration as $\bar{T}_{e}$ increases. The final case, at $\bar{T}_{e}=7.4$ keV, achieves deeper penetration by using a narrower $T_{e}$ profile, however $\gamma$ suffers because current drive is occurring at a higher local $\mathrm{n}_{e}$.

The trends in Table 11 were confirmed with our own calculations. We improve on the previous models by computing LH generated current in MHD equilibrium (at arbitrary A .7 Ray tracing is periormed with launch at the outboard midplane into the steady state INTOR described in Table 1, with $Z_{\text {eff }}$ $=$ 1.5. The profiles are $T_{e}(\tilde{\psi})=T_{e o} \tilde{\psi}^{\alpha} T$ and $n_{e}(\psi)=n_{e o} \tilde{\psi}^{\alpha} n$ where $\tilde{\psi} \equiv\left(\psi_{b}-\right.$ $\psi) /\left(\psi_{b}-\psi_{a}\right)$ and $a, b$ denote values at the axis and boundary, respectively. He choose $\alpha_{T}=0.7$ and $\alpha_{n}=0.7$, so the pressure protlle is $p(\psi)=p_{0} \tilde{\psi}^{\alpha}$, with $\alpha$ $=\alpha_{T}+\alpha_{n}=1.4$.

The benchmark case requires $T_{e}=20 \mathrm{keV}$, so $T_{e o}=35 \mathrm{keV}$. At these very high temperatures, deep penetration cannot be achieved. Figure $2 a$ illustrates the ray path for a mode with $f=5.0 \mathrm{GHz}$ and $n_{n}=1.6$. The radial group velocity is slow and the wave propagates nearly tangentially to the flux surfaces. As shown in Fig. $2 b$, electron Landau damping peaks near $n_{e} / n_{e o} \simeq$ 0.44 and the wave is totally absorbed without further propagation. While

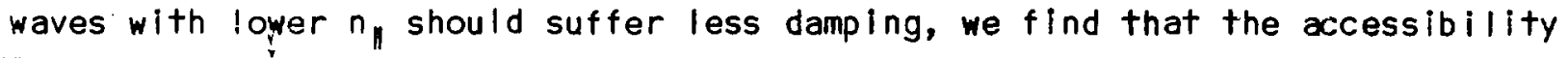
iimit prevents propagation at lower $n_{1}$.

Changing the $\mathrm{T}_{e}(\psi)$ profile has an effect on depth of penetration, but, if $T_{e}$ is held constant, there is found to be no significant improvement.

The basic Landau damping process is very weak except where $w \equiv$ $\omega / k_{\|} v_{e} \simeq 3$. At the temperature where this criterion is satisfied, the damping and current generation are maximized. The most successful strategy to achieve deeper LH wave penetration is to reduce $T_{e}$. Figure $3 a$ shows the ray path at $T_{e}=10 \mathrm{kev}$ for $f=5.0 \mathrm{GHz}$ and $n_{n}=1.8$. In this case $n_{e} / n_{e o}=0.63$ at furthest penetration, whic. is an improvement.

At $T_{e}=5 \mathrm{keV}$ the results are better, although full penetration is still not achieved. If ion and electron temperatures are sufficiently disparate, it 

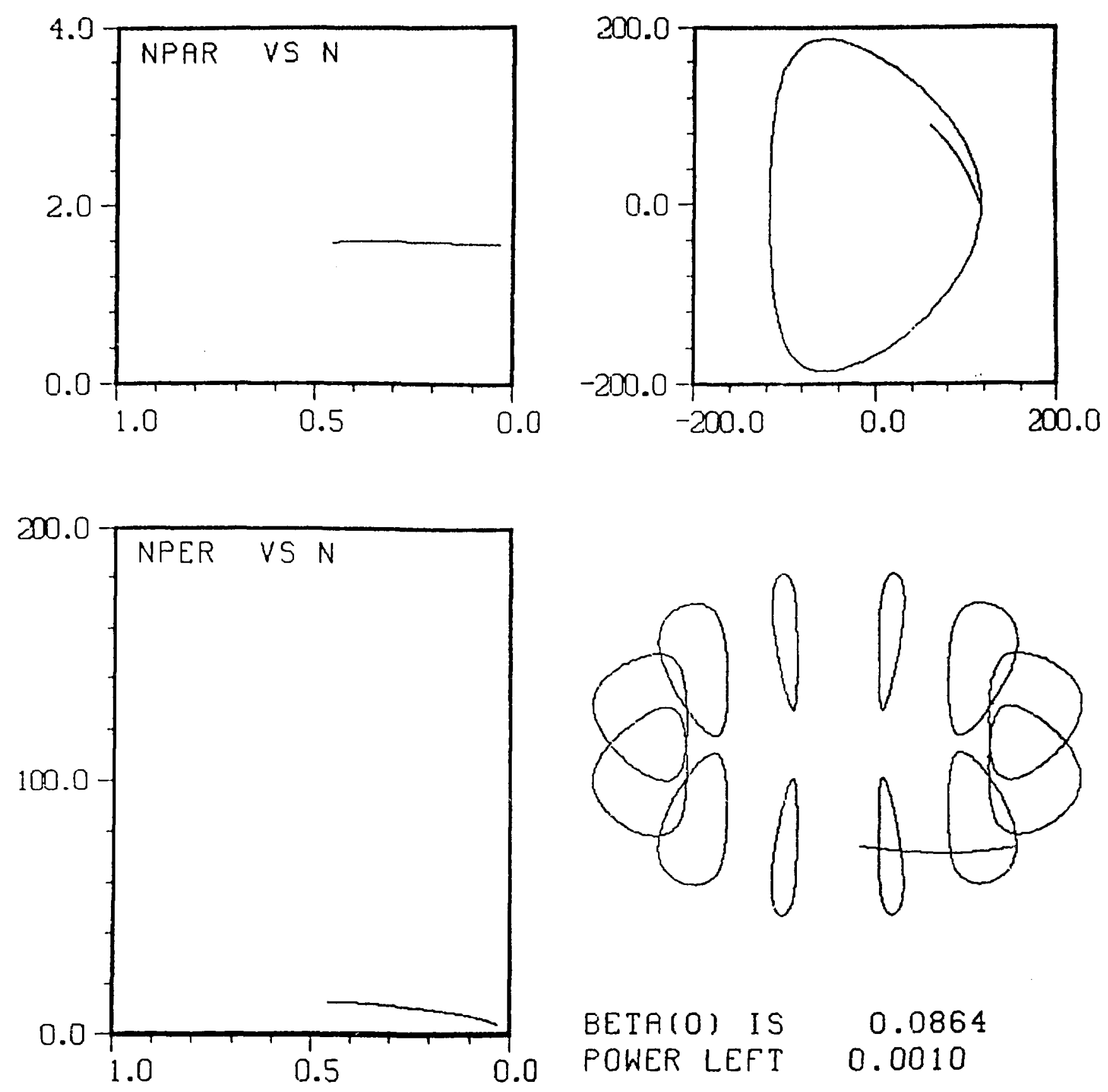

BETA(O) IS 0.0864 POWER LEFT 0.0010

Figure 2a: Ray path for LH wave at $f=5.0 \mathrm{GHz}, n_{n}=1.6$, in a plasma with $T_{e}=20 \mathrm{keV}, T_{\text {eo }}=35 \mathrm{keV}, n_{\text {eo }}=1.23 \times 10^{20} \mathrm{~m}^{-3}, T_{10}=18$ $\mathrm{keV}, \mathrm{B}_{0}=5.5 \mathrm{~T}$, and $\mathrm{R}_{0}=4.9 \mathrm{~m}$. 

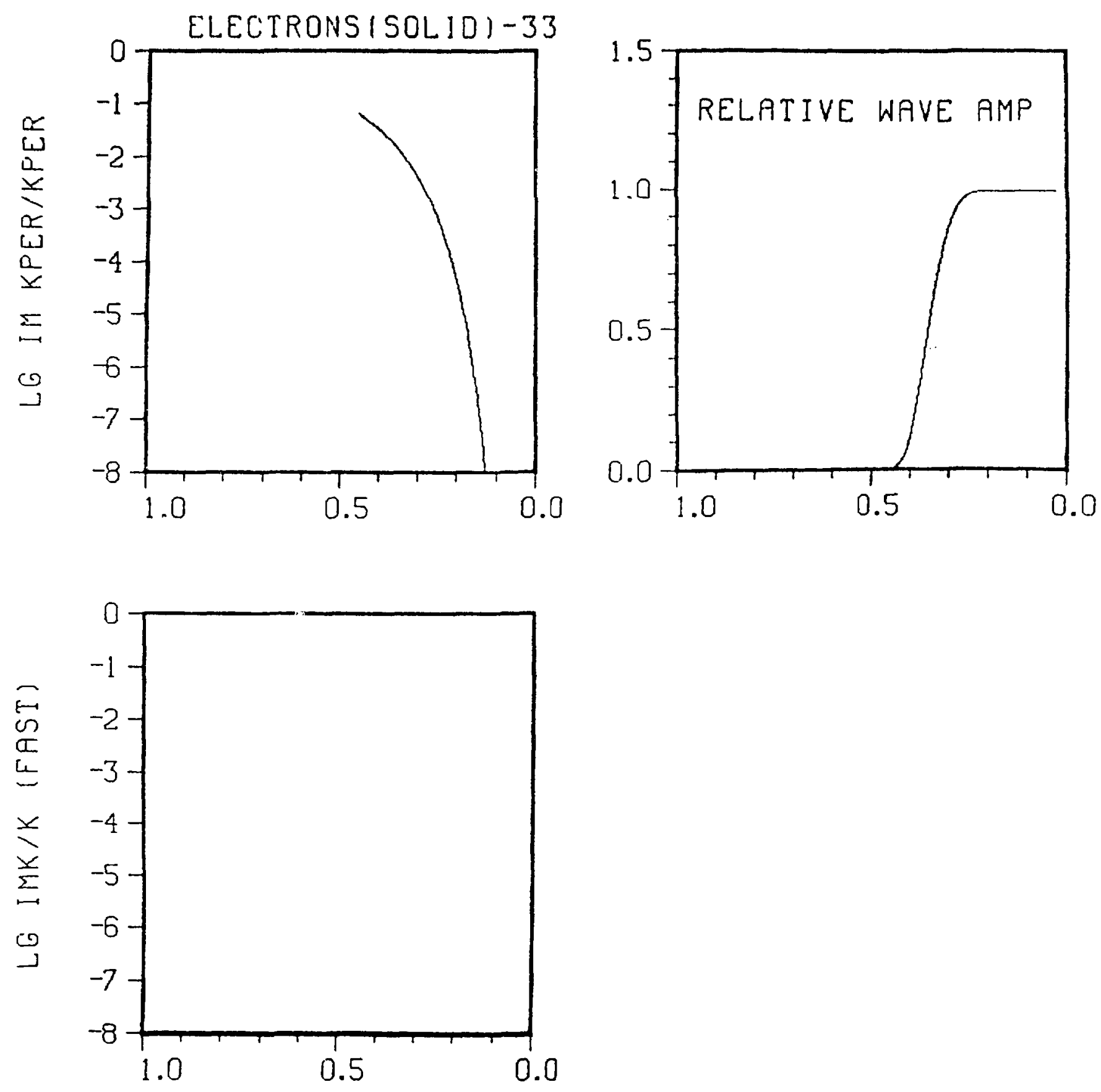

Figure 2b: Damping of wave in Fig. $2 a$. 

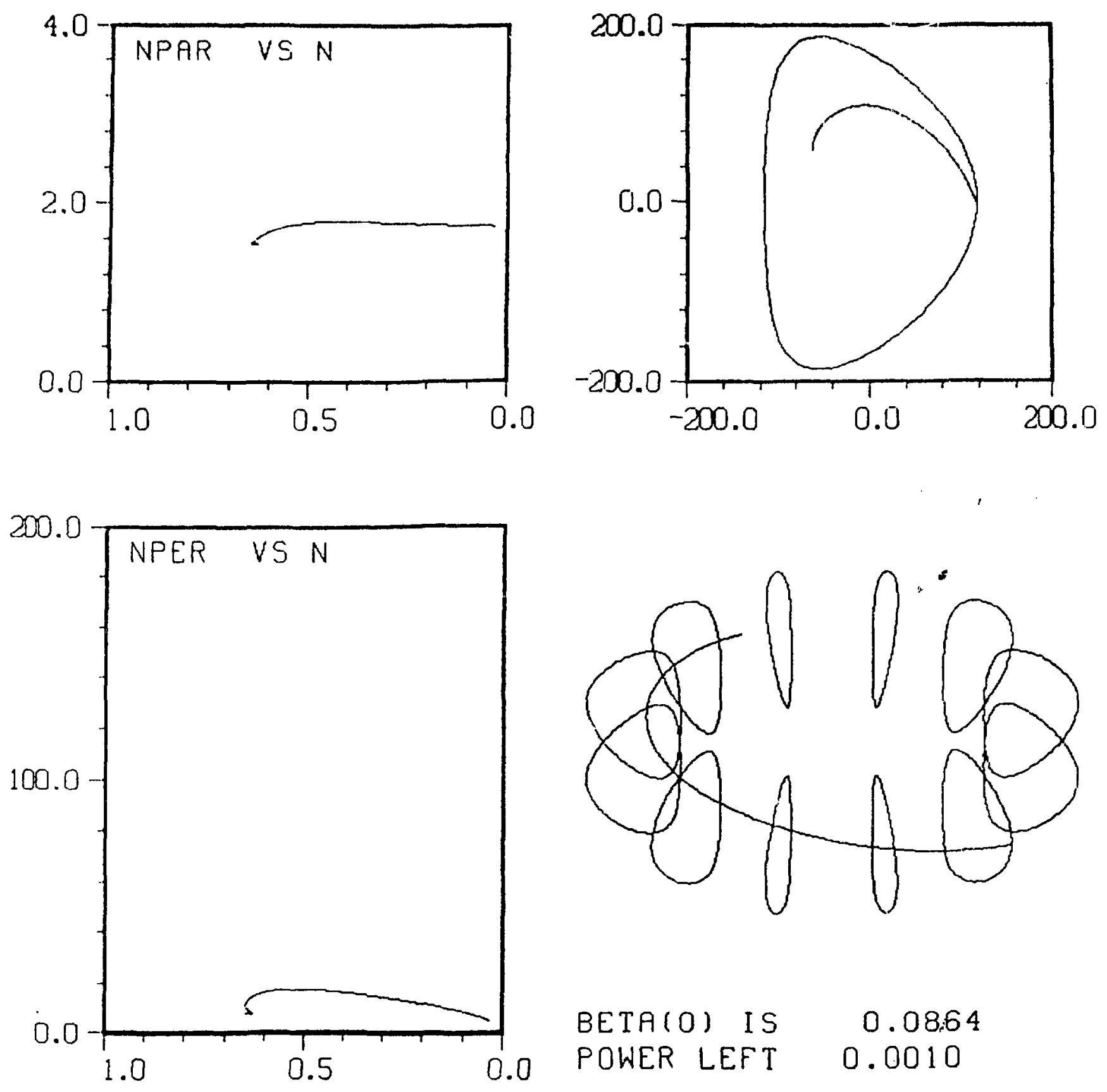

Figure 3a: Same as Fig. 2 except $T_{e}=10 \mathrm{keV}, T_{\text {eo }}=18 \mathrm{keV}, T_{10}=35 \mathrm{keV}$, and $n_{k}=1.8$. Abscissa for $n_{1}$ and $n_{\perp}$ plots is $n_{e} / n_{e o}$. 

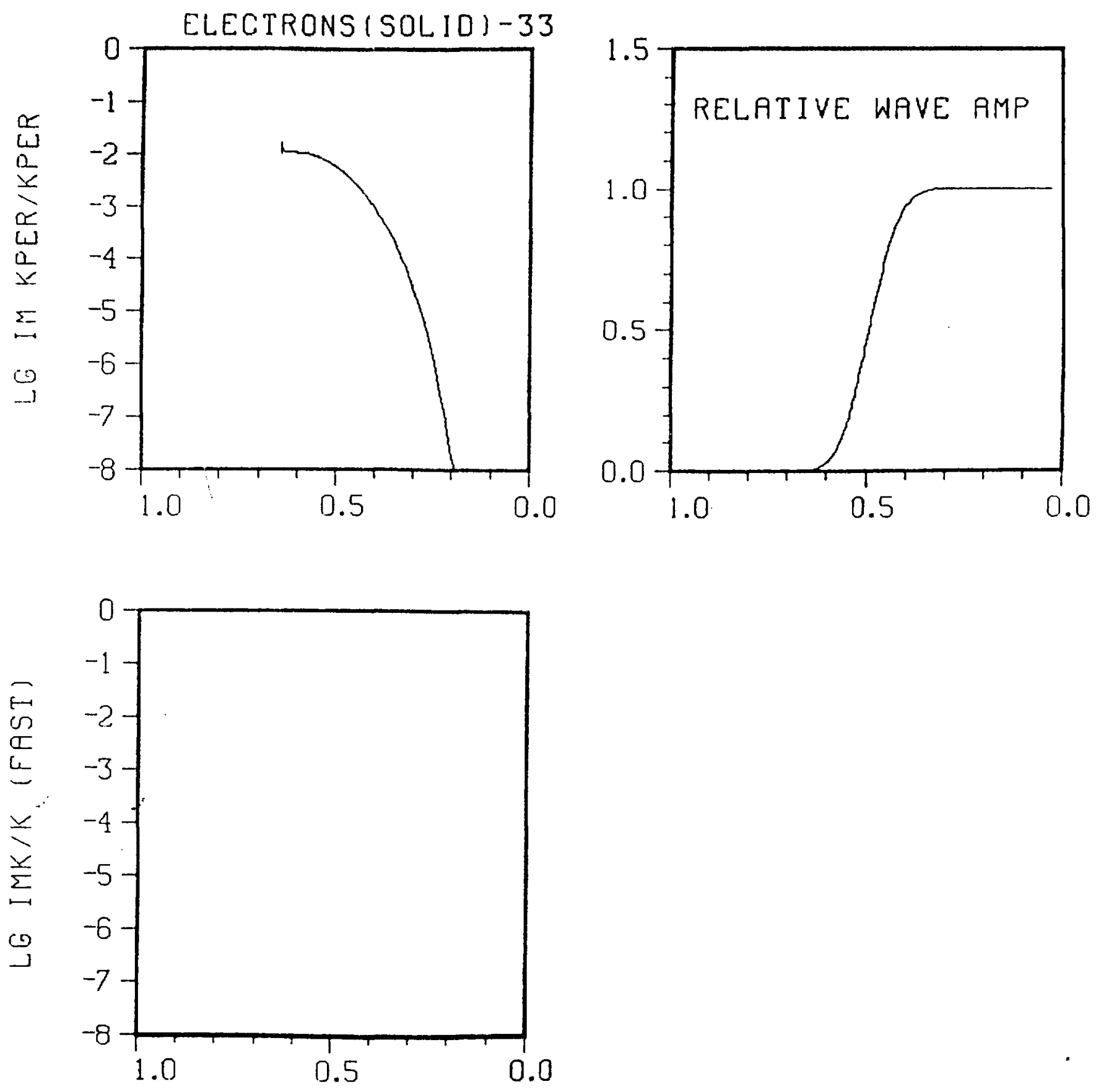

Figure 3b: Wave penetrates further without damping as $T_{e}$ is lowered. 
may be possible to stlll produce significant fusion power even with such a low $T_{e}$ value. The first equilibrium we studied had $T_{e o}=8.8 \mathrm{keV}, \overline{\mathrm{T}}_{e} \simeq 5$ $\mathrm{keV}, n_{\text {eo }}=1.23 \times 10^{20} \mathrm{~m}^{-3}$, and $T_{i 0}=26.4 \mathrm{keV}$, with $\alpha_{T}=\alpha_{n}=0.7$ Figure 4 shows the ray trajectory for a typical mode with $f=10 \mathrm{GHz}$ and $n_{\|}=1.9$. The wave damping and current drive maximize at $n_{e} / n_{e o} \simeq 0.80$ for this wave. By selecting a spectrum of modes, it is possible to generate current at a continuum of minor radii. This was done using the following modes:

\section{Frequency (GHz)}

5.0

7.0

7.0

10.0

10.0

10.0

10.0

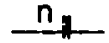

4.0

3.2

2.7

2.4

2.1

1.9

1.8

\section{Edge Power (MW)}

1.0

4.0

7.4

5.2

8.7

40.6

19.7

The resulting converged equilibrium is shown in Fig. 5a. The function $G \equiv$ $\langle j|| B\rangle /\left\langle B^{2}\right\rangle$ is displayed vs. $\tilde{\psi}$. As $n \|$ decreases, the modes are damped successively closer to the magnetic axis $(\tilde{\psi}=1)$. Lower $n_{\text {n }}$ results in reflection. If there were no spatial redistribution of current this would result in a hollow current profile with $j=0$ at the axis. In the calculation of Fig. 5a, we allowed the profile to spread by averaging the value of $G$ over

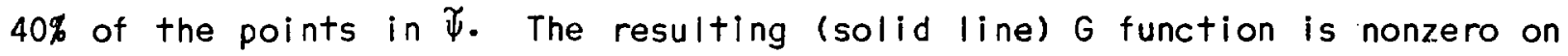
the axis. The corresponding $j_{+}(R, z=0)$ is also nonzero on the axis although the profile is hollow. The safety factor, as shown, is nonmonotone in $q(\psi)$ and is likely to be MHD unstable. The total power, $P_{C D}=86.7 \mathrm{MW}$, results in an equilibrium with $\beta_{+}=0.0506$ and $\mathrm{I}_{0}=8.2 \mathrm{MA}$. The actual average values obtained are $\bar{n}_{e}=0.73 \times 10^{20} \mathrm{~m}^{-3}$ and $T_{e}=5.2 \mathrm{keV}$, yielding $r$ $=0.338$.

The identical spectrum was applied and solved for equilibrium with averaging over $50 \%$ of the $\tilde{\psi}^{\prime}$ points. This resulted in $\beta_{+}=0.0452$ and $1_{0}=8.1$ MA with $\bar{n}_{e}=0.70 \times 10^{20} \mathrm{~m}^{-3}$ and $\overline{7}_{e}=5.0 \mathrm{keV}$. This case, with $\gamma=0.322$, has a less hollow $j$ profile.

Likewise, this spectrum was studied with $60 \%$ averaging. This is illustrated in Fig. $5 b$ where we find that the converged $j$ profile is broad but 

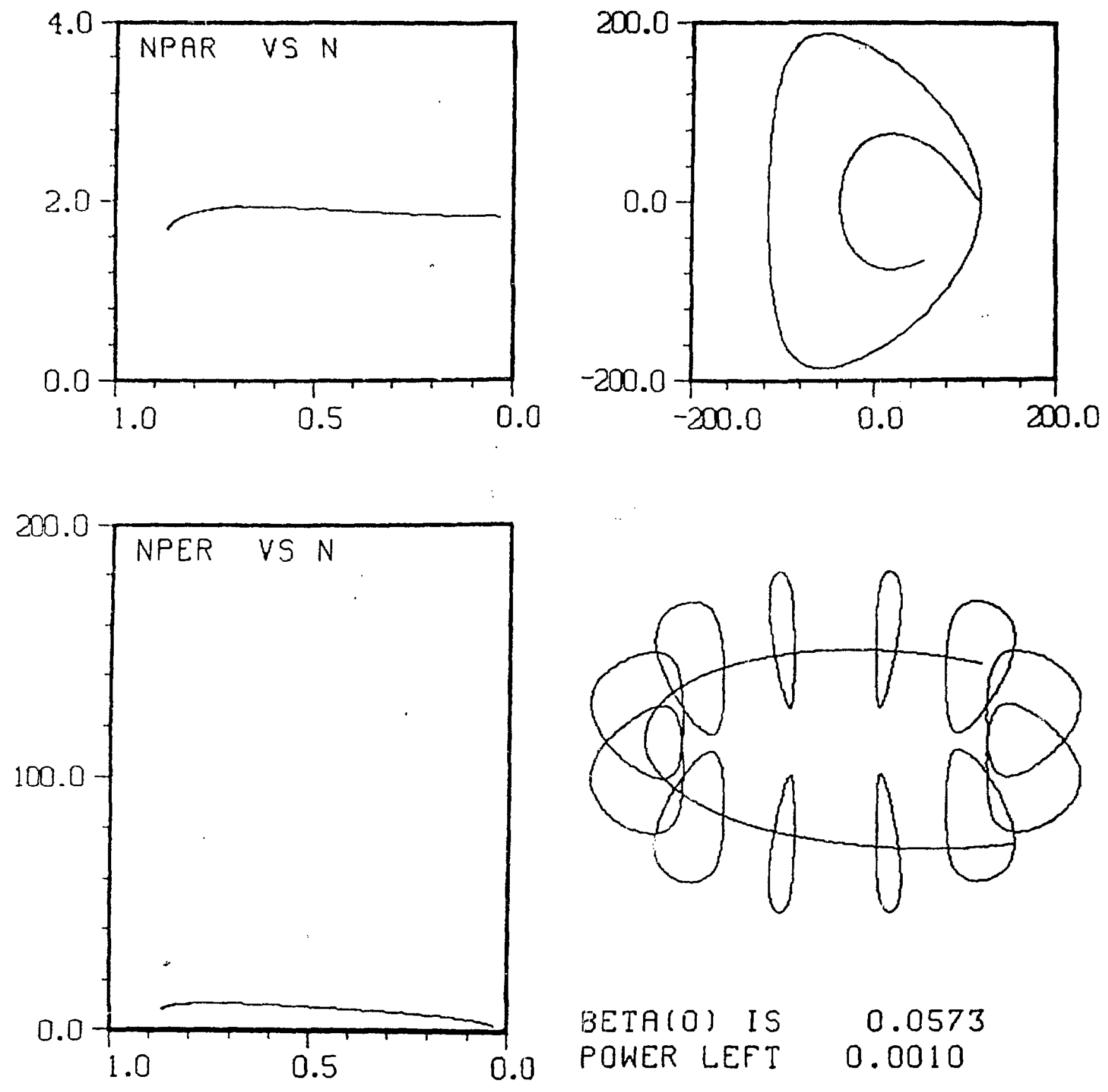

BETA(O) IS POWER LEFT

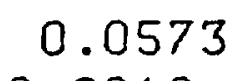

0.0010

Figure 4a: Ray path for LH wave at $f=10 \mathrm{GHz}, n_{n}=1.9$, in plasma with $\bar{T}_{e}=$ $5 \mathrm{keV}, T_{e o}=8.8 \mathrm{keV}, n_{e o}=1.23 \times 10^{20} \mathrm{~m}^{-3}, T_{i o}=26 \mathrm{keV}, B_{0}=$ $5.5 \mathrm{~T}$, and $R_{0}=4.9 \mathrm{~m}$. 

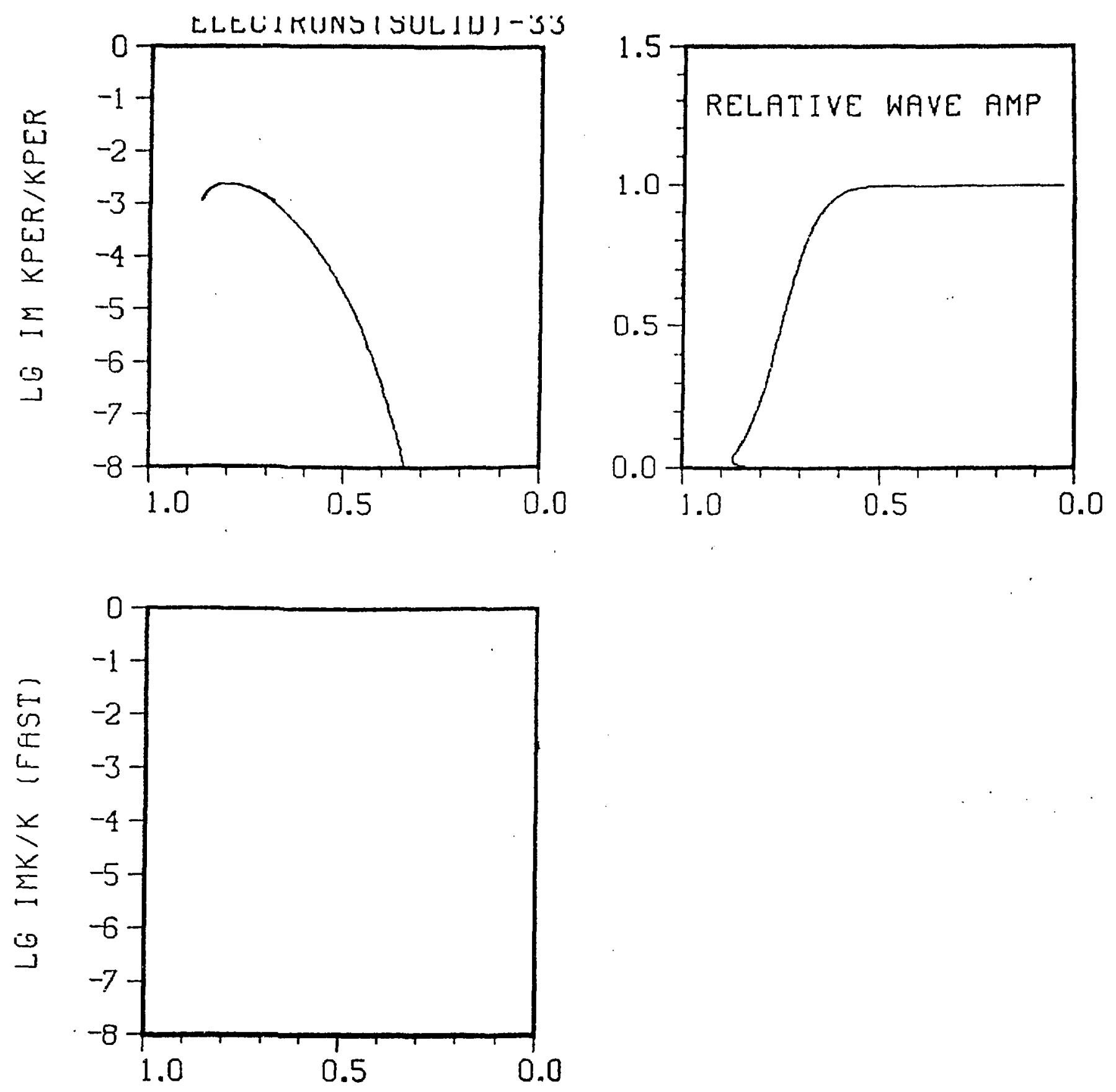

Figure 4b: Wave damps nearer the magnetlc axis as $T_{e}$ is reduced to $\sim 5 \mathrm{keV}$. 

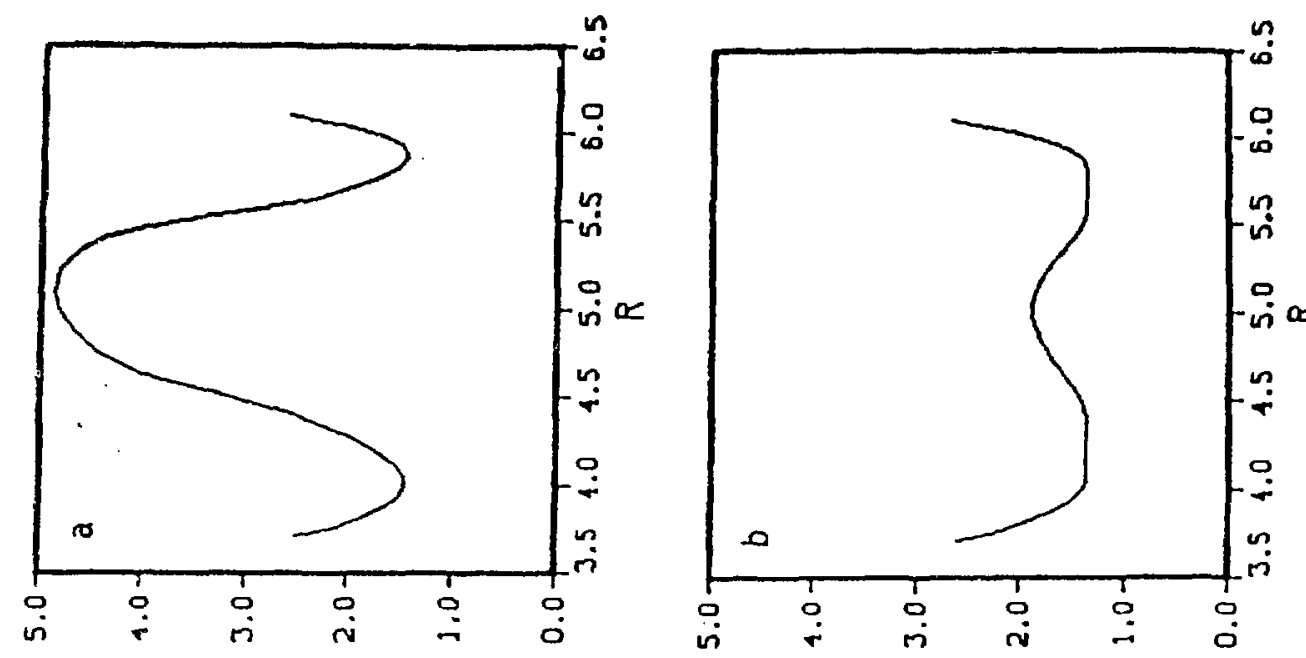

HOLOHS XI3JUS

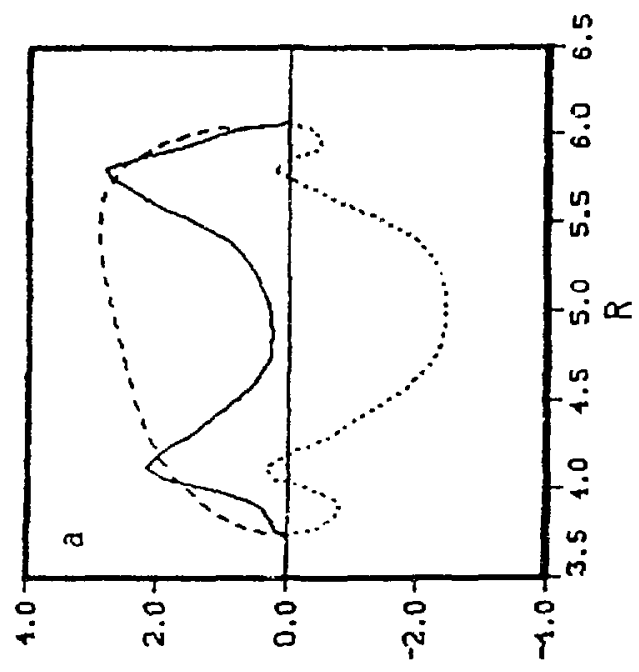

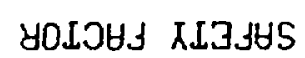
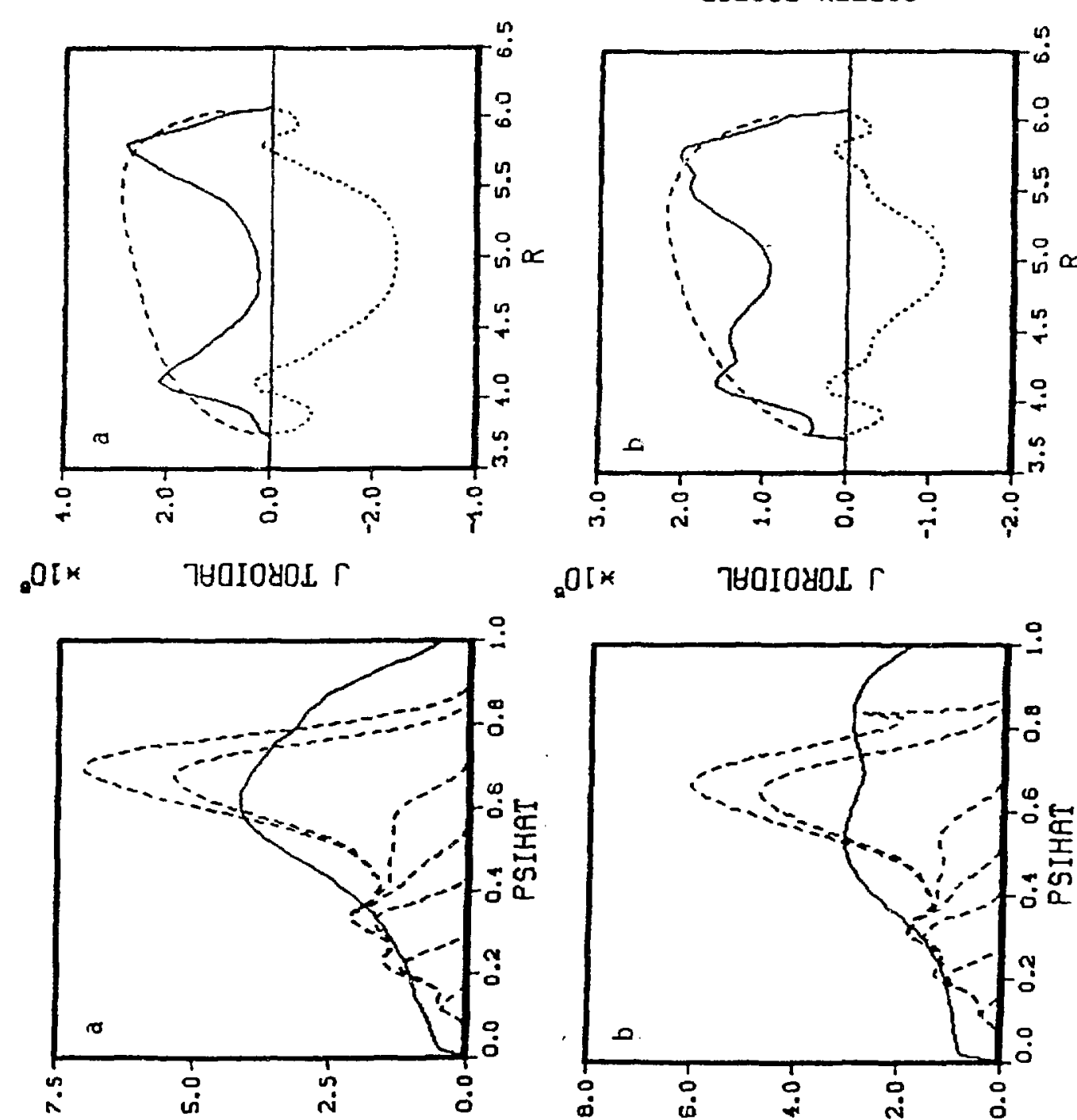

. $0[*$ 7HOIOYOL $r$

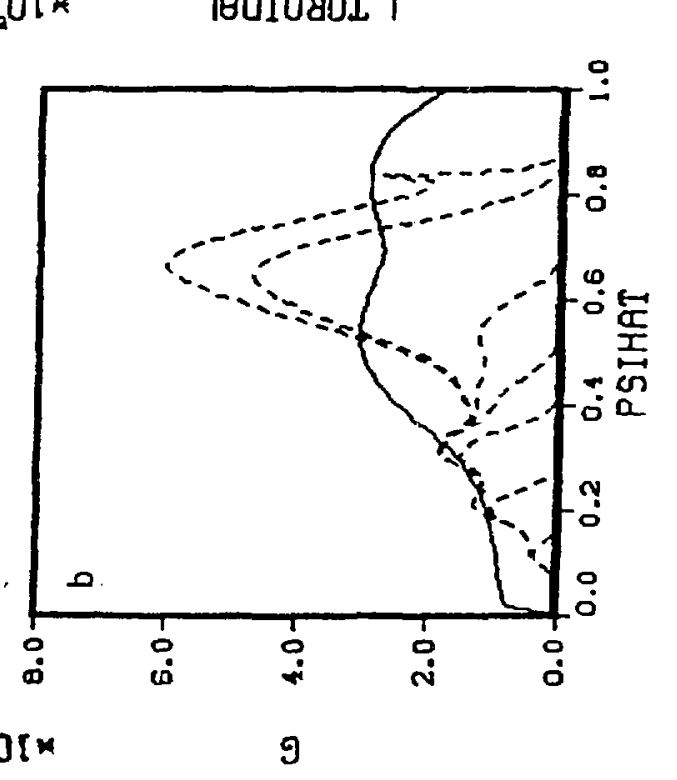

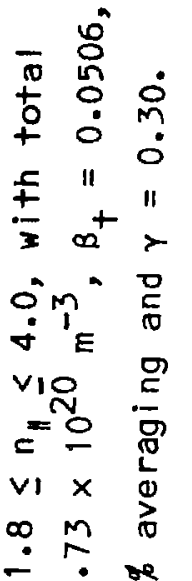

- 08

ㅇ.

VIIC $\frac{2 \pi}{x}$

Nㅗㅇ 호

- 鬲

$+\frac{1}{0}$

in 而

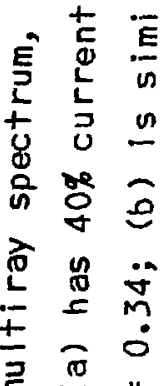

E -

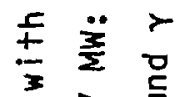

o $\infty$

$\therefore 11 \frac{x}{\Sigma}$

$\cong 8 \%$

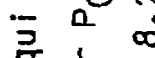

बै

工

$\ddot{n}$
$\frac{0}{3}$
$\frac{\sigma}{4}$

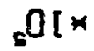

$\int_{0} 01 x$ 
still slightly hollow. Now $q(\psi)$ appears better behaved, although this equilibrium has not been analyzed for stablilty. This equilibrium has $B_{+}=$ 0.0409 and $I_{0}=7.9 \mathrm{MA}$ with $\bar{n}_{e}=0.68 \times 10^{20} \mathrm{~m}^{-3}$ and $T_{e}=4.8 \mathrm{keV}$, resulting in $\gamma=0.304$.

We can summarize the LH studies as follows

1. For the benchmark case, the LH waves do not penetrate far into the plasma, and centrally peaked current profiles are not predicted to occur. This conclusion is shared by previous investigators.8-11 our study assumed the poloidal mode number, $k_{\theta}$, is zero, but this is a reasonable choice as only small benefits to penetration should occur at nonzero values. 11

2. Lowering $\boldsymbol{T}_{e}$ to $\sim 5 \mathrm{keV}$ allows $\mathrm{LH}$ wave propagation and damping near the magnetic axis, and the corresponding value $\gamma=0.30$ is in rough agreement with the expectations from earlier studies isee Table 11). This value falls on a roughly linear extrapolation of the experimental values of $\gamma$ vs. $T_{e}$, seen in Fig. 1. Note, in particular $\gamma=0.14$ at $T_{\text {eo }} \cong 4 \mathrm{keV}$, as reported for PLT. 12

3. Radial transport of current density will be required, even at $\mathrm{T}_{\mathrm{e}} \simeq 5$ keV, to fill in the current density near the magnetic axis and avoid large $q_{a}$ peaking.

4. High frequencies $(\gtrsim 10 \mathrm{GHz}$ ) may be needed, and the design of such waveguide arrays may be challenging in a reactor envi ronment.

\subsection{HFFW Current Drive}

The superior penetration of the HFFW was noted in Ref. 11, where propagation near to the axis was found in the calculations. Detailed current drive calculations were reported in Refs. 7 and 13 (see Table 111). Reference 7 confirmed that centrally peaked $j$ is possible with the HFFW at reactorrelevant densities and temperatures. The values of $\gamma, \sim 0.2$ in Table 111 , do not display a clear dependence on $T_{e}$, and this is apparently due to the difficulty of achieving realistic $j$ profiles with a single mode. 
Table III

Previous $y$ Calculations for HFFW

\begin{tabular}{|c|c|c|c|c|c|c|c|c|}
\hline $\begin{array}{l}R_{0} \\
(m)\end{array}$ & $\begin{array}{c}\bar{n}_{e} \\
\left(10^{20} m^{-3}\right)\end{array}$ & $\begin{array}{l}\text { Io } \\
\text { (MA) }\end{array}$ & $\begin{array}{l}P_{C D} \\
(M W)\end{array}$ & $\gamma$ & $\begin{array}{c}\text { Penetration } \\
\varnothing\end{array}$ & $\begin{array}{l}\overline{\mathrm{T}}_{\mathrm{e}} \\
(\mathrm{keV})\end{array}$ & $\bar{n}_{n}$ & Ref. \\
\hline 5.25 & 0.40 & 4.4 & 50 & 0.18 & 100 & 13 & 2.4 & 7 \\
\hline 5.25 & 0.70 & 2.5 & 50 & 0.18 & $\sim 80$ & 13 & 2.4 & 7 \\
\hline 5.25 & 1.00 & 2.5 & 50 & 0.26 & $<80$ & 13 & 2.4 & 7 \\
\hline 5.25 & 1.00 & 1.4 & 50 & 0.18 & $<80$ & 8.9 & 2.4 & 7 \\
\hline 3.00 & 0.70 & 3. & 30 & 0.2 & $\sim 50$ & 4.0 & 1.35 & 13 \\
\hline
\end{tabular}

1. Ref. 7 model: $M H D$ equilibrium $(A=6, k=1.6, d=0.5)$, single ray; $f=$ $0.4 \mathrm{GHz}, \mathrm{Z}_{\text {eff }}=1.0$.

2. Ref. 13 model: concentric circular flux surfaces; spectral modification by edge density fluctuations; single ray; $f=1.5 \mathrm{GHz}$. 
In order to achieve centrally peaked currents without anomalous current diffusion and rearrangment it. is necessary to use a wide spectral band to deliver $r f$ power and current density throughout the plasma region. For this reason the computer code described in Ret. 7 was modified to include multiple waves in the spectrum. The HFFW with its large radial group velocity provides the bulk of the current throughout the plasma; about $1 \%$ of the power is transmitted as LH waves, which provide a small amount of current density near the plasma boundary, in order to properly adjust $q_{b}$ to the desired value.

We will briefly describe some details of the present code which represent improvements over Ref. 7. As usual the local ratio of current density to rf heating power density is

$$
j_{n} / P_{r f}=\frac{19.2 \times 10^{18}}{\ell n \Lambda} \frac{T_{e}}{n_{e}} \jmath / \tilde{P}_{r f},
$$

with $T_{e}$ in keV and other units MKs. However, the form of $Y / P_{r f}$ is a fit to various calculations ${ }^{14-15}$ which include the sundry parameter influences of interest to our problem:

$$
\tilde{j} / \tilde{P}_{r f}=\frac{8 w^{2}}{5+Z}+2+\frac{12(6+Z)}{(5+Z)(3+Z)}+\frac{2 D}{Z w} \text {, }
$$

where $w=w / k_{n} v_{e}, v_{e}=\sqrt{T_{e} / m_{e}}$, and $Z \equiv Z_{e f f}$. At low phase speeds inot of interest to the HFFW) there is a large increase of $\mathcal{J} / \mathrm{F}_{\mathrm{rf}}$ if TTMP is significant:

$$
D=\left\{\begin{array}{l}
3.76, \text { Landau damping. } \\
8.09, \text { TTMP }
\end{array}\right.
$$

For conservatism we ignore transit time magnetic pumping (TTMP), setting $D=3.76$ in all our calculations, although it is posslble that our results for $\gamma$ could increase by $\sim 20 \%$ if TTMP were indeed strong. In Fig. 6 our expression for $\tilde{J} / \widetilde{P}_{r f}$ is compared with other evaluations in different limits. The agreement is within $\sim 10 \%$ for the parameter range of interest. We find for linear damping that power absorption and current drlve maximize close to $w=2$, which, unfortunately, is near the minimum of the $J / \mathbb{P}_{r f}$ curves. since generally $T_{e_{0}} \leq 30 \mathrm{keV}$ we find relativistic effects are very minor.

The calculation starts with an initial equilibrium specitied with a glven 


\section{$Z=I$ NARROW SPECTRUM}

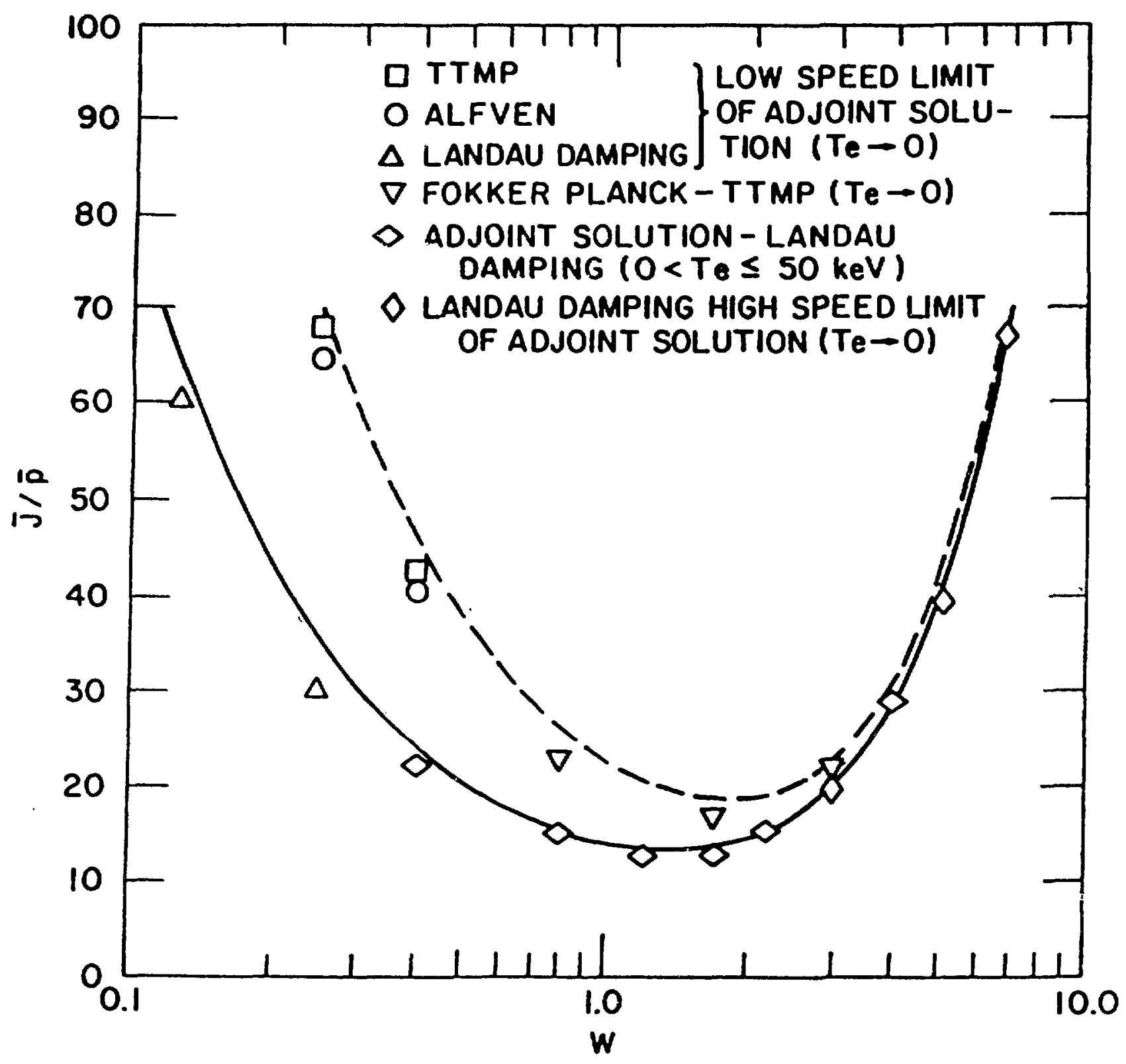

Figure ба: Normalized $\tilde{\jmath} / \tilde{p} r f$ : analytic expression (curves) compared to specific evaluations. Solid curve assumes Landau damping, dashed assumes TTMP. 


\section{$Z=2$ NARROW SPECTRUM}

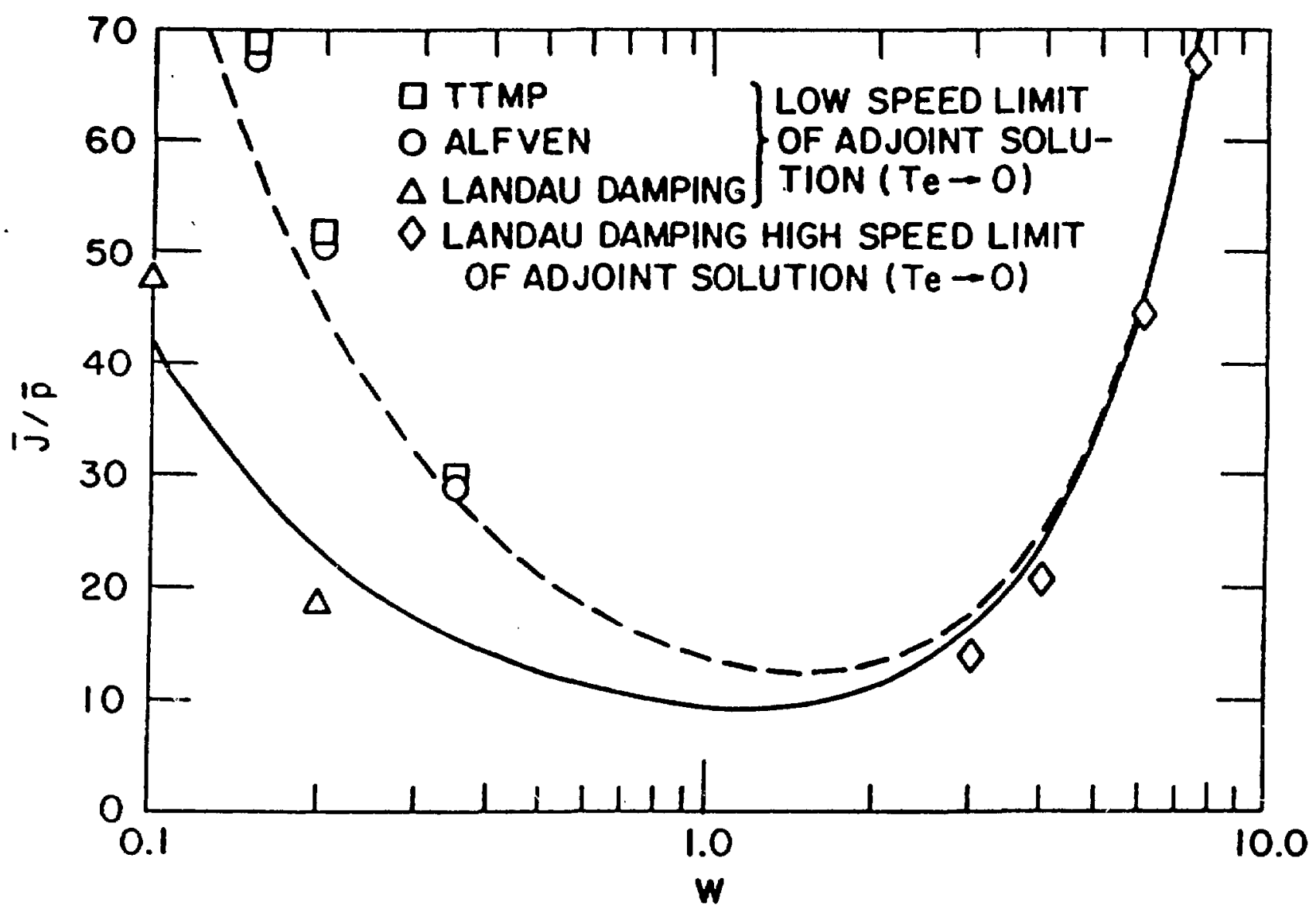

Figure 6b: Normalized $\tilde{J} / \tilde{p} r f$ with $Z_{e f}=2$. Abscissa is $w=\omega / k^{v}{ }_{e}$, where $v_{e}=\sqrt{T e^{/ m}}$. 
pressure, $p(\tilde{\psi})$, and model diamagnetism, $F(\tilde{\psi})$. Ray tracing for multiple waves is done on the $\psi(R, Z)$ surfaces (which satisfy the Grad-Shafranov equation), and the sum of all contributions yields the functional

$$
G(\psi)=\langle j, B\rangle /\left\langle B^{2}\right\rangle
$$

where s denotes the flux surface average. The actual diamagnetism associated with this power spectrum is found by solving

$$
\frac{1}{\mu} \frac{d F}{d \psi}+\frac{1}{\left\langle B^{2}\right\rangle} \frac{d p}{d \psi} F+\frac{G}{2 \pi}=0 \text {. }
$$

This value of $F(\psi)$ is introduced into the Grad-Shafranov equation and an updated solution for $\psi(R, Z)$ is found. Ray tracing is repeated on the updated $\psi$, and in this fashion the procedure is iterated until convergence. With a finite number of rays (10-30) and a $\tilde{\psi}$ grid divided into 100 boxes it is usually possible to have $1-2 \%$ convergence of the macroscop ic parameters ( $\beta_{+}$, $l_{o}, q_{a}, q_{b}$ ' after about five iterations. By properly adjusting the power spectrum we can achieve current profiles which appear to be stable (e.g., according to simple criteria such as the Troyon relationship). We then find $I_{O}, P_{C D}$, and $\gamma$ for a variety of $p l a s m a s$ of varying $\bar{T}_{e}, Z_{e f f}$, and other parameters. Our calculation of $\gamma$ is not unique, since various spectra can yield the same $L_{0}, B_{+}$, etc. with slightly differing details of $q(\psi)$ and current density. We estimate $\gamma$ values can vary by $\pm 5 \%$ due to the arbitrariness in selecting the spectrum.

Figure 7 is the INTOR benchmark case, which is characterized by $\bar{n}_{e}=0.7$ $\times 10^{20} \mathrm{~m}^{-3}, \bar{T}_{e}=20 \mathrm{keV}, R_{o}=4.9 \mathrm{~m}, B_{t}=0.0362, q_{a}=1.01, q_{b}=2.37$, and $Z_{\text {eff }}=1.5$. The spectrum includes both slow and fast waves: 

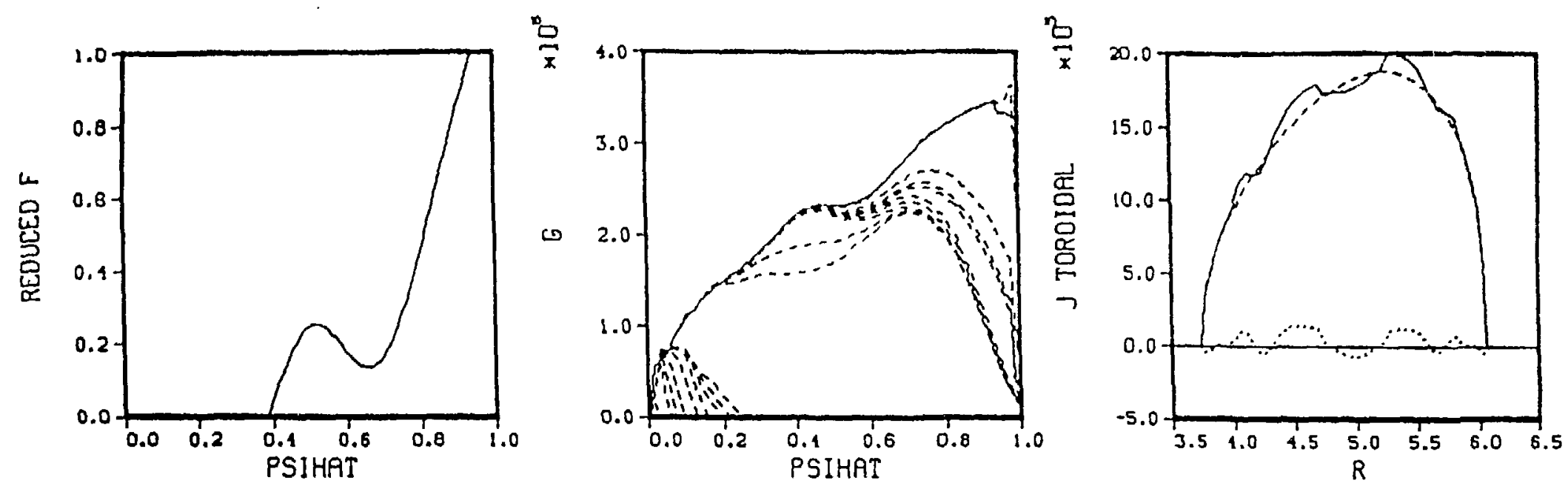

$\tilde{\omega}$
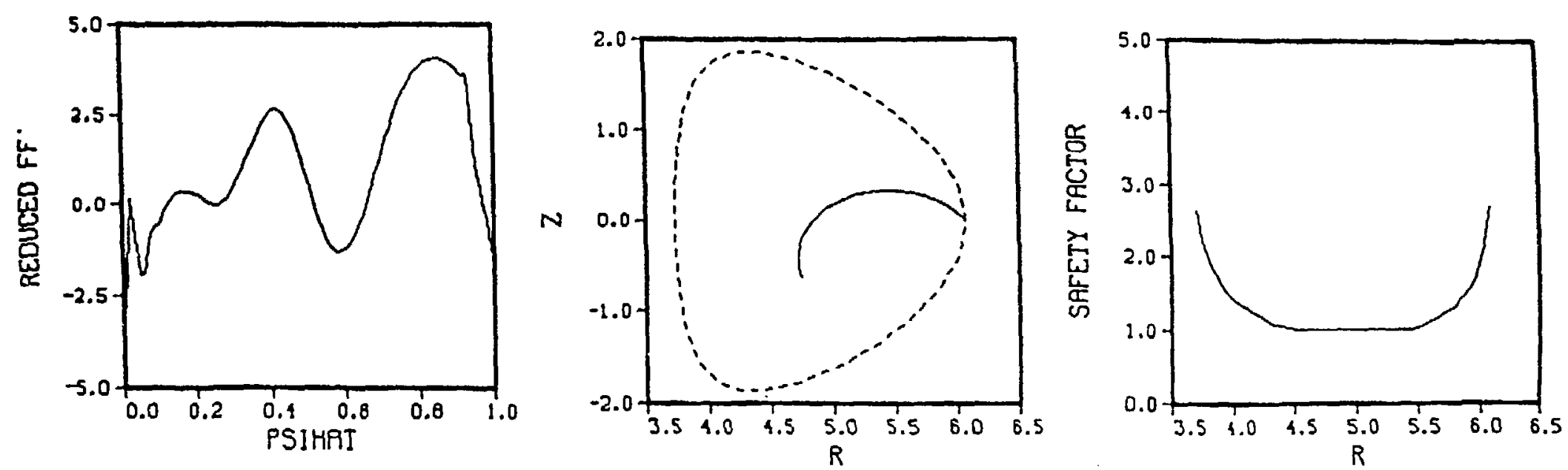

Figure 7: INTOR benchmark - equilibrium generated by HFFW with $\bar{n}_{e}=0.7 \times 10^{20} \mathrm{~m}^{-3}, 1_{0}=8.0$ $\mathrm{MA}, \mathrm{T}_{\mathrm{e}}=20 \mathrm{keV}, \mathrm{P}_{\mathrm{CD}}=67 \mathrm{MW}$, and $r=0.41$. Spectrum has $0.3 \leq \mathrm{f}(\mathrm{GHz}) \leq 1.0,1.6$ $\leq n_{1} \leq 2.8$; additional power $(\leq 1 \mathrm{MW})$ is provided as $3.0 \mathrm{GHz}$ LH waves. 


\begin{tabular}{|c|c|c|c|}
\hline Polarization & Frequency $(\mathrm{GHz})$ & $n_{1}$ & Edge Power (MW) \\
\hline LH & 3.0 & 4.4 & 0.05 \\
\hline LH & 3.0 & 3.4 & 0.27 \\
\hline LH & 3.0 & 2.9 & 0.21 \\
\hline LH & 3.0 & 2.6 & 0.25 \\
\hline LH & 3.0 & 2.3 & 0.39 \\
\hline LH & 3.0 & 2.1 & 0.31 \\
\hline LH & 3.0 & 1.9 & 0.25 \\
\hline LH & 3.0 & 1.8 & 0.15 \\
\hline LH & 3.0 & 1.7 & 0.22 \\
\hline$F W$ & 1.0 & 2.8 & 43.3 \\
\hline FW & 1.0 & 1.6 & 1.8 \\
\hline $\mathrm{FW}$ & 1.0 & 1.4 & 2.0 \\
\hline FW & 0.4 & 2.3 & 0.91 \\
\hline $\mathrm{FW}$ & 0.4 & 2.0 & 1.02 \\
\hline $\mathrm{FW}$ & 0.4 & 1.4 & 4.01 \\
\hline $\mathrm{FW}$ & 0.3 & 1.8 & 0.81 \\
\hline $\mathrm{FW}$ & 0.3 & 1.7 & 2.71 \\
\hline FW & 0.3 & 1.6 & 8.42 \\
\hline
\end{tabular}

The contributions of these modes to the cumulative value of $G(\tilde{\psi})$ are shown in Fig. 7. The waves in the figure are ordered as in the table (above), and they show progressively deeper penetration as the frequency is lowered, as previously found in Ref. 11. This calculation employed averaging over only $10 \%$ of the points, which is helpful in order to smooth the G function near the magnetic axis and to speed convergence. This spectrum employs a total power $P_{C D}=67.1 \mathrm{MW}$ to generate $I_{0}=8.03 \mathrm{MA}$ so $\gamma=0.411$. The equilibrium has a poloidal beta $\left(\beta_{p}\right)$ near unity and is very slightly paramagnetic in the center. The magnetic axis is at $4.99 \mathrm{~m}$.

For comparison, a slightly altered spectrum was chosen to duplicate the same equilibrium. In this case $P_{C D}=67.7 \mathrm{MW}$ generated $1_{0}=8.02 \mathrm{MA}$ with $B_{+}=$ 0.0362 and the same $q_{a}$ and $q_{b}$ as before. Hence, in this case $\gamma=0.406$. 
Both values of $Y$ are plotted in Fig. 8 along with the results of two other calculations at higher densities. (Al) other parameters are glven by Table 1 for steady state operation.) We note that densities of $\bar{n}_{e}=1.0 \times$ $10^{20} \mathrm{~m}^{-3}$ and $1.4 \times 10^{20} \mathrm{~m}^{-3}$ roughly correspond to half- and full-power operation of the reference INTOR. 5 it is noteworthy that $y$ increases slightly at higher density in this sequence. This is associated with a change in the current density profile at higher $\beta_{t}$, namely, the $j$ profile broadens and more rf current is generated in the relatively low denslty outer flux surface region. 7 Figure 9 illustrates the converged equilibrium at $\bar{n}_{e}=1.4 \times 10^{20} \mathrm{~m}^{-3}$ with $\beta_{t}=0.0710, l_{0}=7.98 \mathrm{MA}, q_{a}=0.99$, and $q_{b}=2.47$. This broader current profile required $P_{C D}=116.8 \mathrm{MW}$, resulting in $\gamma=0.465$. This equilibrium is diamagnetic and has $\beta_{p}=1.8$. The magnetlc axis is at $5.13 \mathrm{~m}$.

If full power operation of INTOR requires unacceptably $h$ igh $P_{C D}$ at $R_{0}=$ 4.9, consideration of a smaller major radius should be made. Figure 10 shows the linear relationshlp of $P_{C D} \sim R_{0}$ (with flxed parameters $B_{+}=0.0582, k=$ 1.6, $A=4.5, \bar{T}_{e}=18 \mathrm{keV}$ ) for a full sized reactor. (SInce the neutron wall load, $W_{n}=3.5 \mathrm{MW} / \mathrm{m}^{2}$, is higher than for INTOR the values of $I_{0}$ and $\bar{n}_{e}$ are larger at a given $R_{0}$ than for INTOR; thus $P_{C D}$ at $R_{0}=5.0 \mathrm{~m}$ is much higher than full-power operation of INTOR.) The constancy of $\gamma$ IIlustrated here suggests significant reductions in $P_{C D}$ are possible if INTOR is designed with $R_{0} \lesssim 4 \mathrm{~m}$.

The variation of $\gamma$ with $\bar{T}_{e}$ is important in light of the experlmental trend seen in Fig. 1. The electron temperature dependence in Eq. (1) does not seem simple because $\tilde{J} / \mathrm{P}_{\text {rf }}^{\sim}$ depends on the parameter $w$. However, linear Landau damping is strongest, leading to signiticant heating and current drive, only for $1 \lesssim w \lesssim 3$. In this range we find $\tilde{j} / \mathrm{p}_{\mathrm{rf}}^{\sim}$ is approximately constant, and under these circumstances we would expect $\gamma \propto \overline{\mathrm{T}}_{\mathrm{e}}$.

In order to explore the $\bar{T}_{e}$ effect on $\gamma$, we have studied five reactors for which $\bar{T}_{e}$ varied from $10 \mathrm{keV}$ to $30 \mathrm{keV}$. We held the reactor size constant $\left(R_{0}\right.$ $=5.68 \mathrm{~m}, A=6.0, k=1.6)$ and fixed the density, ion temperature, and fusion power $\left(\bar{n}_{e} \simeq 2.3 \times 10^{20} \mathrm{~m}^{-3}, \bar{T}_{i} \simeq 11 \mathrm{keV}, P_{f} \simeq 1330 \mathrm{MW}\right)$. The field strength, $B_{0}$, and current, 1 , are the only parameters besides $\bar{T}_{e}$ which are varied in this sequence, and this is done in order to keep B constant (at 0.0409) for all reactors. The results for $\gamma\left(\bar{T}_{e}\right)$, with $\bar{n}_{e}, R_{0}$, and $B$ constant, are plotted 


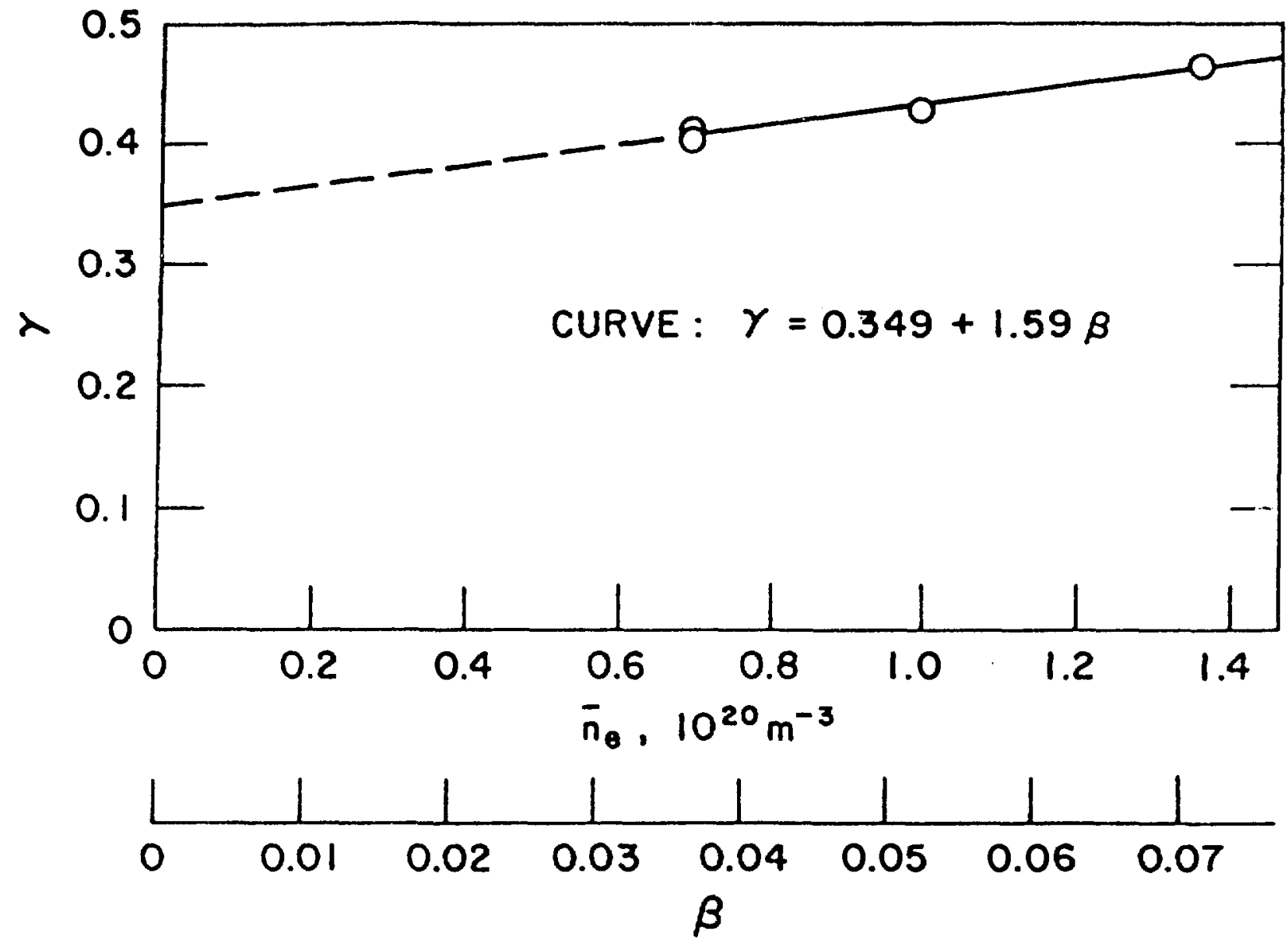

Figure 8: Current drive efficiency versus electron density with other parameters fixed; $R_{0}=4.90 \mathrm{~m}, 1=8.0 \mathrm{MA}, A=4.2, k=1.6 . a=$ $1.4, B_{0}=5.5 \mathrm{~T}, Z_{e f f}=1.5, \alpha_{n}=\alpha_{T}=0.7, \overline{\mathrm{T}}_{e}=20 \mathrm{keV}, \overline{\mathrm{T}}_{i}=10$
kev. 

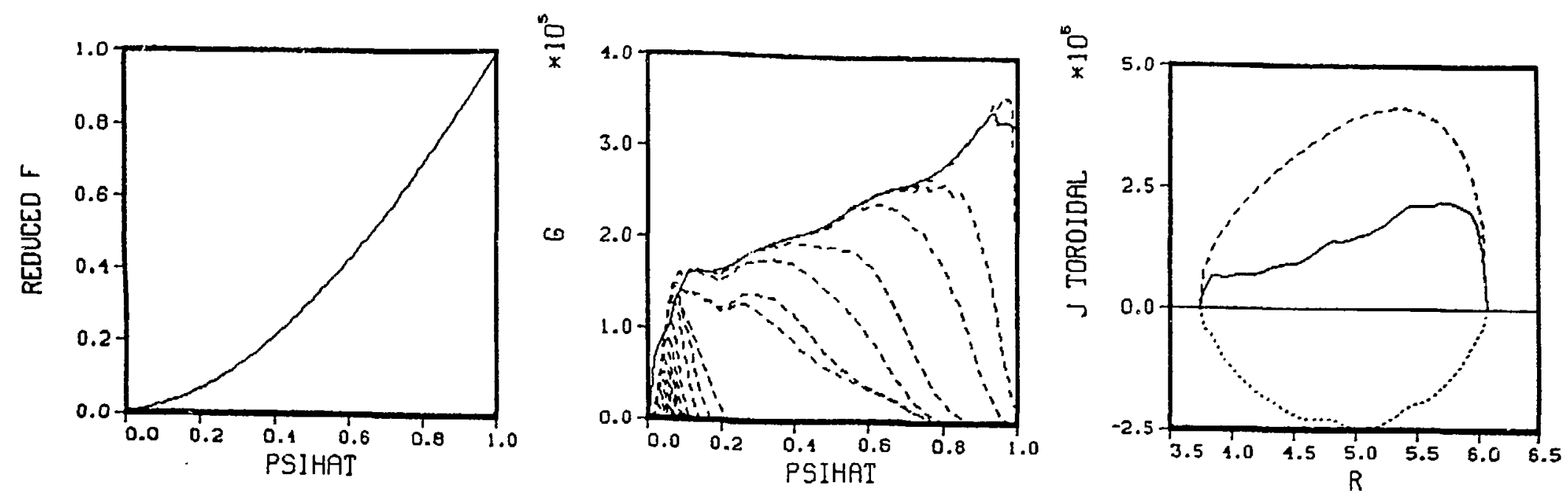

$\underline{v}$
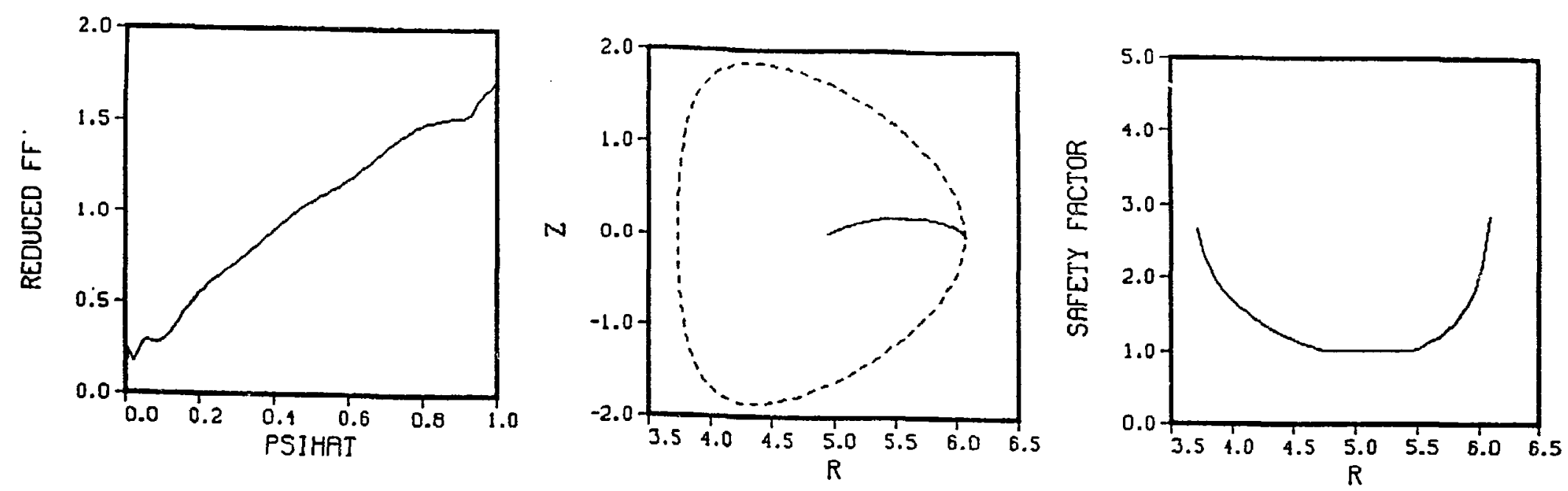

Figure 9: Equilibrium generated by HFFW for full density INTOR: $\bar{n}_{\mathrm{e}}=1.4 \times 10^{20} \mathrm{~m}^{-3}, 1 \mathrm{o}=8.0$ $\mathrm{MA}, \mathrm{T}_{e}=20 \mathrm{keV}, \mathrm{P}_{\mathrm{CD}}=117 \mathrm{MW}$, and $\gamma=0.47$. Note that the resulting $\beta_{+}=0.071$ at this high $T_{e}$ value exceeds the Troyon limit. 


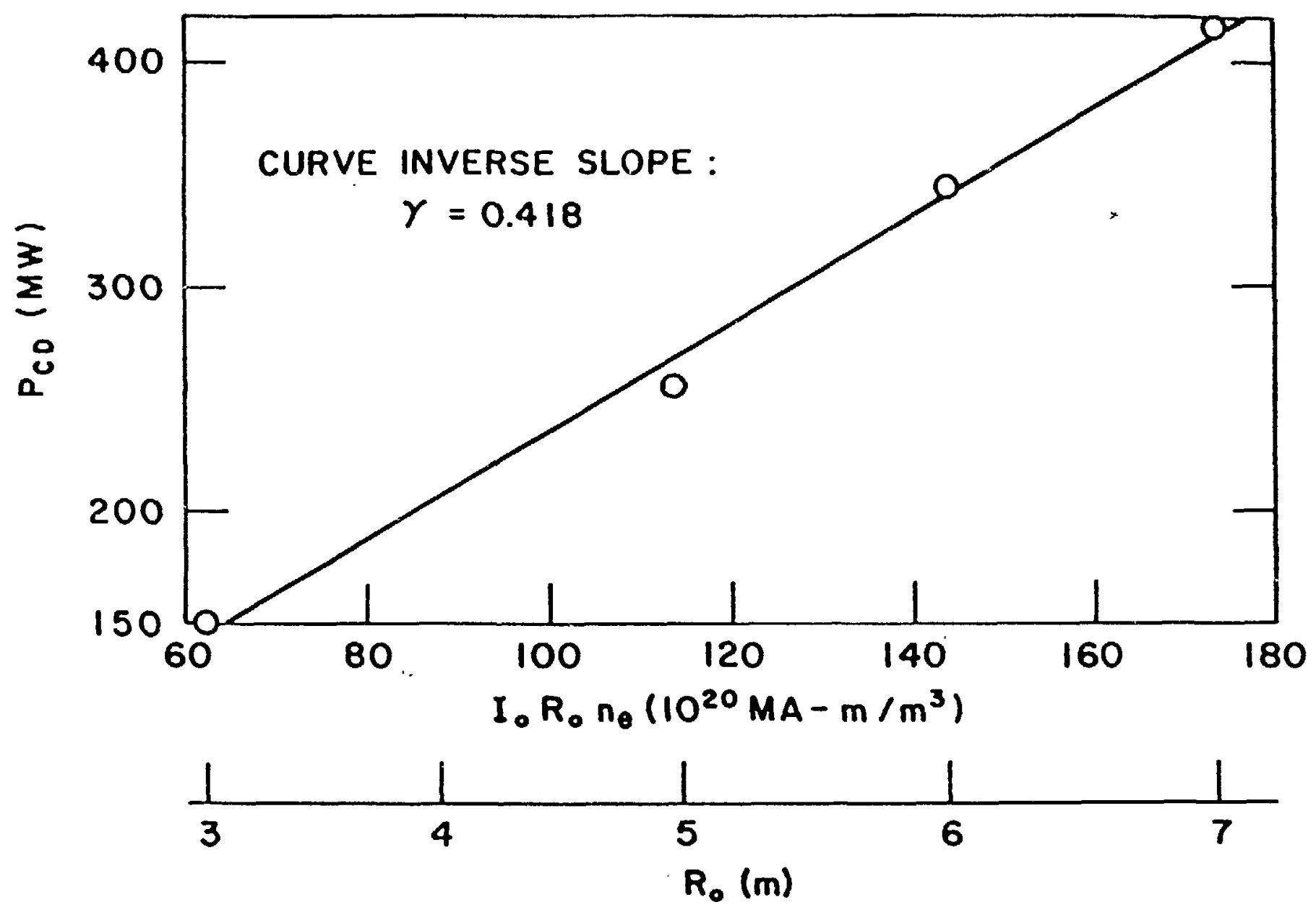

Figure 10: Current drive power versus major radius; $\beta_{+}=0.0582, A=4.5, k=$ $1.6, \alpha=1.4, Z_{\text {eff }}=1.5, \alpha_{n}=1.1, \bar{T}_{e}=18 \mathrm{kev}, \bar{T}_{i}=12 \mathrm{kev}, W_{n}=$ $3.5 \mathrm{MW} / \mathrm{m}^{2}$. 
in Fig. 11. Although $\gamma$ varies almost llnearly with $\bar{T}_{e}$ we should be careful extrapolating to values below $\overline{\mathrm{T}}_{e} \approx 10 \mathrm{keV}$. In present-day lower hybrid experiments at $\overline{\mathrm{T}}_{\mathrm{e}} \approx 1 \mathrm{keV}$ it appears that waves with $w>6$ can be strongly absorbed and drive signiflcant current in spite of the very weak damping predicted by linear theory. Reference to FIg. 6 shows these waves create $\mathcal{J} / \tilde{p}_{r f}$ much larger than the waves at $1 \leqslant w \leqslant 3$. Hence, the curve in Fig. 11 may underestimate the true value of $\gamma$, especially at low $\overline{\mathrm{T}}_{\mathbf{e}}$.

We next studied the variation of $r$ with $Z_{\text {eff }}$. Since Landau damping is strongest for $1 \leqslant w \leqslant 3$ where $\tilde{\mathrm{j}} / \tilde{p}_{r f}$ is nearly constant, we might estimate the effect of impurities on current drive by evaluating Eq. (2) at $w=2$ for different $Z$ values. Doing this for $Z=1.0,1.5$, and 2.0 , we $\mathrm{find} \mathrm{J} / \tilde{\mathrm{p}}_{\mathrm{rf}} \mathrm{In}$ the ratio 1:0.859:0.767. For comparison, we computed $Y$ for the same equilibrium $(A=6.0, k=1.6, B=0.0409,1=8.13 \mathrm{MA})$ and $p$ lasma parameters $\left(\bar{T}_{e}=18 \mathrm{keV}, \bar{n}_{e}=2.3 \times 10^{20} \mathrm{~m}^{-3}, B_{O}=8.94 \mathrm{~T}\right)$ but for different $z_{\text {eff }}$. The results, given in Table IV, have $Y$ in the ratio 1:0.856:0.772, in good agreement with the estimate.

Next we varied temperature and density profiles for a fixed pressure profile $(\alpha=1.4)$. The results, in Table $\mid V$, for the ratio of $a_{n} / \alpha_{T}$ varying from 0.27 to 3.7 show a slight increase of $\gamma$ by using peaked density and broad temperature profiles for these first stability regime equilibria. Regarding the pressure profile, we see from Table IV that the variation of $\alpha=1.4$ to $\alpha$ $=2.0$ has an insignificant effect on $\gamma$. The influence of $\bar{T}_{i}$ is expected to be small, and only a small (9\%) decrease in $\gamma$ is found (Table IV) as $\bar{T}_{i}$ increases from $12 \mathrm{keV}$ to $25 \mathrm{keV}$. At higher $\overline{\mathrm{T}}_{i}$ we expect high harmonic ion cyclotron damping to further reduce $Y$, especially for the lowest frequency waves which traverse the hot central plasma volume.

If $\gamma$ is to be useful for INTOR optimization studies it should be independent of geometry. Thus, surveys of $\gamma$ at various $A$ and $k$ values were done. All equilibria were at the limit of first regime stability. For example, the four cases with $k=1.0,1.3,1.6$, and 2.0 are displayed in Fig. 12 along with the $B_{+}$scaling of Troyor with either kink or ballooning limits. For comparison the dashed line is the Bernard scaling with geometry. The result in Fig. 13 shows again a slow increase of $\gamma$ with $B_{+}$. Note in the insets of Fig. 12 the distinctive broadening of the current profile at high 


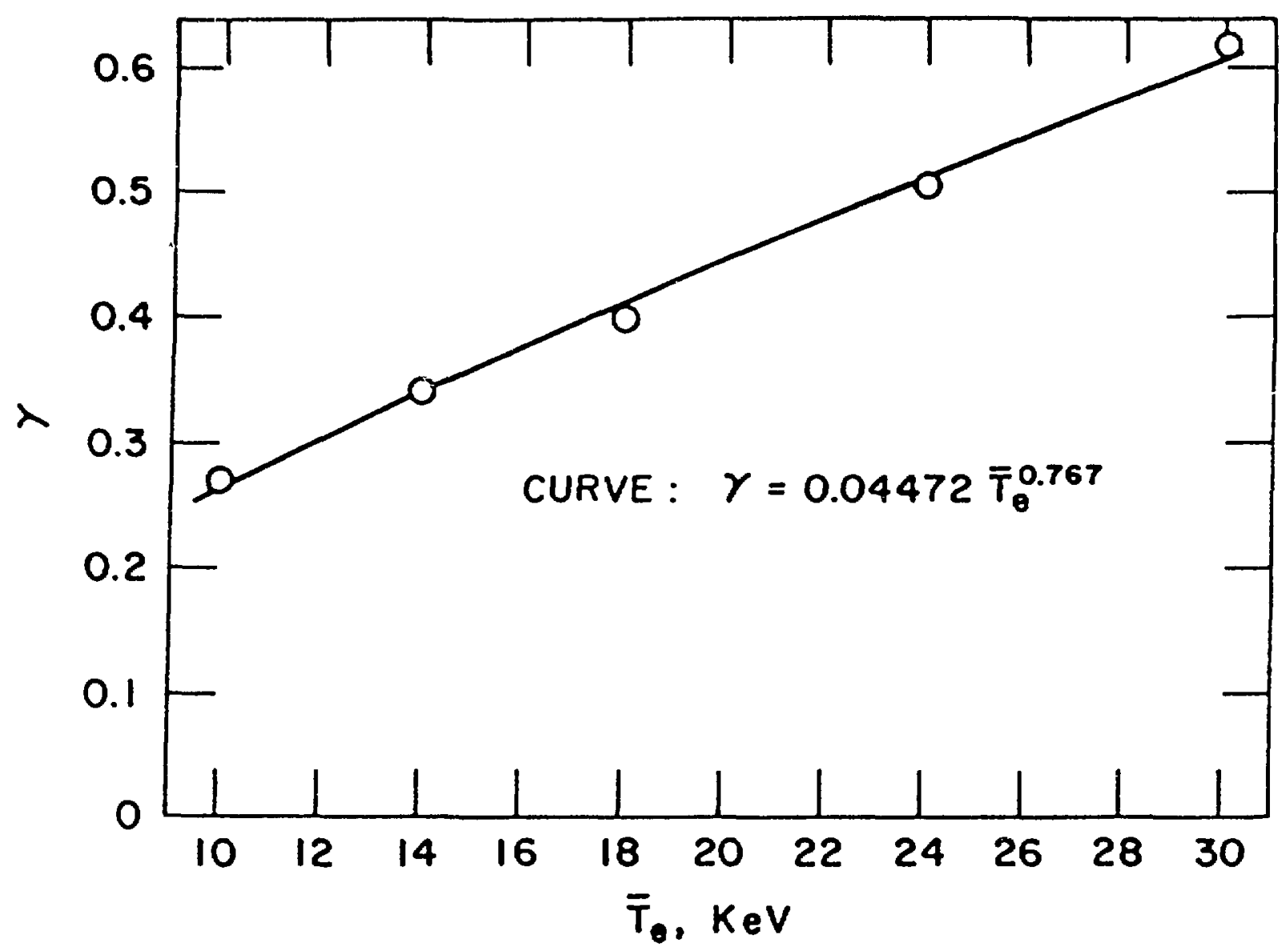

Figure 11: Current drive efficiency versus electron temperature; $R_{0}=5.68 \mathrm{~m}$, $B_{+}=0.0409, A=6.0, k=1.6, \alpha=1.4, z_{e f f}=1.5, \alpha_{n}=$ $1.1, \bar{n}_{e} \simeq 2.3 \times 10^{20} \mathrm{~m}^{-3}, T_{i}=11 \mathrm{keV}, W_{n}=3.5 \mathrm{MW} / \mathrm{m}^{2}$. 
HFFW Current Drive Sensitivity to Impurity Content, Plasma Profiles and lon Temperature

\begin{tabular}{|c|c|c|c|c|c|c|c|}
\hline$\alpha$ & $a_{n} / \alpha_{T}$ & $z_{\text {eff }}$ & $\bar{T}_{i}(\mathrm{keV})$ & $\bar{n}_{e}\left(10^{20} \mathrm{~m}^{-3}\right)$ & $1(M A)$ & $P_{C D}(M W)$ & $\gamma$ \\
\hline 1.4 & 3.7 & 1.0 & 11.7 & 2.30 & 8.17 & 229 & 0.465 \\
\hline 1.4 & 3.7 & 1.5 & 11.7 & 2.30 & 8.02 & 263. & 0.398 \\
\hline 1.4 & 3.7 & 2.0 & 11.7 & 2.30 & 8.18 & 298. & 0.359 \\
\hline 1.4 & 1.0 & 1.5 & 10.8 & 2.37 & 8.61 & 293. & 0.396 \\
\hline 1.4 & 0.27 & 1.5 & 11.1 & 2.59 & 8.58 & 365. & 0.346 \\
\hline 2.0 & 1.0 & 1.5 & 11.5 & 1.89 & 8.58 & 230 & 0.400 \\
\hline 1.4 & 3.7 & 1.5 & 17.9 & 1.64 & 7.58 & 182 & 0.389 \\
\hline 1.4 & 3.7 & 1.5 & 24.7 & 1.39 & 7.86 & 170 & 0.364 \\
\hline
\end{tabular}

Note: All cases have $A=6.0, K=1.6, \beta=0.0409, R_{0}=5.68 \mathrm{~m}$, and $\bar{T}_{e}=18.0$ kev. 


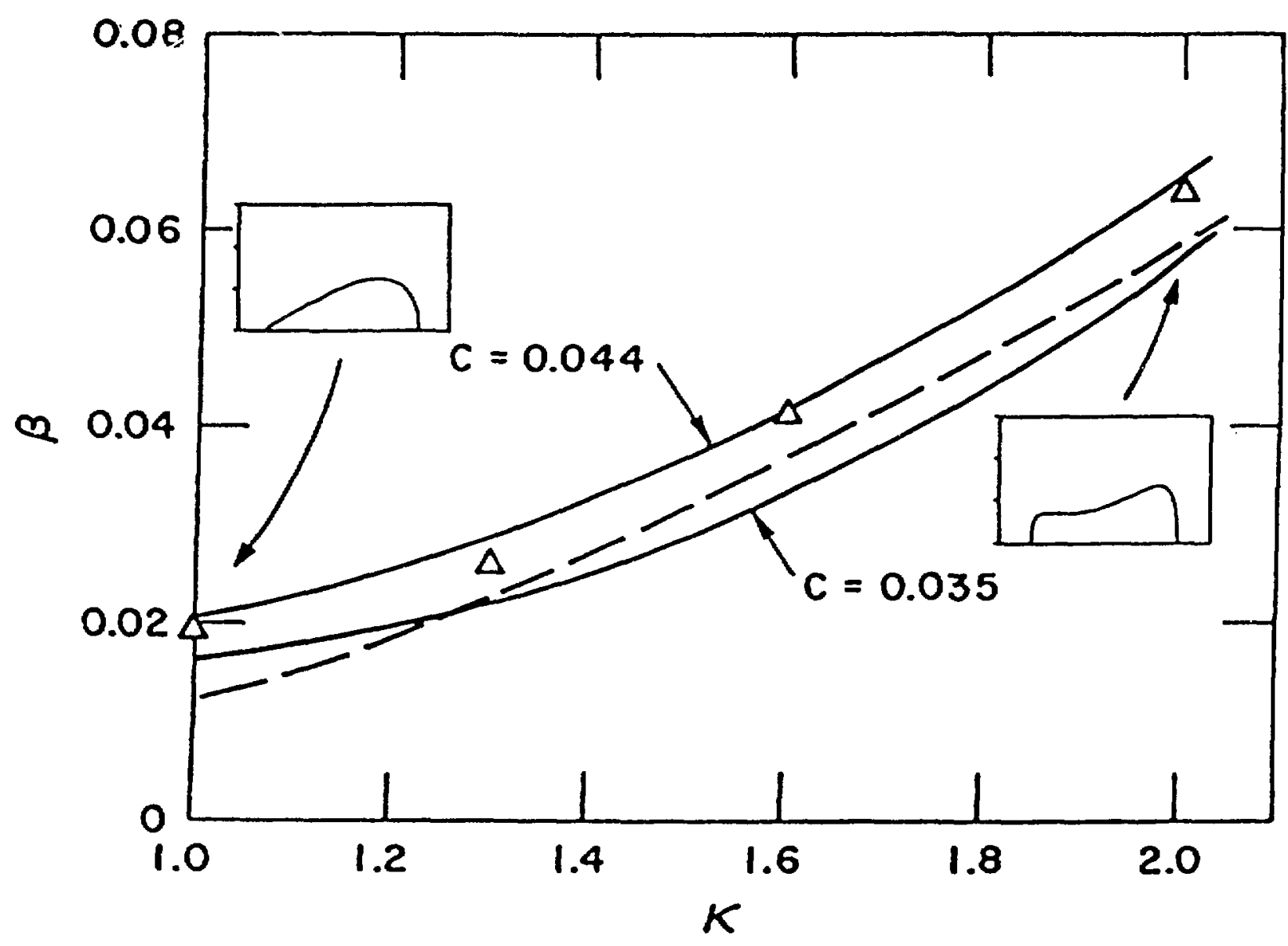

Figure 12: First stability regime limits: solid curves - Troyon formula; dashed curve - Bernard scaling. In all cases $A=6.0, \alpha=1.4,9 a$ $=1.0$, and $a_{b}=2.0$. Triangles show equilibria duplicated by HFFW current drive calculations. Insets display $j_{+}(R, 0)$. 


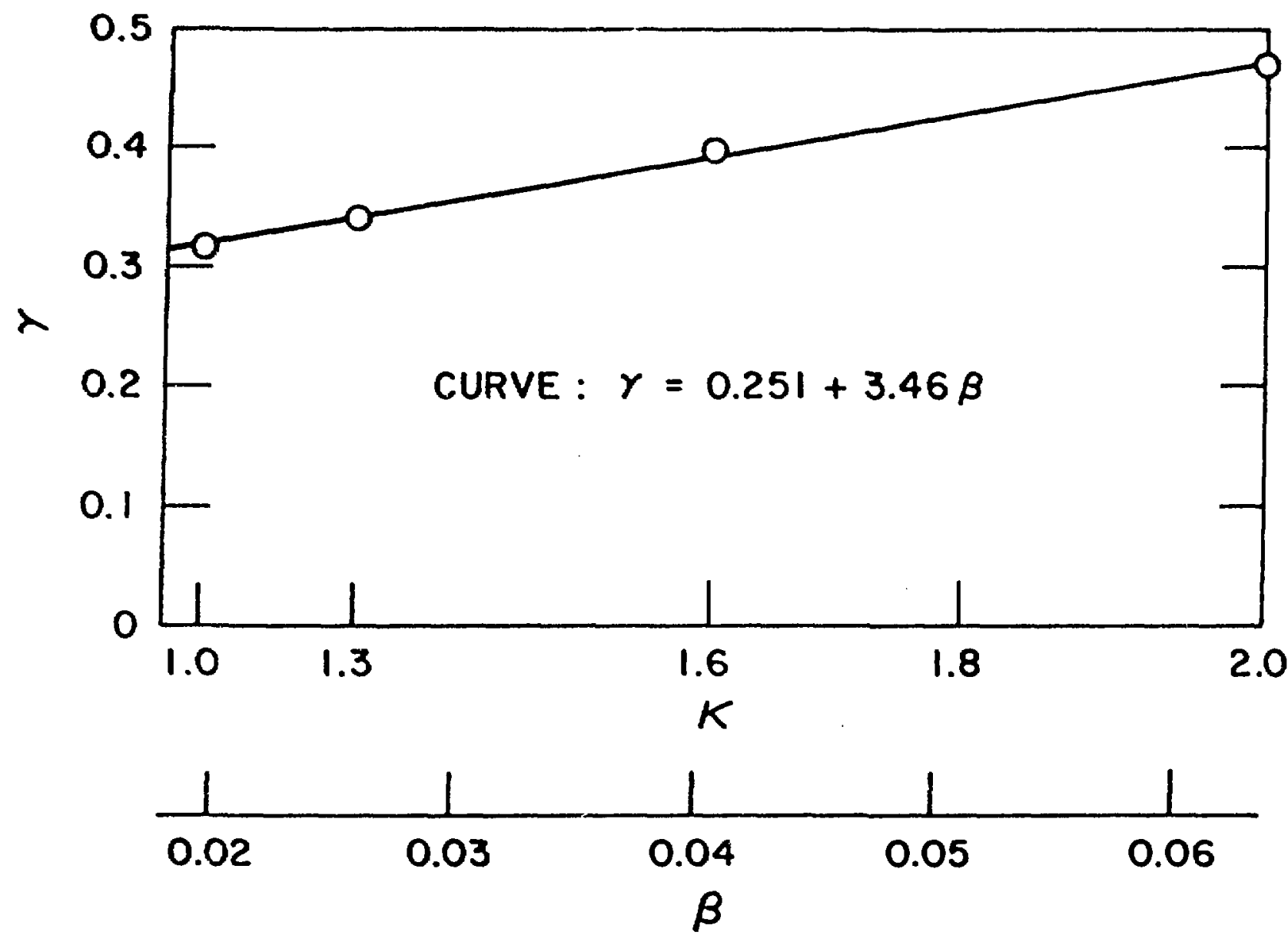

Figure 13: Current drive efficiency versus elongation; $A=6.0, \alpha=1.4, Z_{\text {eff }}$ $=1.5, a_{n}=1.1, T_{e}=18 \mathrm{keV}, \bar{T}_{i}=12 \mathrm{keV}, W_{n}=3.5 \mathrm{MW} / \mathrm{m}^{2}, P_{f}=$ $1330 \mathrm{MH}$. 
beta which is responsible for this trend. The calculations with different $A$ are plotted in Fig. 14, where a similar $\gamma\left(\beta_{+}\right)$trend is evident.

All nineteen calculations of $\gamma$ for fixed profiles, $\alpha=1.4, \alpha_{n}=1.1$, fixed $\bar{T}_{1}=12 \mathrm{keV}$, and $Z_{\text {eff }}=1.5$ can be plotted on a common graph. The result, Fig. 15, shows the increase of $\gamma$ with $\bar{T}_{e}$ and with $B$. We repeat the caution that these results are not valid for $\bar{T}_{e}<10 \mathrm{keV}$. The $\beta_{t}$ dependence of $\gamma$ may be slower than linear. The scatter of points is so large, however, that oniy a linear fit is appropriate. We should be careful not to extrapolate these results to higher $\beta_{+}$.

A curve which well fits the calculations is, for $z_{\text {eff }}=1.5$,

$$
\gamma=\left(\bar{T}_{e} / \mathrm{keV}\right)^{0.77}\left[0.034+0.196 \mathrm{~B}_{+}\right]
$$

When $Z \neq 1.5$ the value of $y$ changes in a ratio approximately proportional to

$$
\frac{\frac{32}{5+z}+2+\frac{12(6+z)}{(5+z)(3+z)}+\frac{3.76}{z}}{\frac{32}{6.5}+2+\frac{12(7.5)}{(6.5)(4.5)}+\frac{3.76}{1.5}} .
$$

For the HFFW we can summarize our conclusions.

1. For the benchmark case $\gamma=0.41$. Centrally peaked current profiles are achievable without the need for spatial diffusion of the current profile.

2. A general relationship for $\gamma$ vs. $\bar{T}_{e}, B_{+}$, and $Z_{\text {eff }}$ is given by Eqs. (3) and (4).

3. The desicn of phased waveguide arrays with fast wave polarization will require serious study in order to achieve efficient coupling.

\subsection{LFFW Current Drive}

As the wave frequency is lowered from $\sim 1 \mathrm{GHz}$ to $\lesssim 100 \mathrm{MHz}$ it may be easier to couple to the fast wave without spuriously exciting the slow wave. 


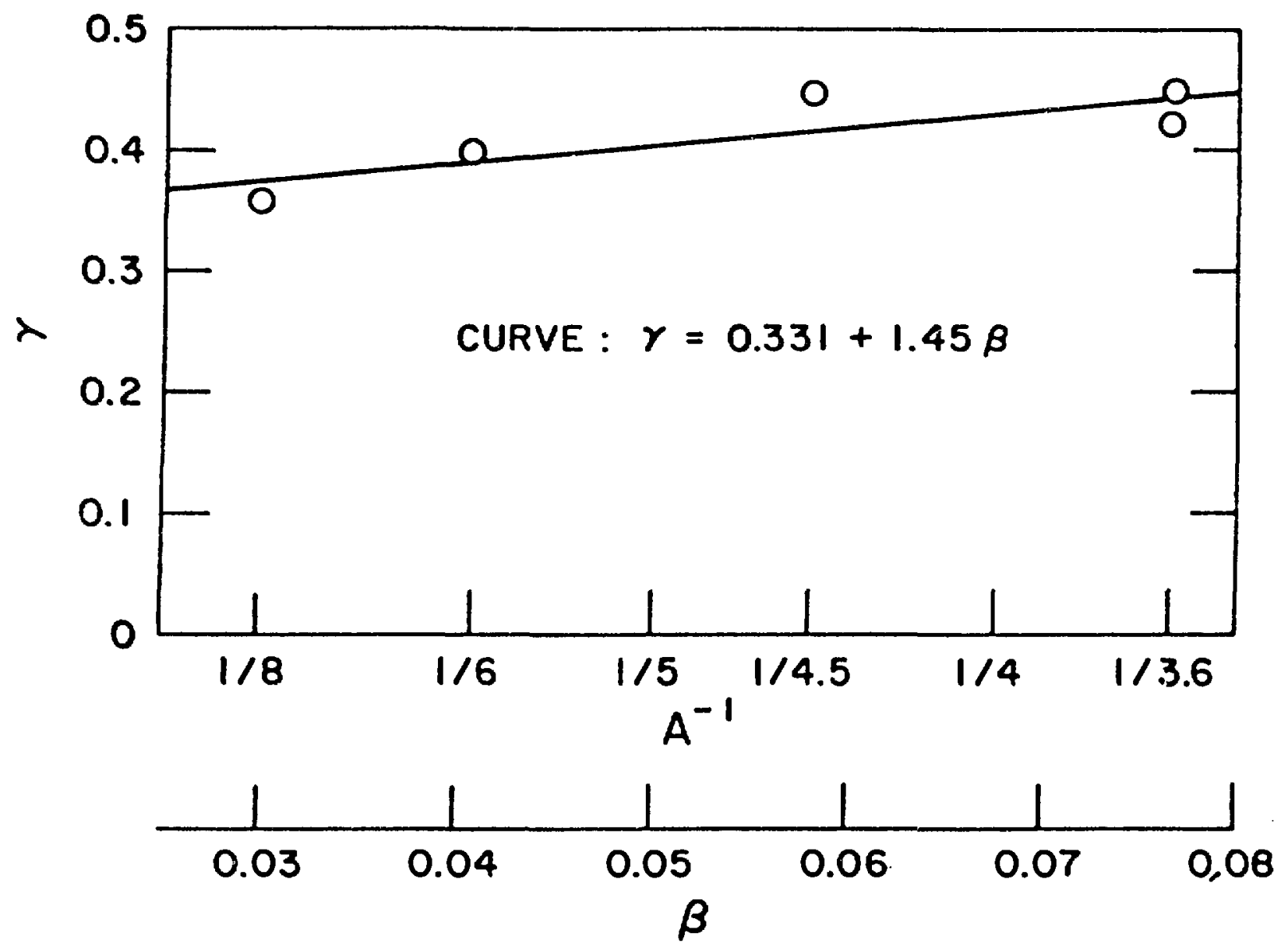

Figure 14: Current drive efficiency versus aspect ratio; $k=1.6, \alpha=1.4$, $Z_{\text {eff }}=1.5, \alpha_{n}=1.1, T_{e}=18 \mathrm{keV}, T_{i}=12 \mathrm{keV}, W_{n}=3.5 \mathrm{MW} / \mathrm{m}^{2}, \mathrm{P}_{f}$ $=1330 \mathrm{MW}$. 


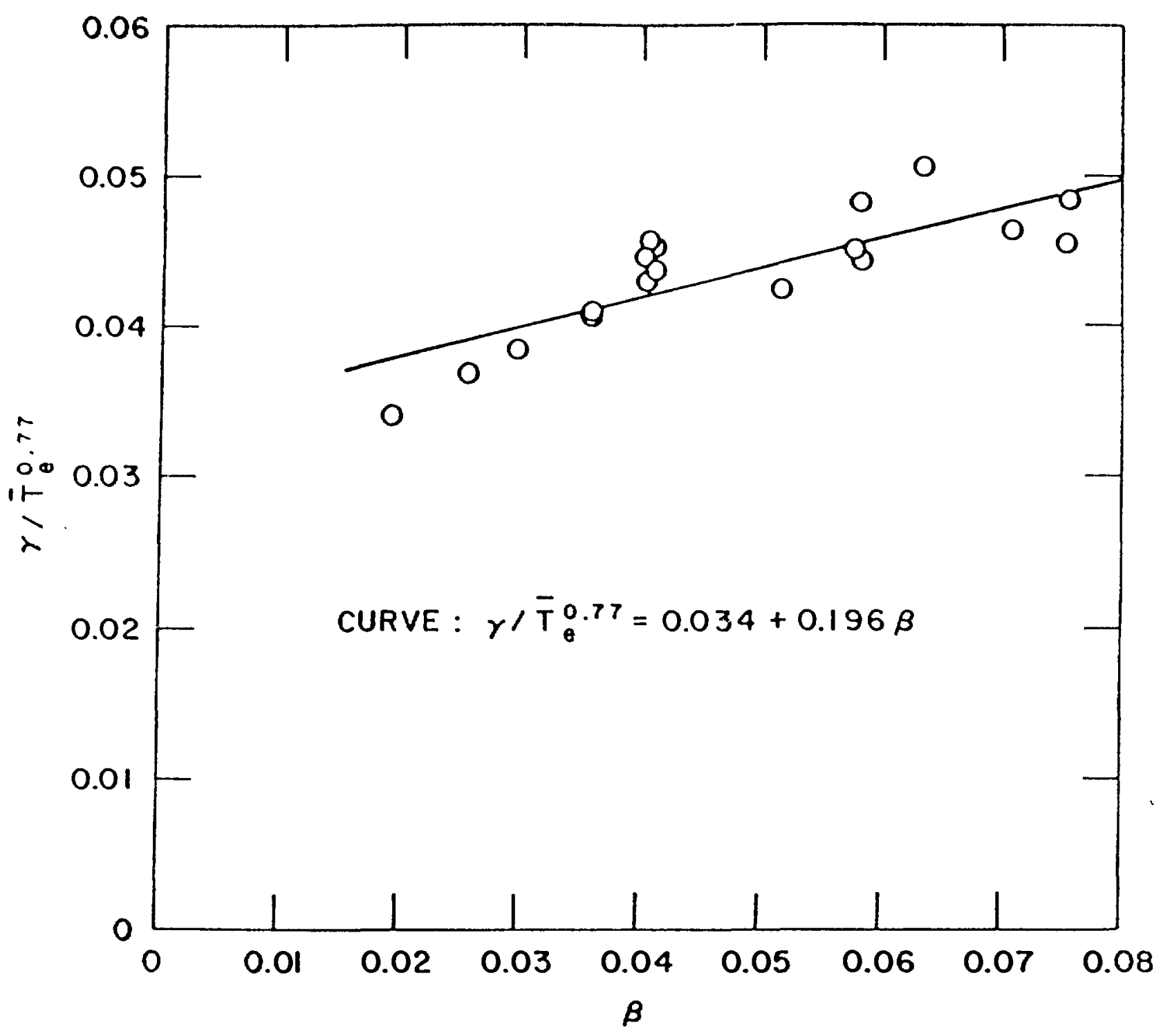

Figure 15: Universal current drive efficiency: $\alpha=1.4, Z_{\text {eff }}=1.5, \alpha_{n}=$ $1.1, T_{i} \simeq 12 \mathrm{keV}$. 
The longer wavelengths at lower frequencies tend to be insensitive to density fluctuations and edge conditlons. Ion heating (minority and second harmonic) has already been achieved, whlch demonstrates that launchers can be designed to excite the LFFW. Hopefully, in INTOR a slight tuning of the wave frequency would transfer heating from ions to electrons, so the same system might be used for auxiliary heating as well as current drive.

Minority heating is one possible technique to generate steady state current. The first two entries in Table $V$ are earlier calculations ${ }^{2,16}$ of $\gamma$ for this approach. There needs to be a disparity between $Z_{\text {eff }}$ and the minority ion charge. Thus, ${ }^{3}$ He $(Z=2)$ is appropriate if $Z_{\text {eff }} \simeq 1$, while protons $(Z=1)$ are an acceptable minority if $Z_{\text {eff }} \geq 2$. The values in the table suggest $\gamma$ increases with $\bar{T}_{e}$ and appears to be competitive with other current drive methods. However, the true value of $\gamma$ may be much lower, since trapped particle effects were ignored in Ref. 16 and 2; a study 20 of minority heating $C D$ with typical values of the local $\varepsilon \equiv A^{-1}$ showed as much as a fourfold reduction in $\gamma$ even relatively near to the magnetic axis.

An alternative LFFW method ${ }^{2}$ would employ electron Landau damping of a mode converted ion Bernstein wave. This approach avoids trapping effects. Calculations for JET (Table $V$ ) predict a higher $\gamma$ than minority heating at the same $\overline{\mathrm{T}}_{e}$.

The damping-current drive method receiving the most study for the LFFW is direct electron damping of the launched wave. As the fast wave frequency is reduced towards the ICRF it appears that. TTMP dominates over Landau damping and this increases the values of $\tilde{J} / \mathcal{F}_{r f},{ }^{19}$ as shown by Eq. 2. Previous calculations by various authors display a clear increase of $\gamma$ with $\bar{T}_{e}$, as shown by the final set of entries in Table $V$. A concern with the LFFW is avoidance of ion cyclotron damping. If $n_{1} \leqslant 2$ the electron damping is weak, so $\sim 10-50$ passes through the plasma are required to deposit wave energy in the electrons. Both thermal ions and hot alphas will compete strongly with electrons to absorb wave power. Such parasitic absorption must be avoided to maximize $r$. Thus, slower waves, with $n_{\|} \sim 5$, should be selected. The calculations in Table $V$ suggest in this case that electron damping is strong enough to effect 1-2 pass absorption. 
Table V

Previous Y Predictions for LFFW

\begin{tabular}{|c|c|c|c|c|c|c|c|c|}
\hline $\begin{array}{l}R_{0} \\
(m)\end{array}$ & $\begin{array}{c}-\overline{n_{e}} \\
\left(10^{2 O_{m}-3}\right)\end{array}$ & $\begin{array}{l}10 \\
(M A)\end{array}$ & $\begin{array}{l}P_{C D} \\
(M W)\end{array}$ & $\gamma$ & $\begin{array}{c}\bar{T}_{e} \\
(k e v)\end{array}$ & $\bar{n}_{1}$ & $\begin{array}{l}\text { Damp ing } \\
\text { Mechanism }\end{array}$ & $\begin{array}{l}\text { Ref. } \\
\therefore\end{array}$ \\
\hline 5.2 & 0.84 & 9.0 & 112 & 0.35 & 18 & -- & $3_{\text {He mi nority }}$ & 16 \\
\hline 3.0 & 0.30 & -- & -- & 0.08 & 5 & -- & $H$ minority & 2 \\
\hline 3.0 & 0.50 & - & -- & 0.15 & 5 & $\lesssim 2$ & mode conv. & 2 \\
\hline 5.2 & 0.84 & 9.0 & 82 & 0.48 & 18 & 4.6 & Landau/TTMP & 16 \\
\hline 4.2 & 1.0 & 4 & 40 & 0.42 & $\gtrsim 10$ & 2.0 & Landau/TTMP & 17 \\
\hline 5.3 & 0.7 & 6.4 & 77 & 0.31 & 10 & 5.6 & Landau/TTMP & 18 \\
\hline 3.0 & 0.6 & -- & - & 0.36 & 15 & - & Landau/TTMP & 19 \\
\hline 3.0 & 0.6 & -- & $-\infty$ & 0.09 & 5 & - & Landau/TTMP & 19 \\
\hline
\end{tabular}

1. Ref. $16^{3}$ He model: concentric circular flux surfaces $(A=4.0)$; no trapping effects; $t=40-50 \mathrm{MHz}, z_{\text {eff }}=1.2$.

2. Ref. 2: $t=33 \mathrm{MHz}$.

3. Ref. 16 model for Landau/TTMP: concentric circular flux surfaces $(A=$ 4.0); top launch, 1-2 pass absorption; $f=82 \mathrm{MHz}, Z_{\text {eff }} \simeq 1.3$.

4. Ref. 17 model: uniform infinite plasma; $10-20$ pass absorption; $f=62$ $\mathrm{MHz}$.

5. Ref. 18 model: circular flux surfaces $(A=4.1)$; top launch, 1-2 pass absorption; $f=106 \mathrm{MHz}$.

6. Ref. 19 model: $5-10$ pass absorption; $f=50 \mathrm{MHz}$. 
The actual implementation of the LFFW has been briefly addressed in two of the earlier studies. Figure 16 shows the location of fuel and impurlty cold ion harmonic resonances for an INTOR-sized tokamak.16 Superimposed on the realistic, noncircular flux surfaces is a boundary for a ray tracing calculation, with concentric circular flux surfaces centered at the magnetic axis. The antenna is located at the top between ion resonances, and the intention is to have strong electron damping in the first pass, before wave reflection and subsequent damping by the ions. Figure 17 is the result of following several rays in the assumed spectrum; over $90 \%$ of the power is absorbed by electrons before reflection off the bottom. The radial heating power density is shown in Fig. 18, where we see there is only a trivial power loss to ions. The $k$ component varies along the ray trajectories, and likewise $w=w / k_{n} v_{e}$ varies. Typically, however, $w=1-2$ throughout the bulk of the plasma, so $\mathrm{J} / \tilde{p}_{r f}$ is nearly constant, near the minimum value in Fig. 6. The current profile with this concentric circular flux surface model is shown in Fig. 19 for $\bar{T}_{e}=16 \mathrm{keV}$. The profile of $j(r)$ has the required central peaking, although additional (higher $n_{\|}$) rays might be needed if a broader $j(r)$ were desired. We find that Ref. 18 also employs a nearly identical approach with top launch between the third and fourth tritium harmonics.

For the steady state INTOR benchmark calculation (Table I) with the LFFW we used the coupled RF-MHD code ${ }^{7}$ described in Sec. 2.3, and we chose $Z_{\text {eff }}=$ 1.5. The dispersion relation was specifically modified to consider waves with $\omega \gtrsim \Omega_{i}$, where $\Omega_{i}$ is the ion cyclotron frequency. Presently the code only simulates midplane launch, so $\omega=2 \pi f$ is chosen to remove the cold fuel ion resonances from the low-field side of the magnetic axis. The ion resonances are shown in Fig. 20 for $f=64 \mathrm{MHz}$, along with the poloidal projection of a typical ray path. The radial group velocity is very large and the toroidal path length is quite short. The mode illustrated, with $n_{\|}=3.5$, passes through the magnetic axis and, as shown in Fig. 21, deposits $80 \%$ of its power into the electrons on the way $i n$. After passing the axis the ray continues to propagate to higher magnetic field until it is completely absorbed at the $\omega=2 \Omega_{T}$, tritium harmonic layer. Less than $10 \%$ of the power goes to tritium heating. As with the HFFW it is advantageous to add some LH current drive near the plasma surface, and the power spectrum for the benchmark calculation is as follows: 


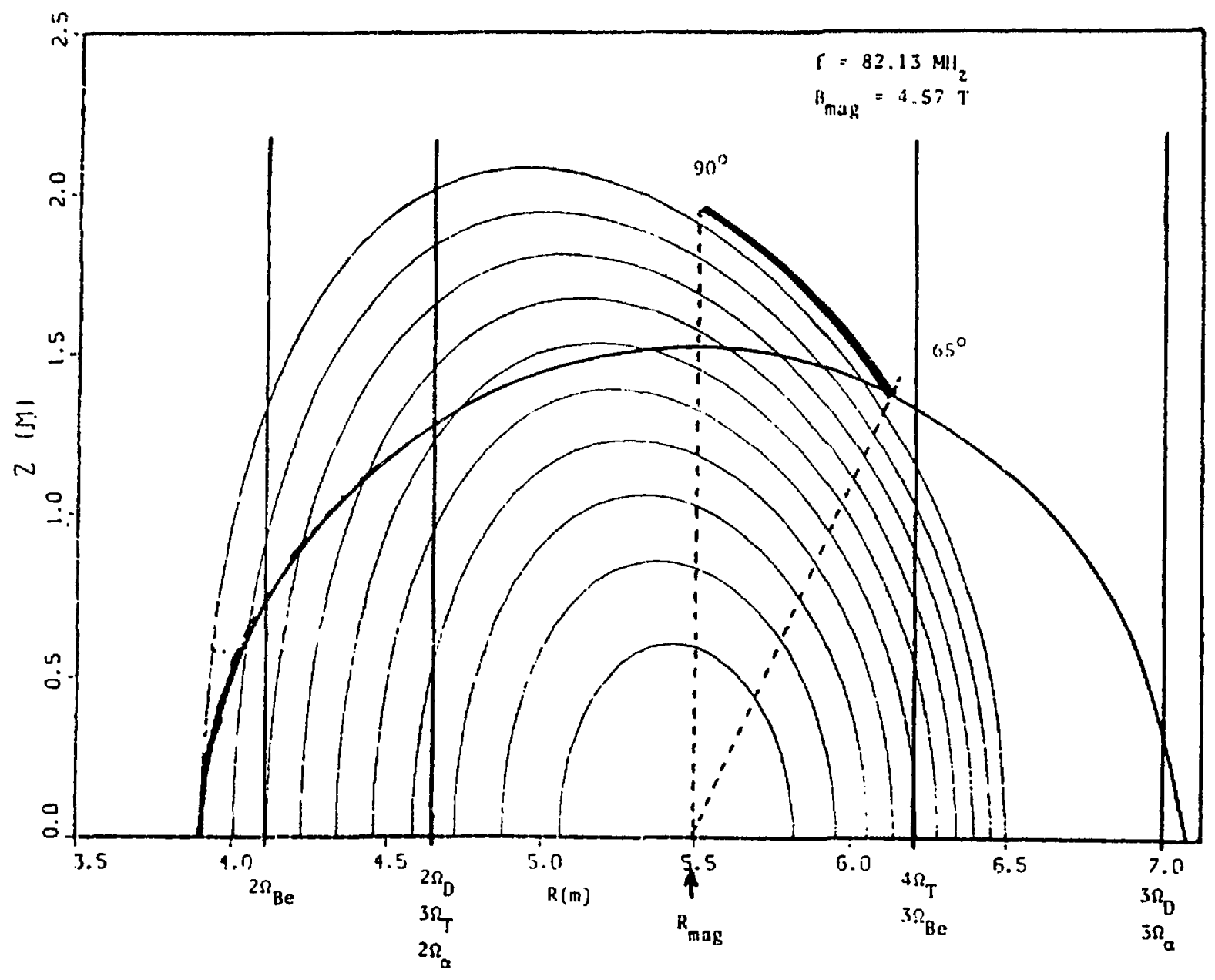

Geometry for ray tracing calculation

Figure 16: Top launch configuration typical of LFFW current drive. 


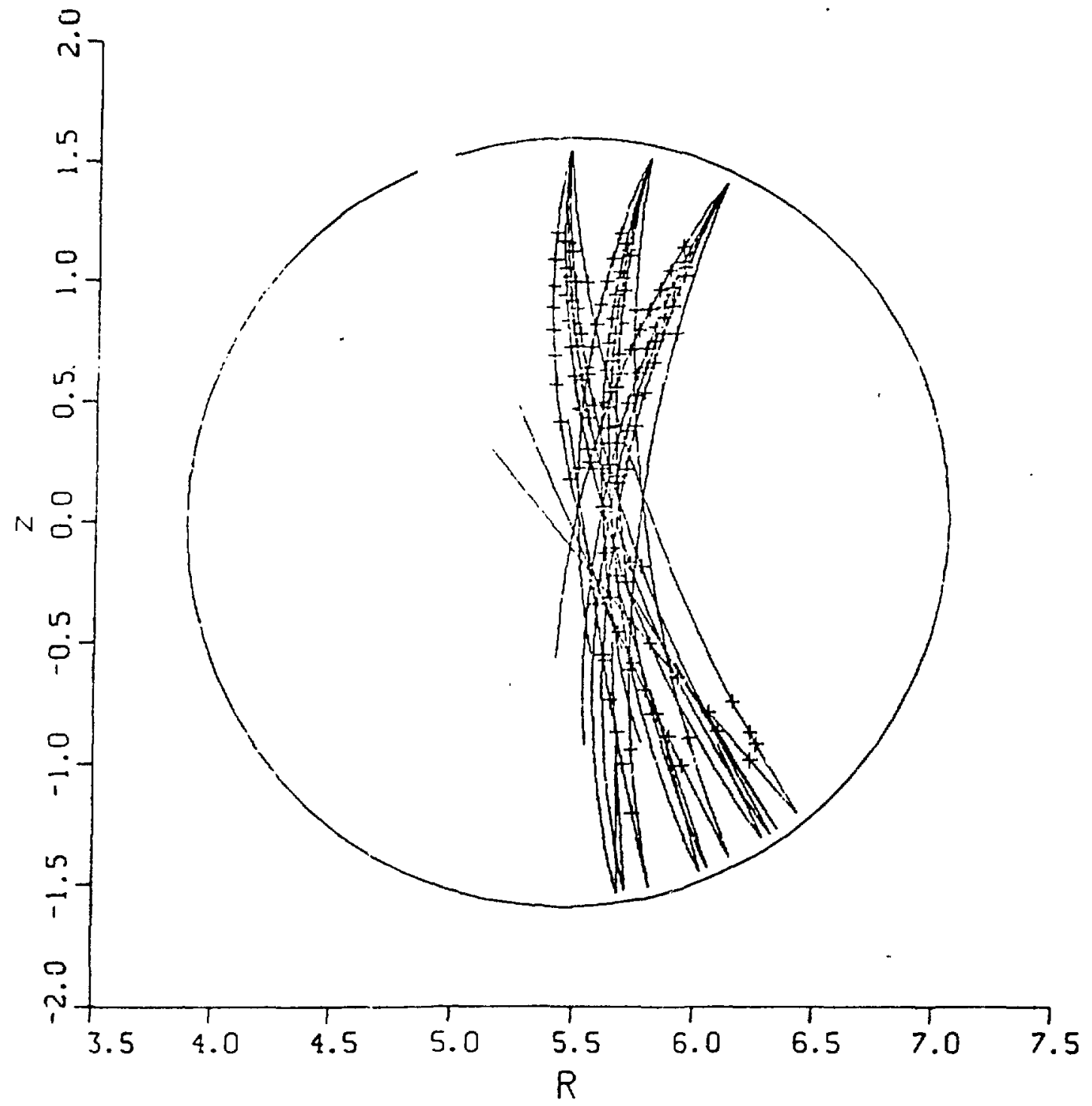

Sample ray trajectories.

Figure 17: Ray paths for a broad poloidal mode spectrum launched from the antenna in Fig. 16. Crosses denote 5\% damping decrements. 


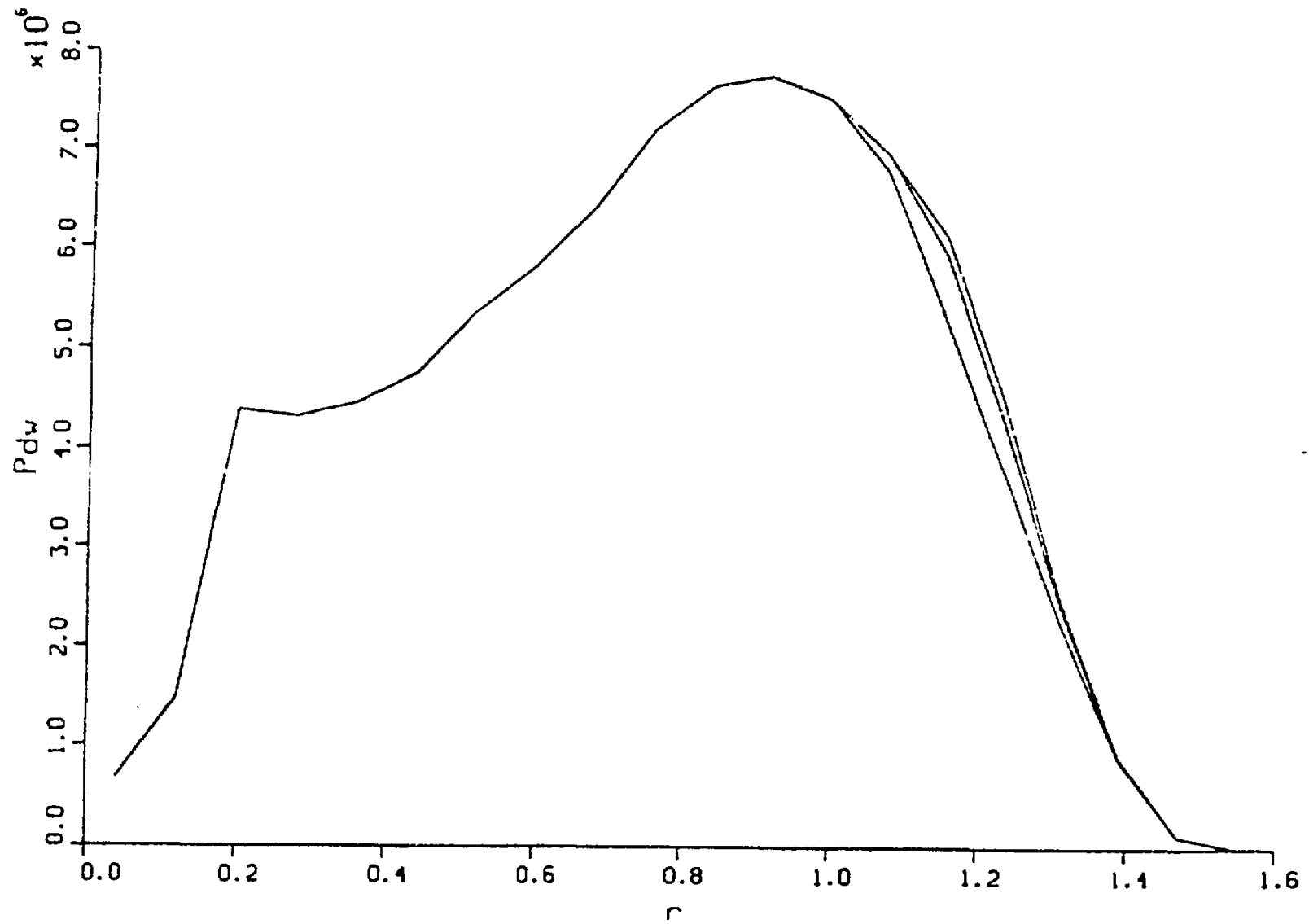

Figure 18: Wave radial power dissipation; bottom curve is power absorbed by electrons, remaining increments are to tritium and beryllium. 


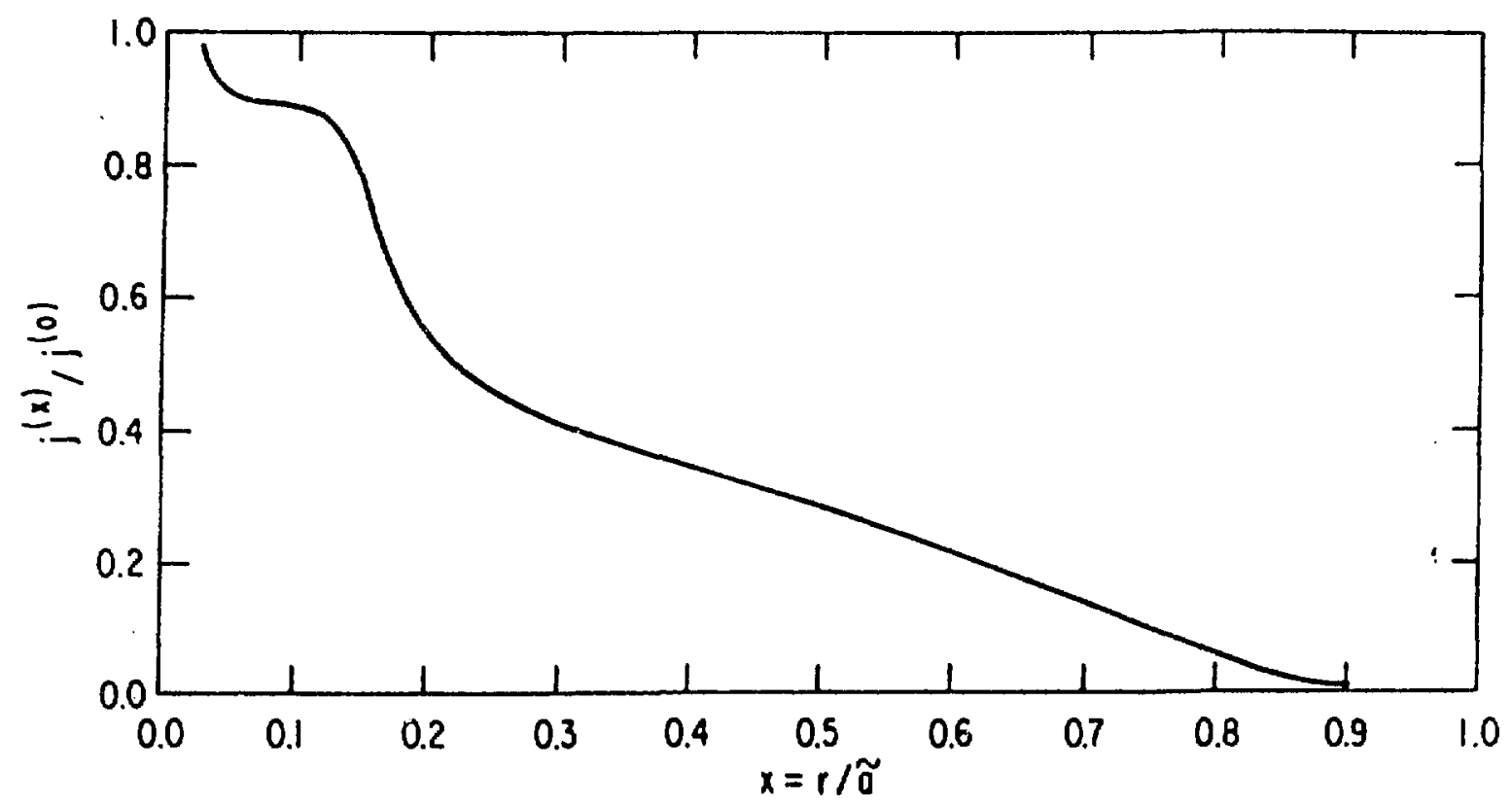

Figure 19: Current density profile for HSMS at $82 \mathrm{MHz}$ and $\lambda_{1}=0.8 \mathrm{~m}$. 

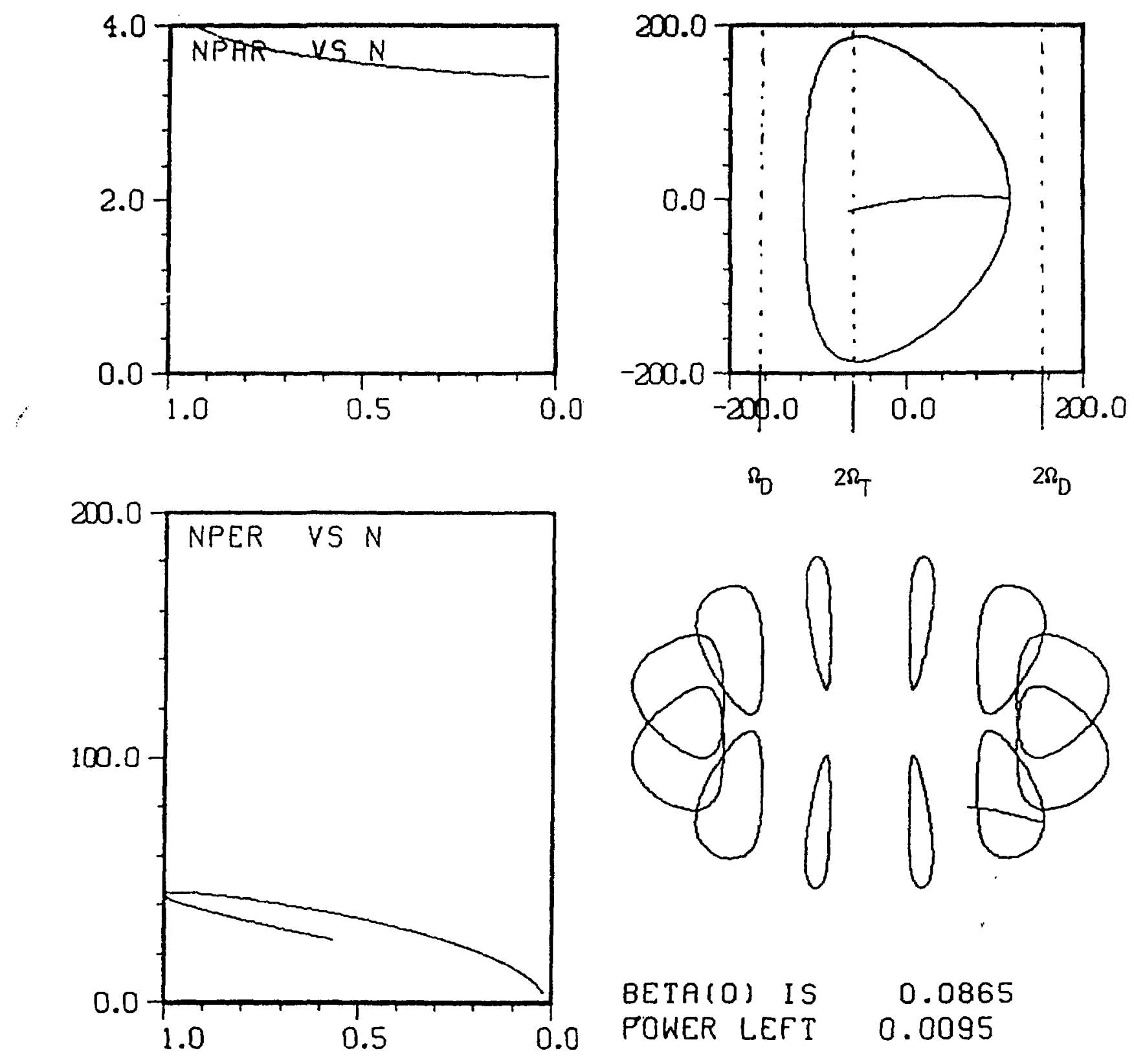

$\Omega_{0} \quad 2 \Omega_{T}$

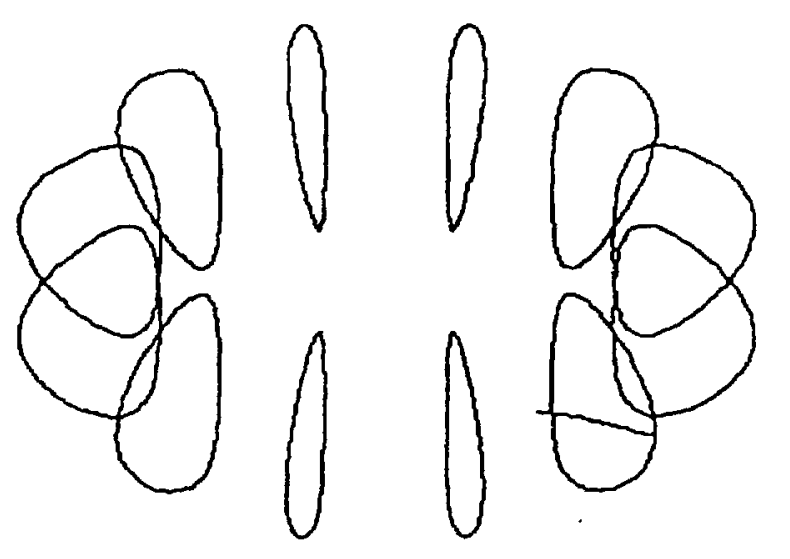

BETA(O) IS 0.0865 POWER LEFT 0.0095

Figure 20: Typical ray history for benchmark LFFW: $f=64 \mathrm{MHz}, n_{1}=3.5$, $T_{\text {eo }}$ $=35 \mathrm{keV}, n_{\mathrm{eo}}=1.23 \times 10^{20} \mathrm{~m}^{-3}$, and $T_{i o}=18 \mathrm{keV}$. Note spatial locations of ion resonances for $A=4.2, B_{0}=5.5 \mathrm{~T}$. 

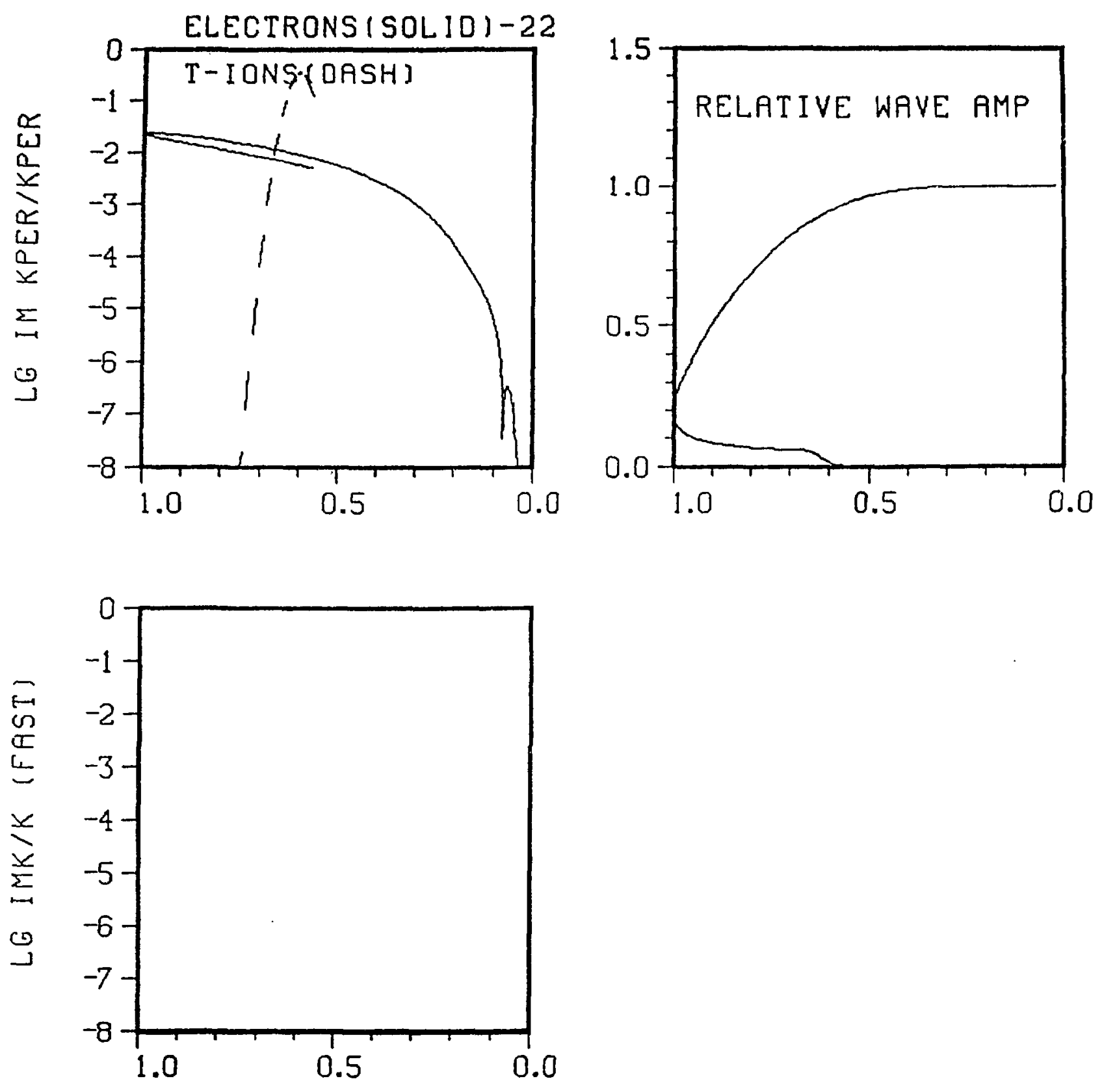

Figure 21: Same ray as in Fig. 20. Ion damping only occurs when the ray reaches the $\omega=2 \Omega_{T}$ radius, but over $90 \%$ of the wave power has al ready been dissipated on electrons by that time. 


\begin{tabular}{ccccc} 
Polarization & Frequency $(\mathrm{GHz})$ & & $n_{\mathbf{L}}$ & Edge Power $(\mathrm{MW})$ \\
\cline { 2 - 2 } LH & 2.0 & & 2.0 & 1.0 \\
LH & 2.0 & 1.8 & 2.0 \\
LH & 10.0 & 1.6 & 4.0 \\
FW & 0.064 & 3.00 & 20.0 \\
FW & 0.064 & 3.25 & 10.0 \\
FW & 0.064 & 3.50 & 5.0 \\
FW & 0.064 & 4.00 & 5.0 \\
FW & 0.064 & 4.50 & 5.0
\end{tabular}

The cumulative contributions of these rays to $G(\psi)$ is shown in Fig. 22 . Considerable current spreading is used (averaging over $50 \%$ of the points) to smooth the $G$ function (solid line in the figure). This spectrum converges to an MHD equilibrium with $\beta_{+}=0.031, a_{a}=0.74, q_{b}=3.2, \bar{n}_{e}=0.59 \times 10^{20} \mathrm{~m}^{-3}$, $\vec{T}_{e}=16.9 \mathrm{keV}$, and $\mathrm{l}_{0}=5.9 \mathrm{MA}$. The total power is $P_{C D}=52 \mathrm{MW}$, so $\gamma=$ 0.33. The current profile in Fig. 22 may not be stable; moreover $j$ should be broader to keep $a_{a} \geq 1.0$ and to allow $1_{0} \simeq 8.0 \mathrm{MA}$. Further adjustments will be necessary to refine this LFFW calculation and create an equilibrium appropriate to stable INTOR operation. Nevertheless, the calculated $\gamma$ is probably reasonably representative of what to expect for the benchmark case.

We summarize the LFFW option as follows:

1. The benchmark case should yield roughly $\gamma \simeq 0.33$, which is consistent with values found in previous studies. We expect a monotonic increase of $\gamma$ with $\overline{\mathrm{T}}_{\mathrm{e}}$.

2. Improvements in the future should include larger n, modes and differing $T_{e}(\tilde{\psi})$ profiles to assess the possibility of duplicating smooth current profiles associated with low beta equilibria.

3. In order to achieve single-pass electron damping the $n_{n}$ values must be large. Also, off-midplane launch will introduce additional capabilities of avoiding ion damping. 

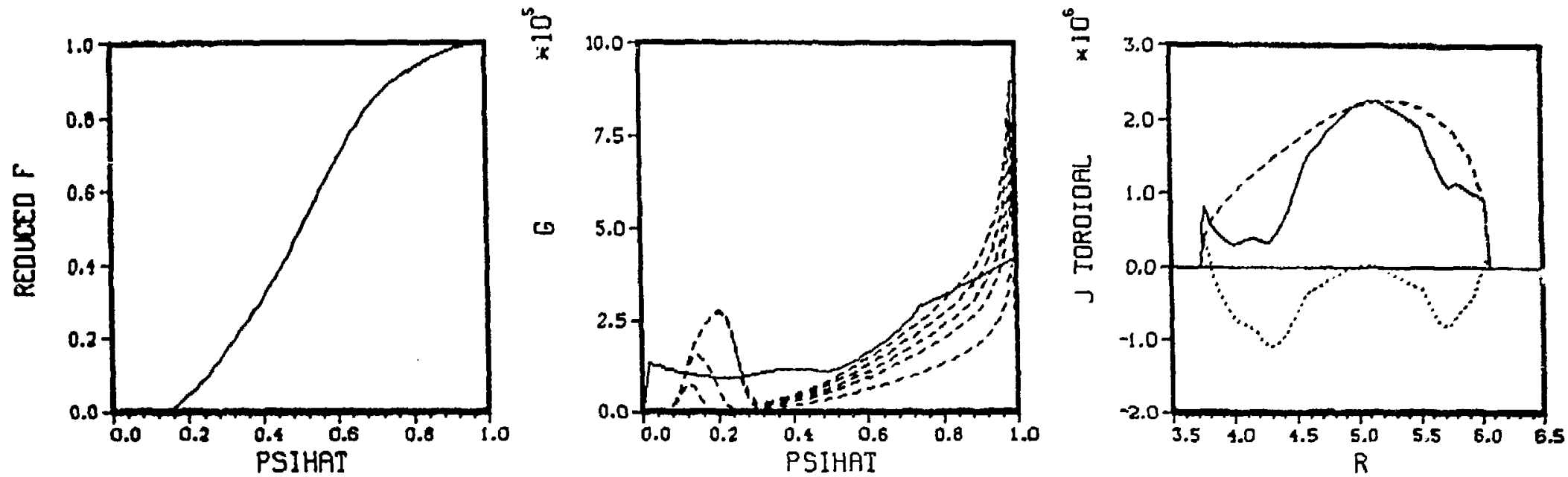

$\pm$
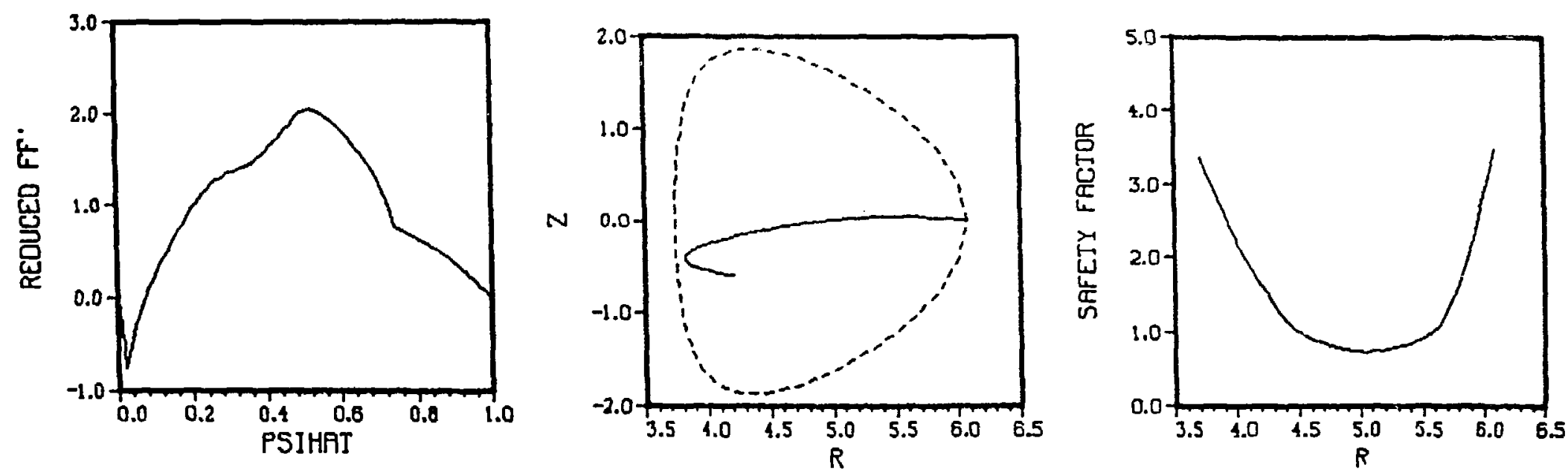

Figure 22: Benchmark INTOR with LFFW equilibrium generated with $f=64 \mathrm{MHz}, 3.0 \leq n_{1} \leq 4.5, P_{C D}$ $=52 \mathrm{MW}, \mathrm{I}_{0}=5.9 \mathrm{MA}, \bar{n}_{e}=0.59 \times 10^{20} \mathrm{~m}^{-3}, \bar{\tau}_{e}=17 \mathrm{keV}$, and $\beta_{+}=0.031$, for which $\gamma$ $=0.33$. 


\subsection{B Current Drive}

In order to understand the effects of $z_{e f f}$ and neoclassical trapping, which, unllke for wave driven currents, can be helpful for maximizing $\gamma$, we briefly review the calculation of NB current drive.

Upon Injection of a neutral beam into the plasma the atoms are ionized and circulate toroidally, their radial deposition profile being determined by the plasma density and temperature profiles and by the neutral atom velocities. The circulating ions constitute a plasma current; however, momentum transfer to electrons pushes the electrons in the same direction, creating a reduction in the net current. In toroidal systems a fraction of the electrons are trapped; these do not contribute to the counter current if they receive momentum, and they also increase the friction on the circulating electrons. Thus toroidal effects can act to increase the net current relative to a straight field geometry. These effects are included in the approximate current density expression in terms of the fast ion (beam) current density, $j_{b}, 22$

$$
j=j_{b}\left\{1-\frac{Z_{b}}{Z_{\text {eff }}}\left[1-1.46 \sqrt{\varepsilon}\left(1+\frac{0.7}{Z_{\text {eff }}}\right)\right]\right\},
$$

where the subscript $b$ refers to the fast ion charge state. We define the local flux surface aspect ratio as $\varepsilon^{-1}$ and note that this formula is valid only for $\varepsilon<1$, i.e., near the magnetic axis where the current density is highest. In addition, this result assumes fast ion velocities, $v_{b}$, much less than the electron thermal speed, $v_{e}=\sqrt{T_{e} / m_{e}}$, which is the regime of present interest.

For NB current drive the dimensionless quantity $\mathcal{J} / \tilde{\mathrm{p}}$ in Eq. (1) is a function of $v_{b} / v_{e}$, and we display a typical beam driver result in Fig. 23. The figure demonstrates that there is an optimum beam energy, $v_{b} / v_{e} \approx 0.2$, which maximizes $\tilde{\jmath} / \tilde{p}$. Furthermore, when $Z_{b}<z_{\text {eff }}$, the neoclassical effects act to increase $\tilde{J} / \tilde{p}$. (When $z_{\text {ef } f}<z_{b}$ the opposite is true, as evidenced by Eq. (5).) The functional form of Eq. (1) demonstrates that high temperatures and low densities raise the current drive efficiency, $j / p$, for a given $\tilde{J} / \tilde{p}$. 


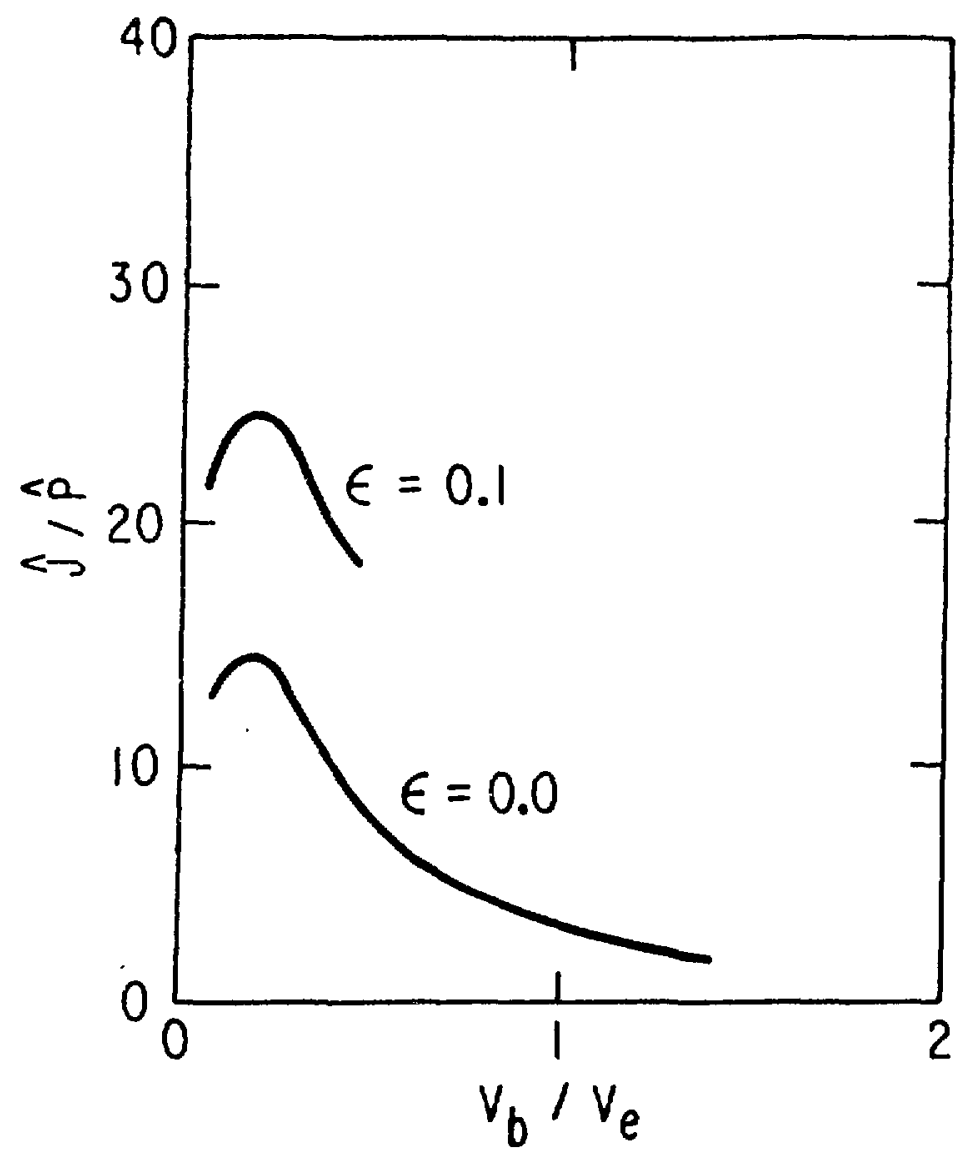

Figure 23: $D^{\circ}$ injection into $D-T$ plasma with $Z_{\text {eff }}=2$. 
Equation (1) is local in minor radius, and in Ref. 16 the calculation was generalized with a spatial Monte Carlo simulation of the beam slowing down, integrating a more accurate form of $\mathrm{Eq}$. (5) along test particle orbits:

$$
\begin{aligned}
j= & \frac{I_{B}}{N \pi K a^{2}} \sum_{b=1}^{N} Z_{b} \int_{0}^{T} s \frac{v_{\|}(t)}{2 \pi R_{0}}\left\{1-\frac{Z_{b}}{Z_{e f f}}\left[1-1.55\left(1+\frac{0.55}{Z_{e f f}}\right) \sqrt{\varepsilon}\right.\right. \\
& \left.\left.-\left(0.20+\frac{1.55}{Z_{\text {eff }}}\right) \varepsilon\right]\right\} d t,
\end{aligned}
$$

where $v_{\|}$is the fast ion toroidal velocity, 's is the slowing-down time, and $I_{B}$ is the beam current injected. The code follows the neutral atoms, computes their position of lonization, includes finite Larmor radius effects, calculates the subsequent ionization and guiding center drifts, and slows the particles down. The actual flux surfaces are modelled by concentric ellipses. Plasma temperature and density is constant on flux surfaces and in Ref. 16 the profiles are modelled as

$$
\begin{aligned}
& T(r)=T_{0}\left[1-(r / a)^{2}\right]^{1.1} \\
& n(r)=n_{0}\left[1-(r / a)^{2}\right]^{0.3}
\end{aligned}
$$

across the equatorial plane. The beam is assumed cylindrically symmetric with a Gaussian current density of half-width $20 \mathrm{~cm}$. The toroidal current density profile, $j(r)$, is sensitive to the radius of tangency, $R_{\text {tang, to the }}$ plasma; the beam axis lies close to the equatorial plane. The code integrates $j$ over the plasma cross section and computes the total current 1 for a given injection power $P_{b}$.

The most attractive regime appears to be for $z_{b}<z_{\text {eff }}$. From Eq. (5) it is obvious that $j / j_{b}$ is maximized by minimizing $z_{b} / z_{e f f}$. The authors of Ref. 16 selected a deuteron beam and increased the plasma impurity content until

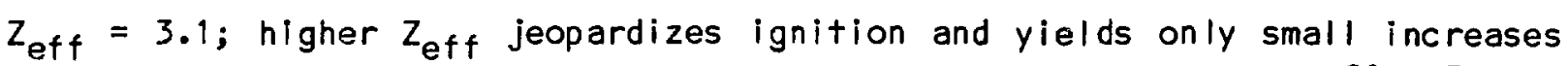
in $1 / P_{b}$. The test equilibrium had $T_{e}=18 \mathrm{keV}$ and $\bar{n}_{e}=0.84 \times 10^{20} \mathrm{~m}^{-3}$, and the optimum injection energy, $3 \mathrm{MeV}$, resulted in $\gamma=0.61$ with $R_{\text {tang }}=4.5$ 
m. (Suppression of neoclassical effects by setting $\varepsilon=0$ resulted in a lower value, $\gamma=0.48$.) See Table VI. Figure 24 displays the resulting current density profile. The current profile is very peaked around the magnetic axis.

In Ref. 21 it is noted that more conventional accelerator technology could be used it the beam energy, $E_{b}$, is kept below $1 \mathrm{MeV}$. In addition, the current profile may be broadened (compared to Fig. 24) by selecting $R_{0}<$ Rtang, withour severe shinethrough. The results for $\gamma$ at various $E_{b}$ are listed in Table VI. As expected, $\gamma$ increases with $\bar{T}_{e}$ and $E_{b}$ over the range studied.

The calculations reported here for the INTOR study were made with a Monte Carlo neutral beam deposition and fast-ion-orbit code. The neutral particles are tracked through a plasma with concentric ellipsoidal flux surfaces to determine the neutral beam deposition. The background plasma ionizes the neutrals by electron and proton ionization, charge exchange with protons, as well as ionization and charge exchange with impurity ions (ionization by fast ions is not included). The poloidal motion of the fast ions is followed as collisions with the background plasma cause energy loss and "diffusion" in pitch angle and energy. The electron current is calculated using the electron response of Start and Cordey for a finite aspect-ratio torus.

The plasma density, temperature, and current profiles are fixed for this study:

$$
\begin{aligned}
n_{e}(r) & =n_{e o}\left(1-(r / a)^{2}\right), \\
T_{e}(r)=T_{i}(r) & =T_{e o}\left(1-(r / a)^{2}\right), \\
j(r) & =j(0)\left(1-(r / a)^{2}\right) .
\end{aligned}
$$

The plasma is composed of a 50:50 $\mathrm{mix}$ of $\mathrm{D}$ and $T$, with Argon added to raise the $Z_{\text {eff }}$. An ellipsoidal toroidal vacuum vessel stops any neutral particle path which intersects it; thus, only rays with $R_{\text {tang }}>3.65 \mathrm{~m}$ make a "double pass" through the plasma. 
Table VI

\section{Previous Y Predictions for MB}

\begin{tabular}{|c|c|c|c|c|c|c|c|c|}
\hline $\begin{array}{l}R_{0} \\
(m)\end{array}$ & $\left(10^{20^{n_{e}}} \mathrm{~m}^{-3}\right)$ & $\begin{array}{l}10 \\
(M A)\end{array}$ & $\begin{array}{l}P_{C D} \\
(M H)\end{array}$ & $\gamma$ & $\begin{array}{l}\bar{T}_{e} \\
(k e v)\end{array}$ & $\begin{array}{l}E_{b} \\
(\mathrm{MeV})\end{array}$ & $\begin{array}{r}R_{\text {tang }} \\
\text { (m) }\end{array}$ & Ref. \\
\hline 5.2 & 0.84 & 9.0 & 64 & 0.61 & 18 & 3.0 & 4.5 & 16 \\
\hline 8.0 & 0.95 & 9.5 & 222 & 0.33 & 20 & 0.5 & 8.75 & 21 \\
\hline 8.0 & 0.95 & 9.5 & 154 & 0.47 & 20 & 1.0 & 8.75 & 21 \\
\hline 8.0 & 0.95 & 9.5 & 111 & 0.65 & 20 & 3.0 & 7.5 & 21 \\
\hline 8.0 & 0.59 & 9.5 & 69 & 0.65 & 33 & 1.0 & $8.0-8.5$ & 21 \\
\hline
\end{tabular}

1. Ref. 16 model: concentric elliptical flux surfaces $(A=4.0)$; neoclassical trapping included (no trapping results in $Y=0.48$ ); $D_{0}, Z_{\text {ef } f}=3.1$

2. Ref. 21 model: $A=4.0, k=1.6$; neoclassical trapping included; $D_{0}$, $Z_{\text {eff }}$ $=2.2$. 


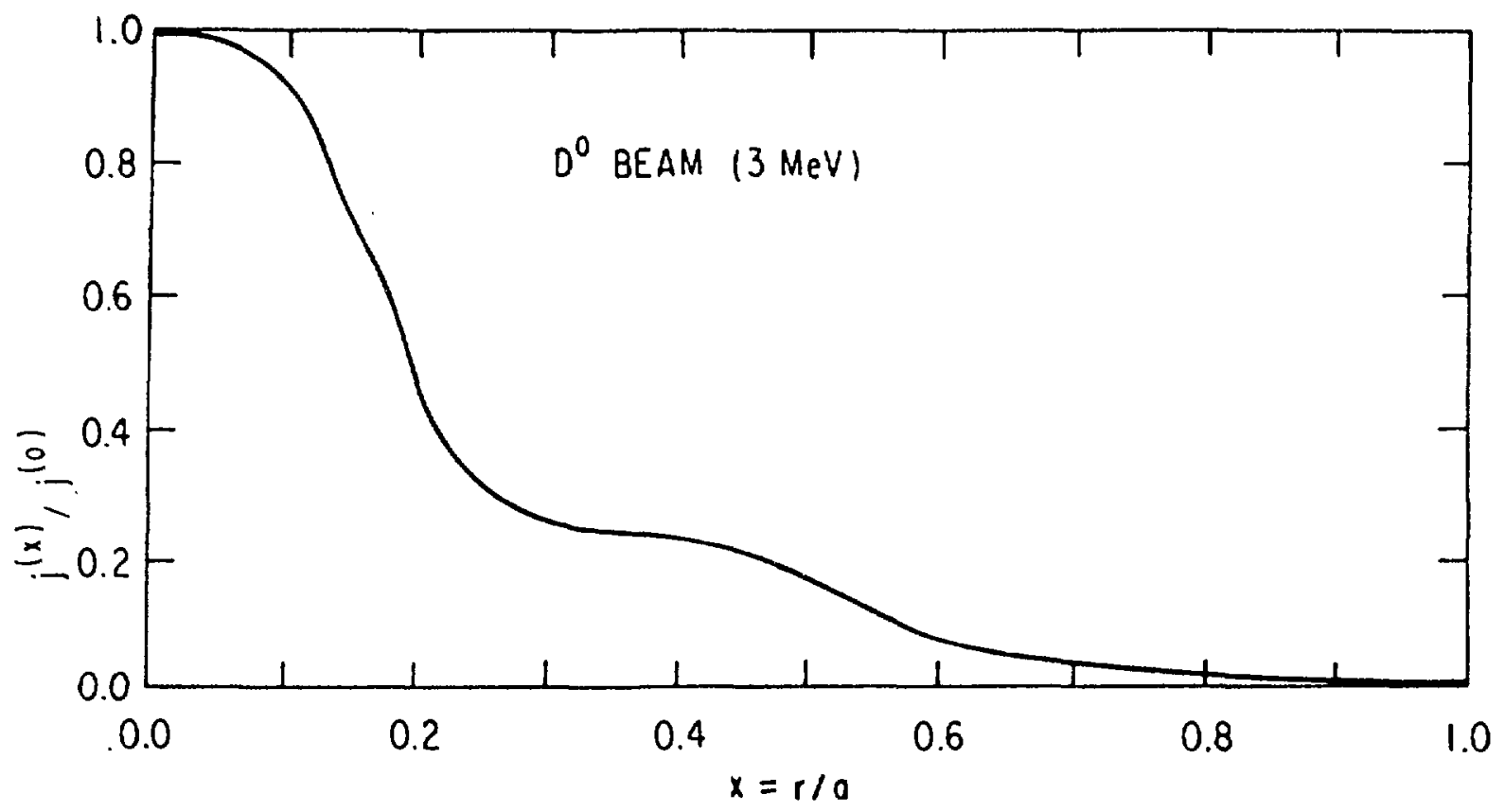

Current density profile for $3 \mathrm{MeV} \mathrm{D}^{0}$ injected into $\bar{n}_{e}=0.84 \times 10^{20} \mathrm{~m}^{-3}$ with $\mathrm{Z}_{\text {eff }}=3.1$.

Figure 24: High energy beam driven current for DEMO reactor. 
The neutral beam is modeled as a set of parallel pencil beams. The beam intensity in lts cross section is obtained from a convolution of a Gaussian function, representing the effects of divergence, and the ion source geometry, which is taken here to be of zero width and height $h_{b}$ :

$$
I(x ; y)=\int_{-h_{b} / 2}^{h_{b} / 2} \exp \left(\frac{-x^{2}-(y-h)^{2}}{2 \sigma^{2}}\right) d h
$$

where $\sigma=10 \mathrm{~cm}$. The centerline of the beam lies in the tokamak equatorial plane and its tangency radius, $R_{\text {tang, }}$ is the minimum major radius along the path of the centerline. All of the neutrals are injected with the full beam particle energy, $E_{b}$.

For the benchmark calculation we selected $Z_{\text {eff }}=2$ and $D^{\circ}$ injection with $E_{b}=0.75 \mathrm{MeV}$ and $R_{\text {tang }}=4.2 \mathrm{~m}$. Setting $\bar{n}_{e} \simeq n_{e o} / 2$ and $\bar{T}_{e} \simeq T_{e o} / 2$, we find $\gamma$ $=0.37$ for the benchmark case.

Besides the benchmark calculation, a survey if $\gamma$ sensitivity to $\bar{n}_{e}, \bar{T}_{e}$, $Z_{\text {eff }}, E_{b}$, $R_{\text {tang, and }} h_{b}$ was undertaken. For these studies the parameters were varied from a reference point which was the same as the benchmark, except $\overline{\mathrm{T}}_{\mathrm{e}}=$ $15 \mathrm{keV}$; see Table VIl. The benchmark (in boldface) and reference case (underlined) are included in Table VIII along with results of the survey.

The density dependence is summarized as $\gamma=$ constant, as $\bar{n}_{e}$ varies by a factor of three.

The temperature dependence is nearly linear, $\gamma \sim T_{\text {eo }}$, for $T_{\text {eo }}<30 \mathrm{keV}$, with saturation at higher temperatures. The optimum $Z_{\text {eff }}$ is in the range of 1.5-2.5. The dependence on beam voltage is slower than linear, $Y \sim E_{0}^{0.5}$. As $R_{\text {tang }}$ is reduced below $R_{0}$ - $a / 2$ the current drive efficiency drops rapidly because much of the beam is deposited in cold plasma; when the beam begins to strike the inner wall of the vacuum vessel $Y$ drops even more rapidly. In order to optimize the current drive efficiency it is important that $R_{\text {tang }}$ be greater than $R_{0}-a / 2$ so that the fast ions start with as much $v_{\|}$as possible. As $h_{b}$ is raised to $2.4 \mathrm{~m}$ the driven current profile broadens, but there is little reduction in $\gamma$. 
Table VII

Tok anak, Plasma, and Beam Description

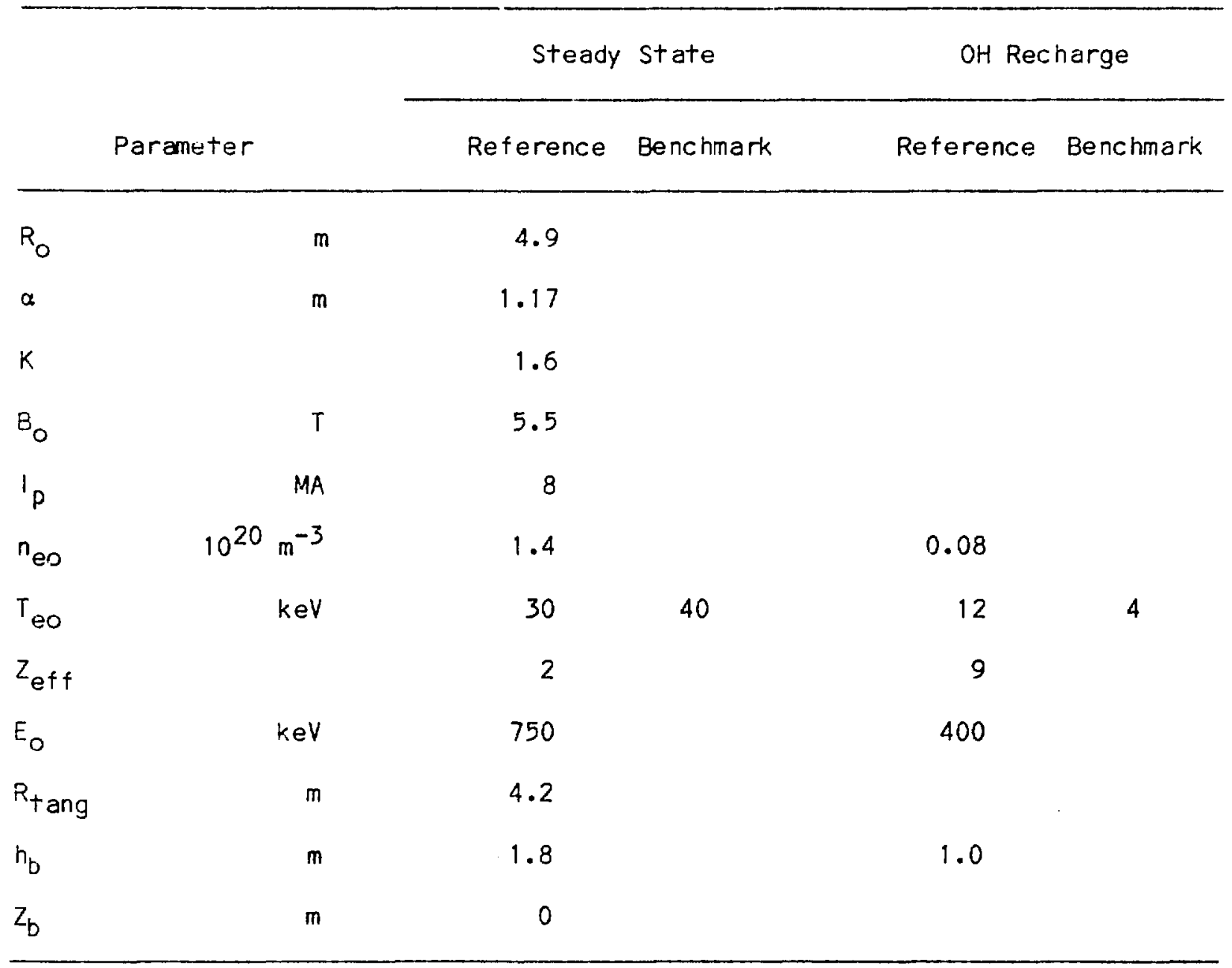




\section{NB-Driven Steady State}

\begin{tabular}{|c|c|c|c|c|}
\hline \multicolumn{2}{|c|}{ Scan } & \multirow{2}{*}{$\frac{\begin{array}{c}\eta_{C d} \\
(A / W)\end{array}}{0.217}$} & \multirow{2}{*}{$\frac{\left(10^{20} \mathrm{~A} / \mathrm{W} \mathrm{m} \mathrm{m}^{2}\right)}{.32}$} & \multirow{2}{*}{$\begin{array}{r}\begin{array}{c}P_{b} \\
(M W)\end{array} \\
37\end{array}$} \\
\hline \multirow{4}{*}{$\left(10^{20} / \mathrm{m}^{3}\right)$} & 0.6 & & & \\
\hline & 1.0 & 0.134 & .33 & 60 \\
\hline & 1.4 & 0.096 & .33 & $\underline{83}$ \\
\hline & 1.8 & 0.071 & .31 & 112 \\
\hline \multirow{4}{*}{$\begin{array}{c}T_{e o} \\
(k e V)\end{array}$} & 10 & 0.041 & .14 & 193 \\
\hline & 20 & 0.071 & .24 & 113 \\
\hline & $\underline{30}$ & 0.096 & .33 & 83 \\
\hline & 40 & 0.107 & .37 & 75 \\
\hline \multirow{4}{*}{$z_{\text {eff }}$} & 1.5 & 0.094 & .32 & 85 \\
\hline & 2.0 & 0.096 & .33 & 83 \\
\hline & 3.0 & 0.089 & .30 & 90 \\
\hline & 4.0 & 0.079 & .27 & 102 \\
\hline \multirow{4}{*}{$\begin{array}{l}E_{0} \\
\left(k e^{V}\right)\end{array}$} & 250 & 0.048 & .17 & 166 \\
\hline & 500 & 0.074 & .25 & 108 \\
\hline & $\underline{750}$ & 0.096 & .33 & 83 \\
\hline & 1000 & 0.105 & .36 & 76 \\
\hline \multirow{4}{*}{$\begin{array}{c}R_{\text {tang }} \\
(m)\end{array}$} & 3.6 & 0.066 & .23 & 121 \\
\hline & 3.9 & 0.085 & .29 & 94 \\
\hline & $\underline{4.2}$ & $\underline{0.096}$ & .33 & $\underline{83}$ \\
\hline & 4.5 & 0.098 & .34 & 82 \\
\hline \multirow{4}{*}{$\begin{array}{l}h_{b} \\
(m)\end{array}$} & 1.5 & 0.092 & .32 & 87 \\
\hline & 1.8 & 0.096 & .33 & 83 \\
\hline & 2.1 & 0.094 & .32 & 85 \\
\hline & 2.4 & 0.096 & .33 & 83 \\
\hline
\end{tabular}


Thus for NB current drive we can summarize as follows:

1. Present calculations yield $\gamma$ values competitive with wave drivers and in close agreement with earlier findings. Current profiles can be centrally peaked.

2. There appears to be an increase of $\gamma$ with $\bar{T}_{e}$, less than linear and similar to the HFFW scaling.

3. While $\gamma$ is maximized by utilizing $E_{b} \sim 3 \mathrm{MeV}$, it may be impractical to develop such high energy sources in the INTOR time frame.

\subsection{Ep itome of Steady State Calculations}

The four driver options are compared in Table IX. The LH driver is apparently the poorest performer, as it is hard to guarantee a centrally peaked current density at high $T_{e}(\sim 20 \mathrm{keV})$ and these moderate electron densities. The other three options are roughly comparable, with $y=0.33-$ 0.41 in the calculations. This implies that 68-84 MW of heating would suffice to achieve steady state operation with centrally peaked current profiles if INTOR were derated to operate at low power (low $\bar{n}_{e}$, per Table 1).

\section{CURRENT DENSITY PROFILE CONTROL}

\subsection{Value of Current Density Control}

Tokamak plasma stability and transport are influenced by details of the plasma, current density, and magnetic field spatial variations. For example, operation with $a_{a}$ sufficiently below unity is suspected to trigger the sawtooth behavior. As another example, operation at very high $B_{+}$and low current may be possible ${ }^{9}$ if hollow current profiles may be sustained. Noninductive current drive will prove valuable if it can maintain desired profiles indefinitely. 
Table IX

Summary of Steady State Benchmark Calculations

\begin{tabular}{|c|c|c|c|c|c|c|c|}
\hline Driver & $\left(10^{\bar{n}_{e}} \mathrm{~m}^{-3}\right)$ & $\begin{array}{l}\text { Io } \\
\text { (MA) }\end{array}$ & $\begin{array}{l}P_{C D} \\
(M W)\end{array}$ & $\gamma$ & $\begin{array}{l}\bar{T}_{e} \\
(k e V)\end{array}$ & $z_{e f f}$ & Notes \\
\hline LH & 0.68 & 7.9 & 87 & 0.30 & 4.8 & 1.5 & $\begin{array}{l}\text { Requires current diffu- } \\
\text { sion; higher T has } \\
\text { worse penetration. }\end{array}$ \\
\hline HFFW & 0.70 & 8.0 & 68 & 0.41 & 20 & 1.5 & $\begin{array}{l}0.3-1.0 \mathrm{GHz} ; 1.4<\mathrm{n}_{1} \\
<2.8 .\end{array}$ \\
\hline LFFW & 0.59 & 5.9 & 52 & 0.33 & 17 & 1.5 & $\begin{array}{l}64 \mathrm{MHz} ; 3.0 \leqslant n_{n}<4.5 \text {; } \\
\text { mode conversion not yet } \\
\text { studied. }\end{array}$ \\
\hline NB & 0.70 & 8.0 & 75 & 0.37 & 20 & 2.0 & $0.75 \mathrm{MeV}, \mathrm{D}^{\circ}$ \\
\hline
\end{tabular}




\subsection{LH Current Tailoring}

The LH driver may not provide ample flexibility to arbitrarlly shape the current profile. Indeed, we have already found that the LH waves do not penetrate into the magnetic axis region, so attaining centrally peaked profiles is very questionable. On the positive side there is ample theoretical evidence that surface currents may be driven with LH; see for example the analysis in Refs. 8, 9, and 11. In this sense LH current drive provides a current profile which cannot be maintained for long by inductive means. Thus the LH driver may be used, perhaps in combination with other drivers, to modify current density near the plasma edge.

\subsection{HFFW Current Tailoring}

In principle the HFFW offers considerable flexibility in controlling the current. Figure 25 shows results of HFFW calculations with a single mode (f $=$ $\left.0.4 \mathrm{GHz}, \mathrm{n}_{\|}=2.4, \mathrm{P}_{\mathrm{CD}}=50 \mathrm{MW}\right)$ launched into an INTOR sized plasma $\left(R_{0}=5.25\right.$ $m, k=1.6, A=6.0)$ at $\bar{T}_{e} \simeq 13 \mathrm{keV}$, with a parabolic density. As $n_{e o}$ and $\bar{n}_{e}$ are raised the pressure and $B_{t}$ increase. Also the wave damps further out in the periphery and the resulting current profile (insets in the figure) becomes hollow, as required for equilibrium at higher $\beta_{+}$. In Fig. 26 we observe that $j_{f}(R)$ may be adjusted by changing $f$. As found earlier, waves with lower $f$ have larger radial group velocities and damp closer to the magnetic axis. Hence, in the figure we find a more exaggerated current hollowing at the higher frequencies; this is associated with higher $B_{+}$equilibria.

An interesting possibility suggested by the results in Fig. 25 is that $\beta_{+}$ can be increased as $l_{0}$ is reduced. However, for conventional, inductively driven tokamaks the Troyon relationship would require $1_{0}$ to increase with maximum stable $\beta_{+}$. Likewise, the safety factor, $q(\psi)$, associated with the hollow $j_{+}(R)$ generated by a single mode spectrum may not be stable. The solution to this concern is the addition of multiple rays to the spectrum, in which case it is possible to select rays which duplicate a desired equillibrium with reasonable stability properties. This was exactly the procedure used in Sec. 2.3, where multi-mode spectra were used to create equilibria at the Trcion $B_{t}$ limit with $q(\psi)$ varying monotonically from $q_{a}=1.0$ to $q_{b}=2.0$. Thus, for example, the necessary $j_{f}(R)$ profiles given in the insets of Fig. 12 are created for different $B+$ values by adjusting the power spectrum. 


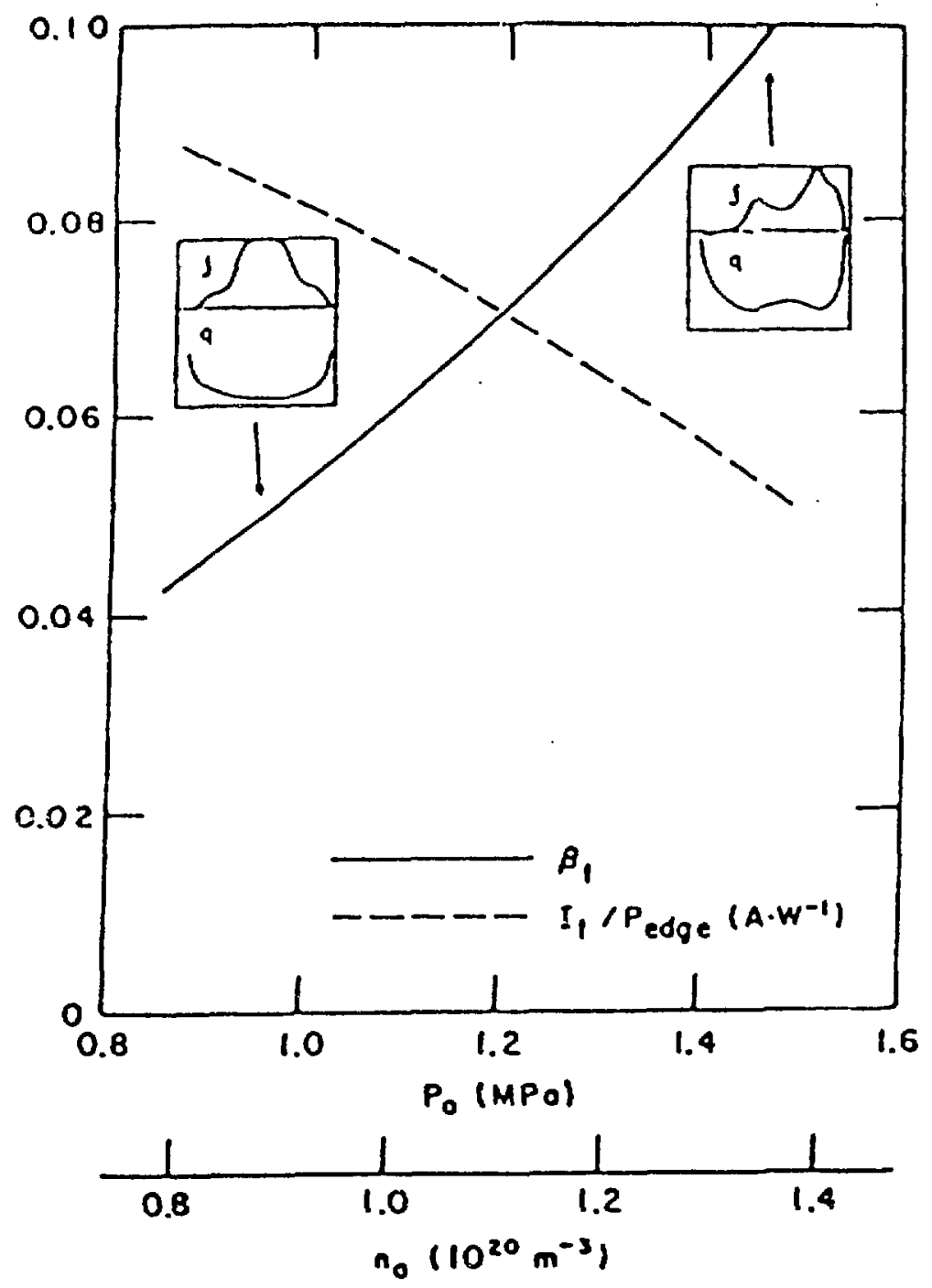

Figure 25: Equilibrium beta and toroidal current for various peak densities and pressures; all other parameters are those of the reference case, ${ }^{*}$ except $T_{D O}=42.9 \mathrm{kev}$. Toroidal current density $j$ and safety factor $q$, shown in the insets, vary along the curves.

$$
\begin{aligned}
* R_{0} & =5.25 \mathrm{~m}, A=6, k=1.6, B_{0}=3.7 \mathrm{~T}, T_{e O}=18 \mathrm{keV}, \alpha_{n}=1.05, \\
\alpha_{T} & =0.35, P_{C D}=50 \mathrm{MH}, f=0.4 \mathrm{GHz}, n_{1}=2.4 .
\end{aligned}
$$




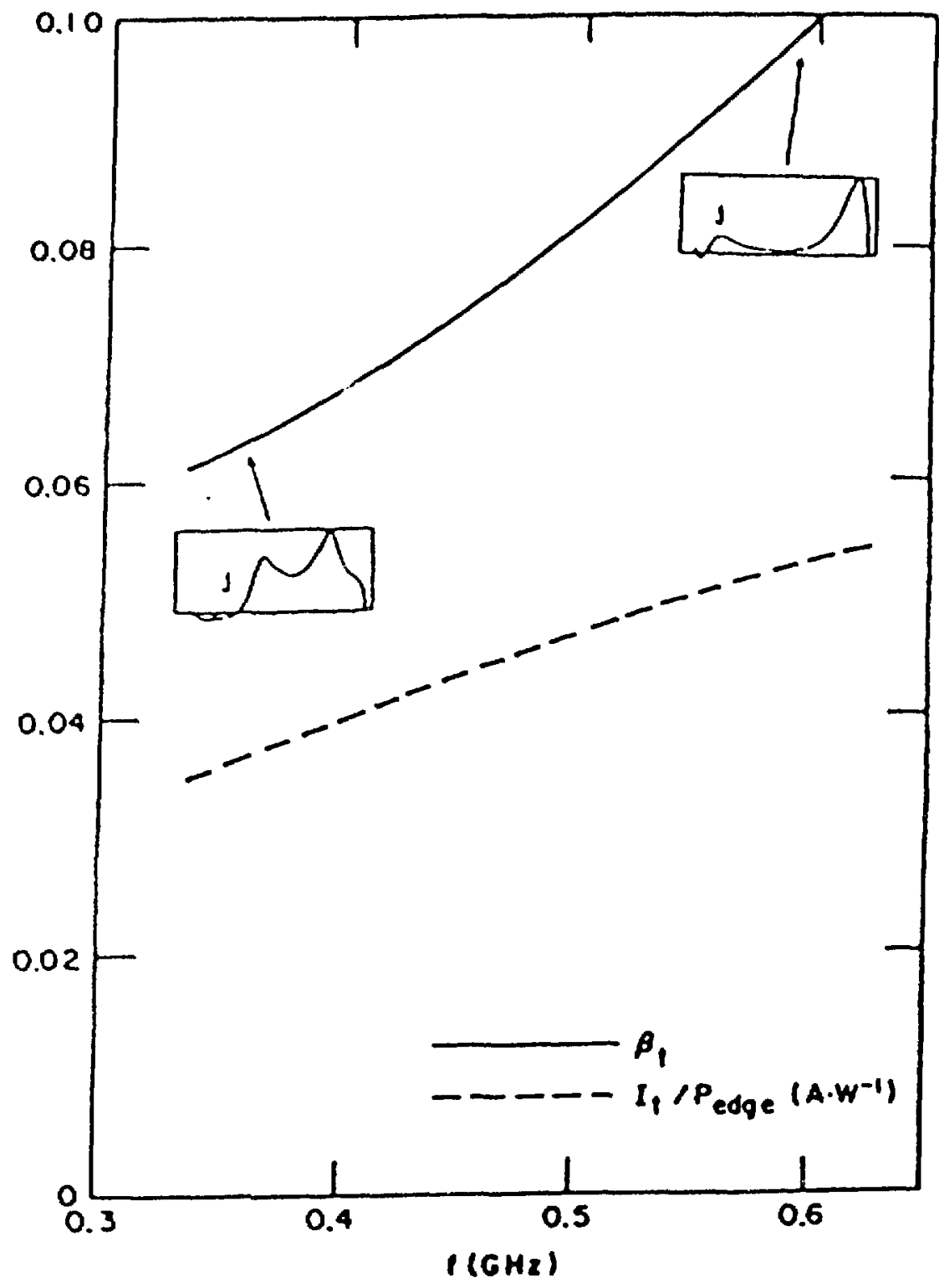

Figure 26: Equilibrium beta and toroidal current for various fast wave frequencies; all other parameters are those of the reference case,* except $T_{\text {eo }}=15.0 \mathrm{keV}, T_{D O}=15.5 \mathrm{keV}$ and $P_{\text {edge }}=60 \mathrm{MW}$. The toroidal current density is shown in the insets.

See Fig. 25 . 
On the other hand, it is hoped that eventually the limitations of Troyon may be breached; ${ }^{l}$ an example of high $\beta_{+}$, low $1_{0}$, with a monotonic $q(\psi)$ is given in Figs. 27-30. This example is for an INTOR-class tokamak with $R_{0}=$ 5.2 $\mathrm{c}, \mathrm{m}, \mathrm{A}=6.0, k=1.6, d=0.5, \bar{n}_{e}=0.98 \times 10^{20} \mathrm{~m}^{-3}$, and $\bar{T}_{e}=13 \mathrm{keV} . \mathrm{A}$ comination of LH and HFFW modes are used:

\begin{tabular}{cccc} 
Polarization & Frequency $(\mathrm{GHz})$ & & Edge Power (MW) \\
\cline { 2 - 2 } & 1.5 & 2.8 & 0.22 \\
LH & 1.5 & 2.2 & 0.90 \\
LH & 1.5 & 1.9 & 0.36 \\
FW & 0.6 & 2.6 & 27.0 \\
FW & 0.6 & 1.8 & 5.0 \\
FW & 0.3 & 2.0 & 3.0 \\
FW & 0.3 & 1.8 & 3.5 \\
FH & 0.3 & 1.7 & 5.0 \\
FW & 0.3 & 1.6 & 1.5
\end{tabular}

The converged equilibrium has $B_{+}=0.079, l_{0}=3.2 \mathrm{MA}, q_{a}=0.98, q_{b}=1.95$, $\varepsilon B_{p}=0.7$, and requires $P_{C D}=46.5 \mathrm{MW}$. We see $B_{+}$is nearly twice the Troyon value: $\quad B_{T}=0.04(3.2 / 0.875 / 3.66)=0.040$. The pressure and $\psi$ contours in the figures display a mild Shatranov shift (the magnetic axis is at $5.54 \mathrm{~m}$ ). However, the hollow current is very desirable for noninductive current drive; although in this example $\gamma$ is only $\sim 0.35$ the low total $I_{0}$ at this $B_{f}$ reduces $P_{C D}$ to quite reasonable values. Such low lo would also ease the design (reduce the cost) of the poloidal field coil set for INTOR.

\subsection{LFFH Current Tailoring}

Profile control with the LFFW has not yet received much study. However, as discussed in Sec. 2.4, profile control may require off-midplane launchers

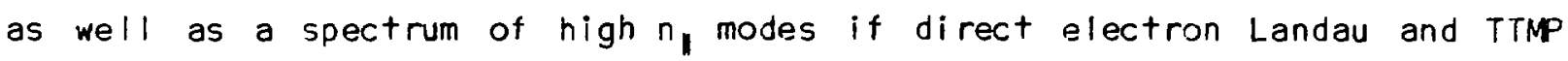
damping is desired. Mode conversion or minority heating current drive are highly spatially dependent, so we would expect to readily achieve control with these alternative techniques. 

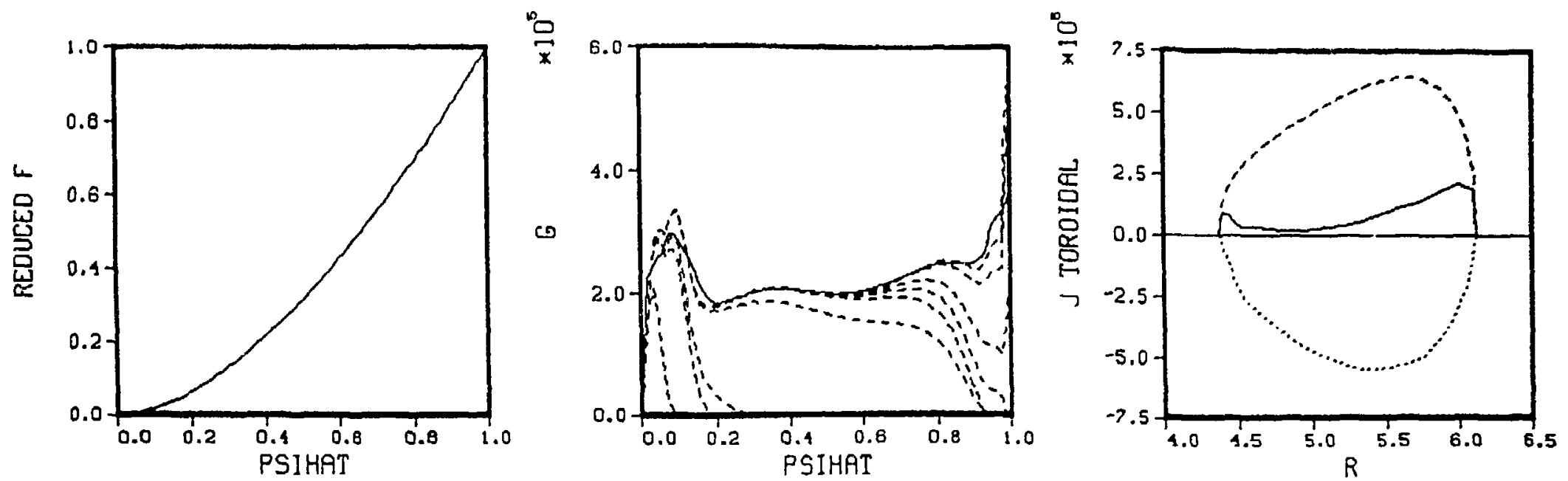

@)
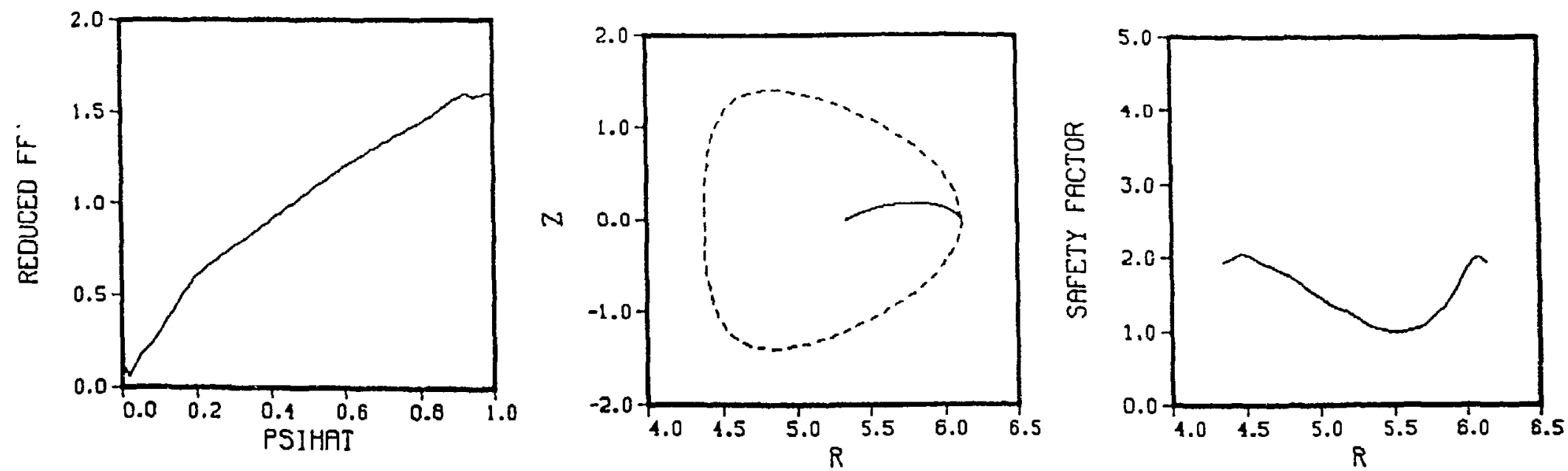

Figure 27: High beta equilibrium generated with the HFFW (plus a small amount of LH power to control the edge current density): $B_{t}=0.079, \mathrm{l}_{0}=3.2 \mathrm{MA}, \mathrm{B}_{0}=3.66, \mathrm{R}_{0}=5.25$ $\mathrm{m}, \bar{n}_{e}=0.98 \times 10^{20} \mathrm{~m}^{-3}, \bar{T}_{e}=13 \mathrm{keV}$, and $\gamma=0.35 ; P_{C D}=47 \mathrm{MW}$. 
PSI

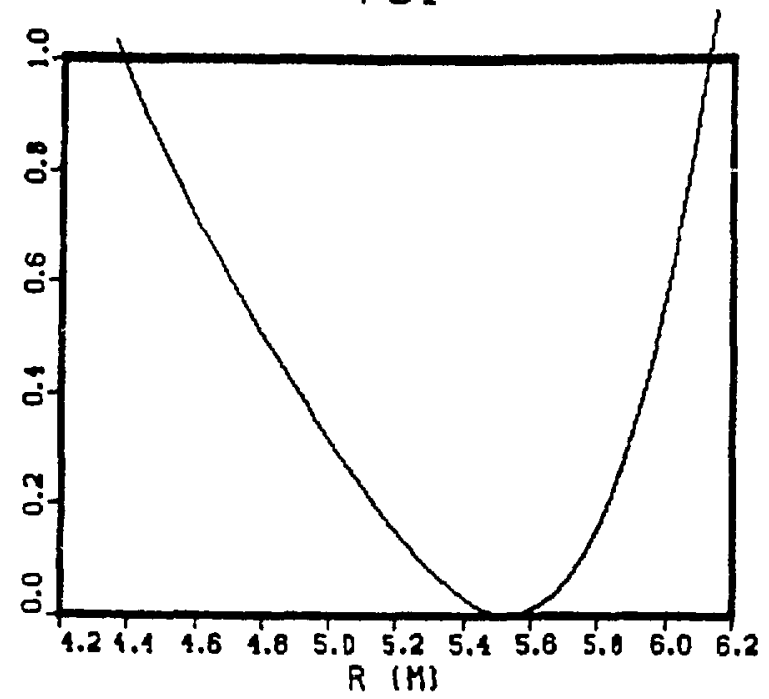

J TOROIDAL

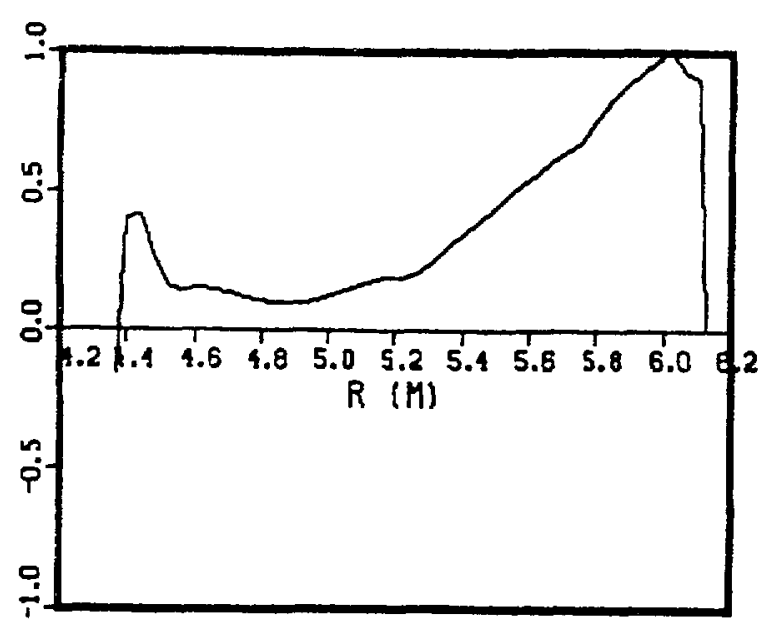

PRESSURE

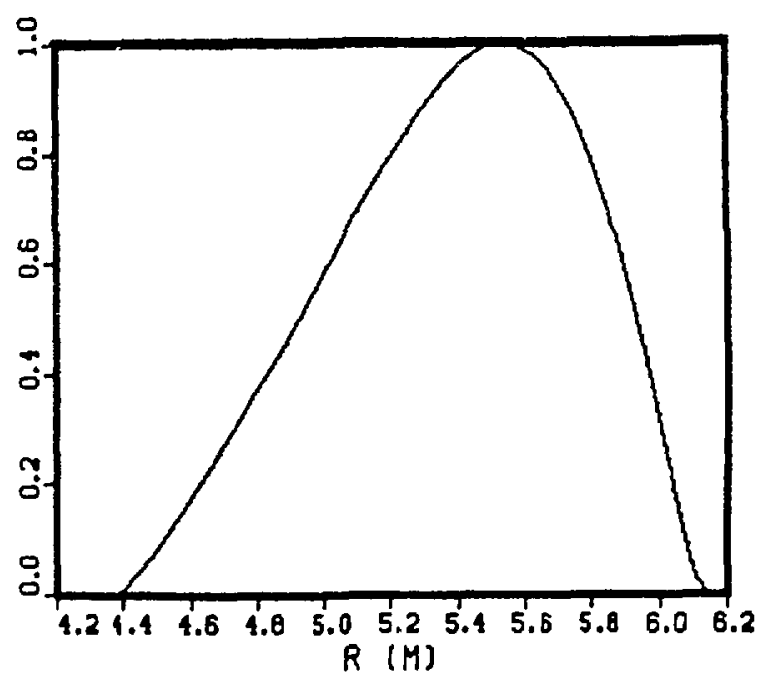

0

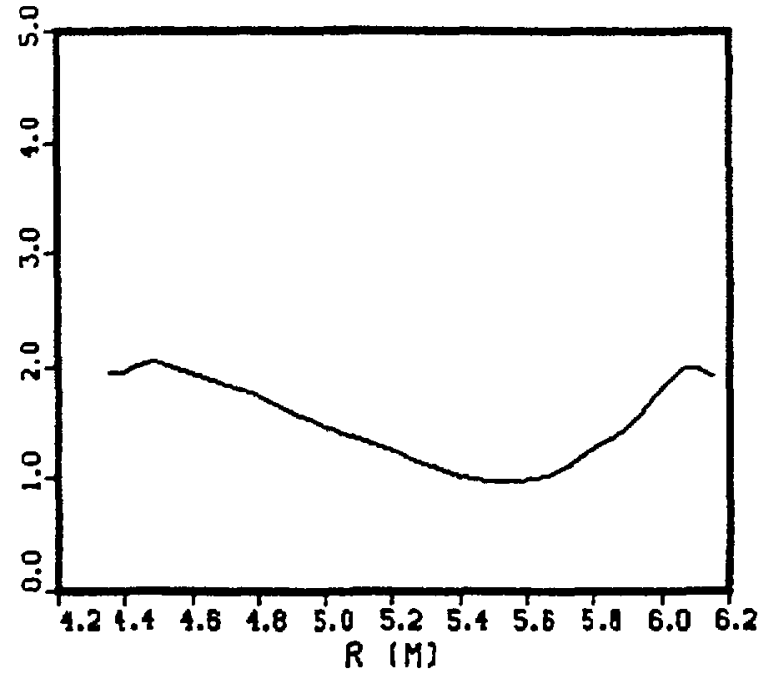

Figure 28: Pressure and poloidal flux across midplane for Fig. 27. 
CURRENT DENSITY CONTOURS

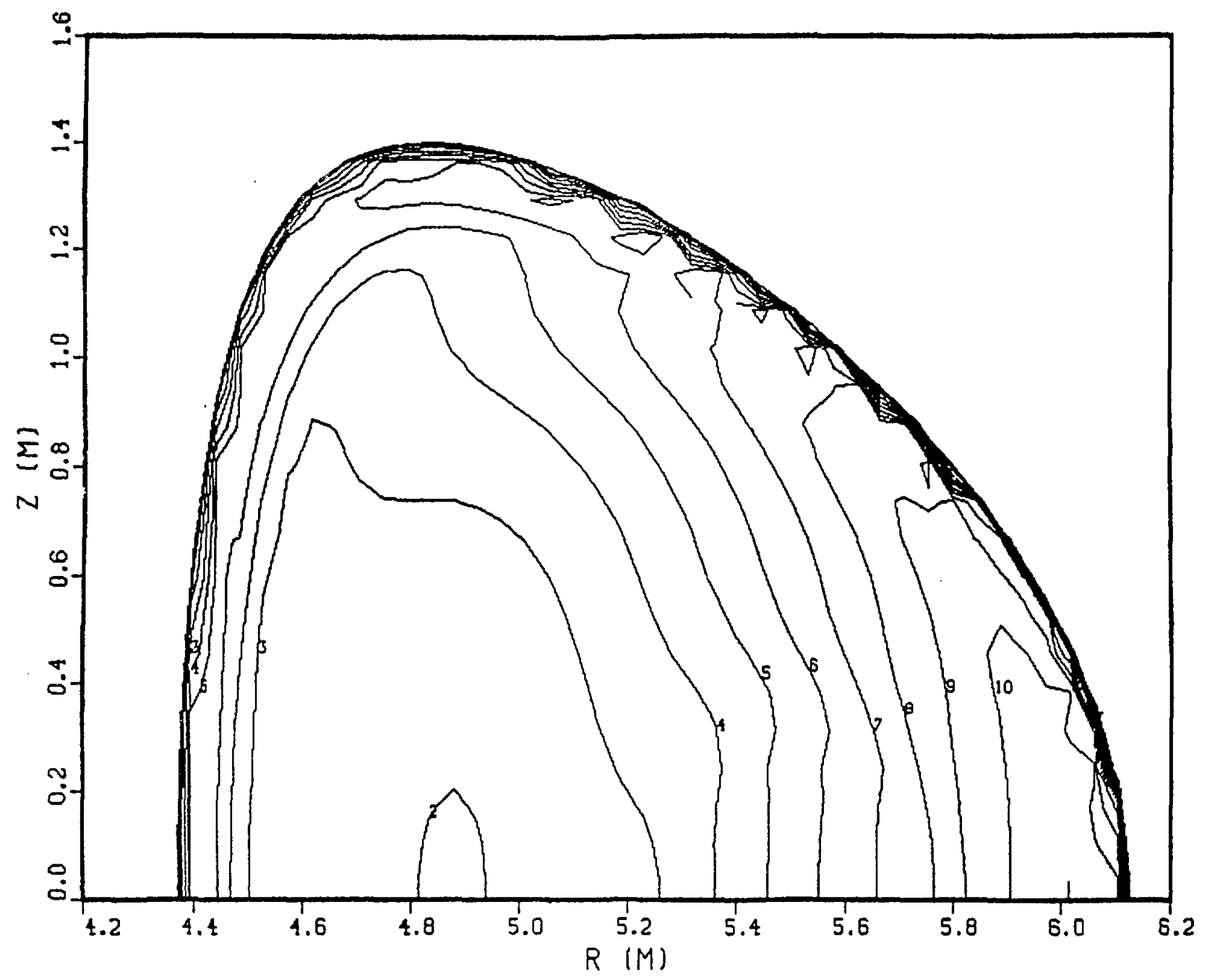

Figure 29: Hollow current density profile for Fig. 27. 
PSI CONTOURS

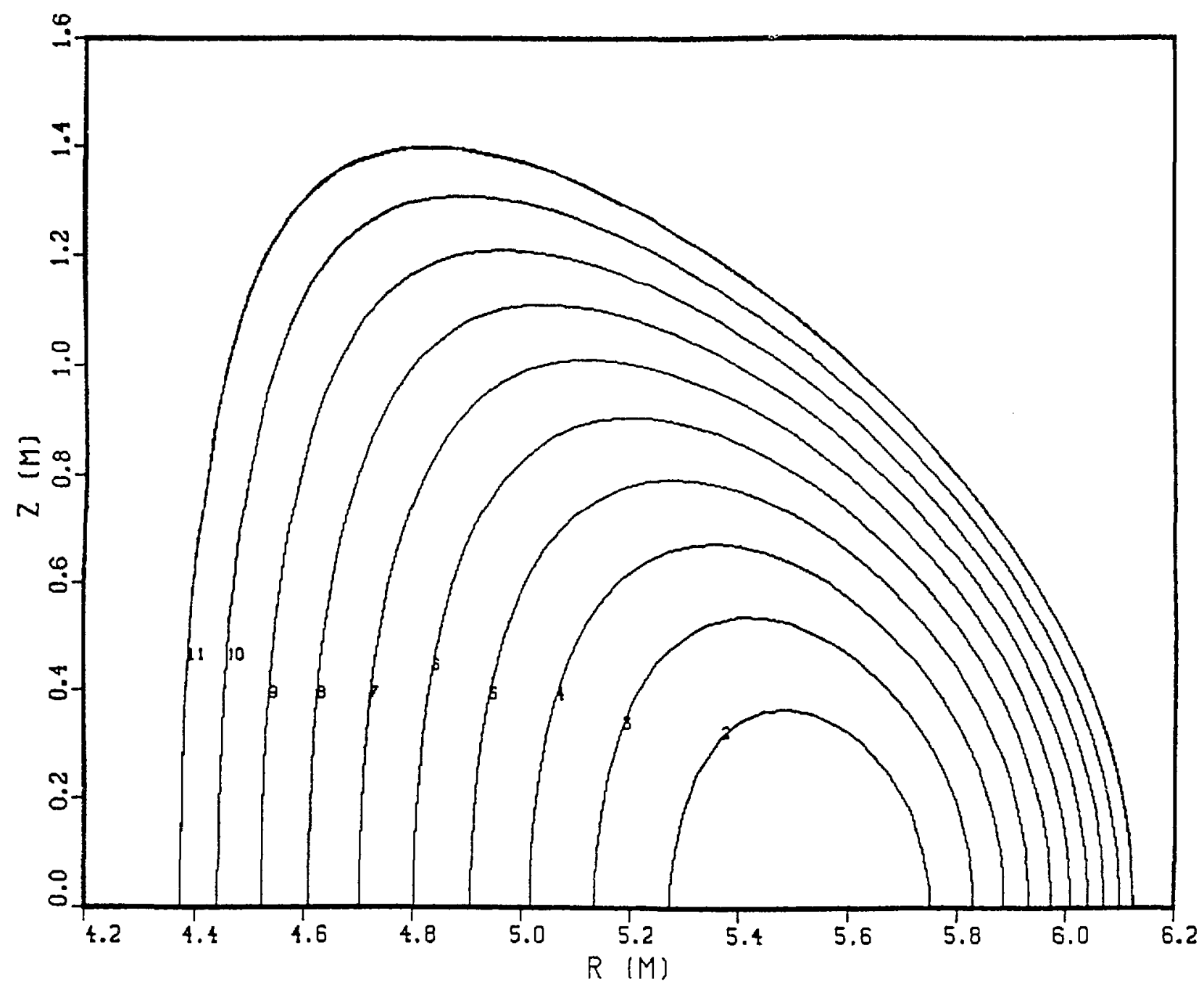

Figure 30: Poloidal flux distribution for Fig. 27. 


\subsection{B Current Tailoring}

A high degree of current profile control is posslble, in principle, because there are no accessibility conditions which would prevent neutral beam current drive in any region of the plasma. The current profile could be varied during a burn by turning individual beamlets on and off. It should be possible to generate current protiles which are very centrally peaked, very broad, hollow, or even localized around a glven minor radius far from the magnetic axis. The only limiting factor would be the low current drive efficiency in the cold outer regions of the plasma.

In our studies of current profile control the source size is reduced to a point and the beam intensity in its cross section is determined by a Gaussian function. The beam centerline is parallel to and located a distance $Z_{b}$ below the tokamak equatorial plane. This produces deposition (and current drive) predominantly in a toroidal "shell" at a minor radius which is tangent to the neutral beam.

The current profile produced by the neutral beam geometries studied in Sec. 2.5 was relatively broad with $q(r=a) \sim 2 q(r=0)$ and $q(r)$ roughly flat for $r<a / 2$. If a large current is spontaneously generated by the neoclassical "bootstrap" effect it would be more desirable to drive a centrally peaked "seed" current. This is easily done by reducing the beam source height, $h_{b}$, and moving $R_{\text {tang }}$ closer to $R_{0}$. It is also possible to tailor a broad driven current profile in detail by controlling the current of individual beamiets since the negative ion beam is made of many parallel beamlets drawn from many independent sources. The granularity scale of this local control is determined by the divergence of a beamlet and is in the range of $10-30 \mathrm{~cm}$. By injecting along a path which is parallel to but below the tokamak equatorial plane a distance $Z_{b}$, it is possible to concentrate the deposition -- and the driven current -- in a relatively narrow radial "shell". The expansion of the flux surfaces in a vertically elongated equilibrium effectively reduces the beam size and produces a narrow region of current of radial extent $\Delta r$ (full width at half maximum is given in Table $X$ ). The current drive efficiency is not a strong function of $r$ - with the assumed density and temperatur profiles -- but it is expected to drop in the colder plasma periphery. 
Table $X$

Shell Currents with NB

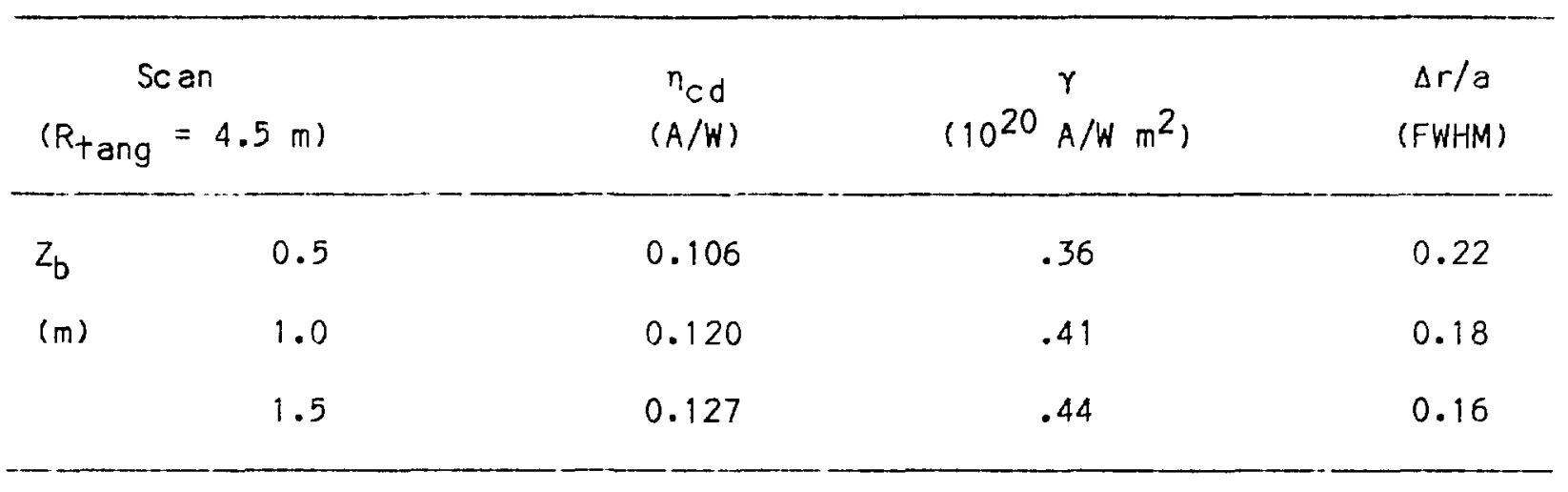


The principle limitation on current profile control with neutral beam current drive may be the physical limitation imposed by the size and spacing of the toroidal field colls on the aiming of the neutral beams. It may be that the TF coils will need to be redesigned to accommodate the neutral beams in order to permit the optimum orientation, $R_{0}-a / 2<R_{\text {tang }}<R_{0}$.

lllustrations of various current profiles for INTOR are shown in Fig. 31. We note that Ref. 21 also reports that current profiles may be controlled, although their approach, which involves a variety of $R_{\text {tang }}$ values, may be more difficult to implement than our scheme, which involves vertical variation of the beam source density.

\section{CURRENT DRIVE WITH REVERSED ELECTRIC FIELD}

\subsection{Theoretical Model for Wave-Driven Currents}

Based on the presentations and discussions at the INTOR-Related Specialists' Workshop on Noninductive Current Drive, IPP-Garching, September 15-17, 1986, Ref. 4., there appears to be a consensus that the Karney-Fisch theory 23 is a good model which reproduces the features of current ramp-up and transformer recharging seen in various experiments. For the purposes of studies on INTOR we can provide the following simplified algorithms to model current drive when $E \neq 0$.

The total toroidal current in the plasma is

$$
1=\left(v_{\ell} / R_{S_{P}}\right)+I_{C D}
$$

where the loop voltage is $V_{\ell}=2 \pi R_{0} E$ and $R_{S_{P}}$ is the Spitzer toroidal resistance. (Presumably a more detailed theory would modify $R_{S_{P}}$ to include neoclassical effects.) The noninductive current, $I_{C D}$, is related to the value where $E=O$ by the following approximate formula:

$$
I_{C D}=\gamma\left(\frac{P_{C D}}{\bar{n}_{e} R_{0}}\right) \times N\left(u_{r}\right) .
$$


INTOR: $\mathrm{h}_{\text {beam }}=1.5,1.8,2.1,2.4 \mathrm{~m}$

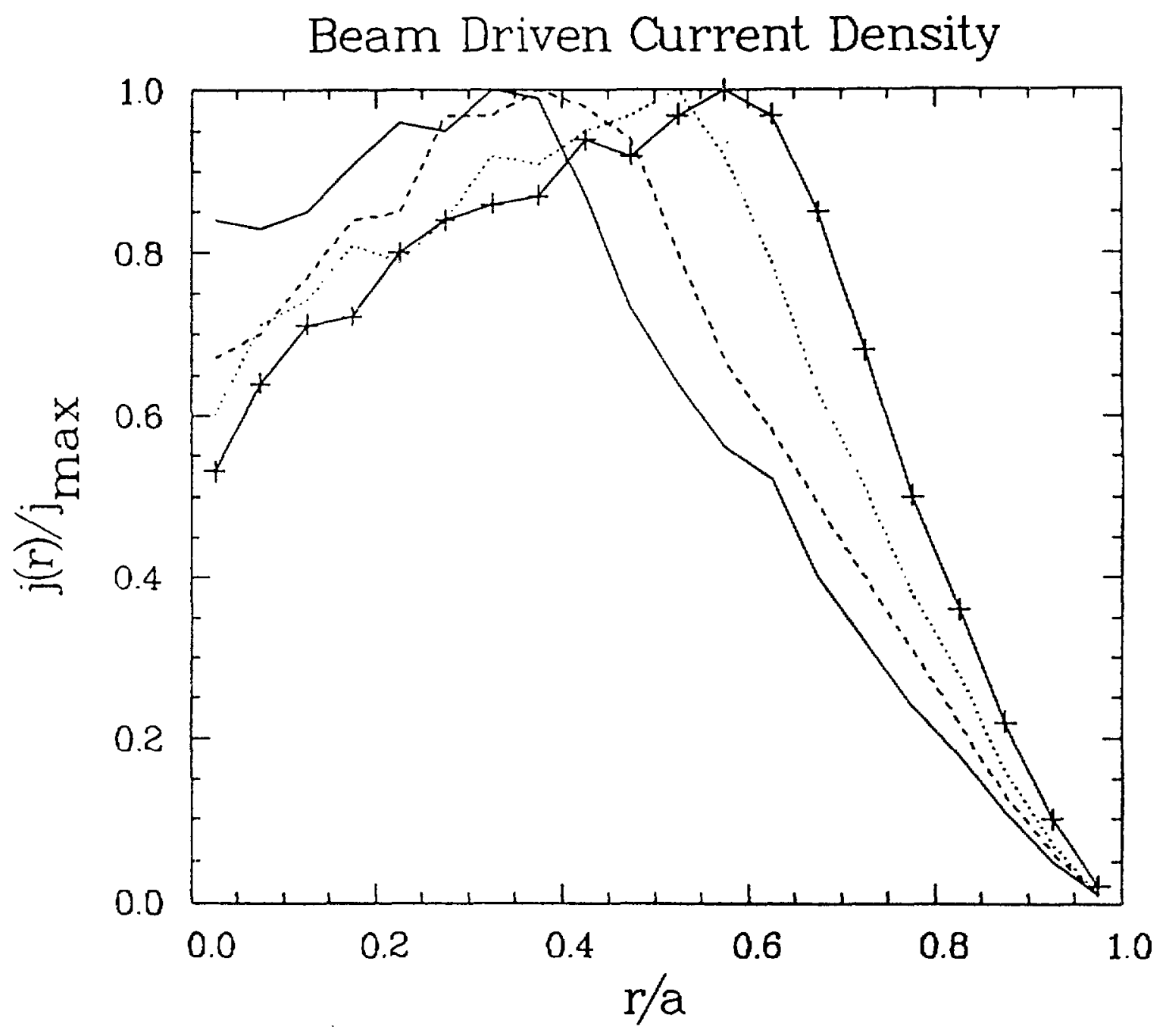

Figure 31a: Current density profiles for neutral beam driven current with extended source of varying height above the midplane. 
INTOR: $\mathrm{Z}_{\text {beam }}=0.5,1.0,1.5 \mathrm{~m}$

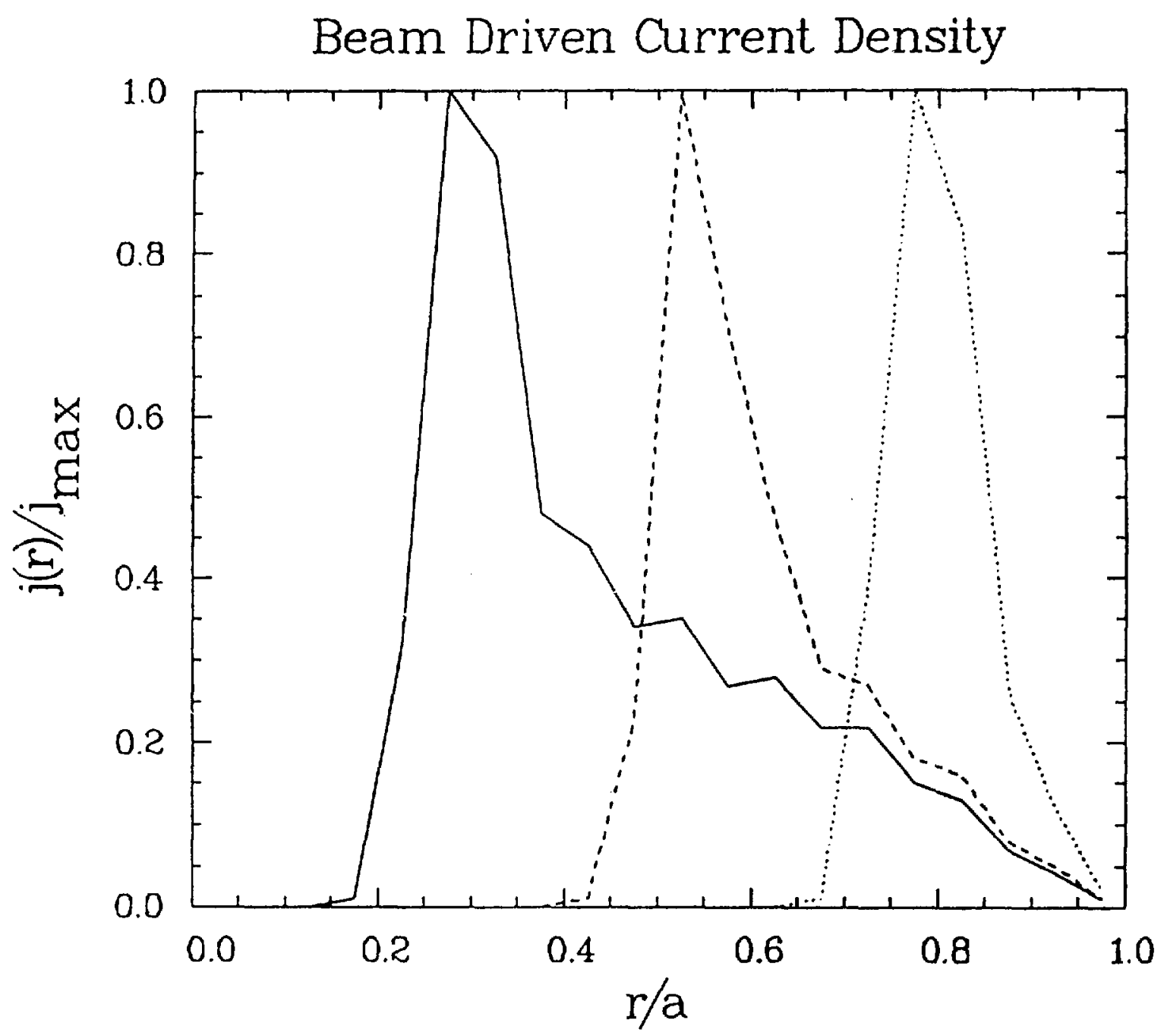

Figure 31b: Current density profile with localized neutral beam source at different vertical positions. 
Here $y P_{C D} / \bar{n}_{e} R_{0}$ is the current driven by an absorbed power, $P_{C D}$, when $E=0$. The factor $N$ enhances the noninductive current with a forward emf $\left(u_{r}<0\right)$, such as occurs during current ramp-down, and $N$ reduces the current with a reverse emf $\left(u_{r}>0\right)$, during ramp-up. The argument of $N\left(u_{r}\right)$ is the ratio of wave phase speed to the runaway speed,

$$
u_{r}=c / n_{n} / v_{r}
$$

where $n_{\|}$includes any upshift in the plasma. The runaway speed, in MKS units, is given by

$$
v_{r}=9.33 \times 10^{-3} \sqrt{n_{e}}|\bar{E}|
$$

The function $N$ is displayed in Fig. 32 for $Z_{\text {eff }}=5$. For Eq. (7) to be valid there must be no generation or retention of runaway electrons, which implies the following constraint:

$$
-1<u_{r}<1.5
$$

Since ur varies spatially throughout the plasma, it is problematic how to evaluate $u_{r}$ or $N$ in a zero-dimensional calculation. One possibility is to crudely evaluate $u_{r}$ by using $n_{\|}=2.5$, putting $n_{e}$ equal to the average value, $\bar{n}_{e}$, setting $E$ to the value given at the major radius $R_{0}$.

The function $N$ is only mildly dependent on $Z_{\text {eff, but one should recail }}$ that $\gamma$ has a $Z$ eff dependence. It should be emphasized that the Karney-Fisch theory assumes waves at high phase speeds, $c / n_{n} / v_{e} \gg 1$, where $v_{e}$ is the electron thermal speed, and this constraint is usually satisfied at low $T_{e}$ $(\leqslant 2 \mathrm{keV})$. If somehow slower phase speeds were used $\left(\mathrm{c} / \mathrm{n}_{n} / \mathrm{v}_{\mathrm{e}} \simeq 1\right)$ a different formulation of the electric field effects would be needed, as, for example, given by Raf. 24 .

\section{2 내 Current Drive}

We evaluate first the inductive term in Eq. (6). From Table $1: v_{\ell}=$ $2 \pi R_{0} E=0.308 \mathrm{~V}$. We use a rough formula for the neoclassical resistance, viz. $R_{S_{P}}=1.02 \times 10^{-7} \Omega \times\left(Z_{e f f} / 1.5\right)\left(2 \mathrm{keV} / \bar{T}_{e}\right)^{3 / 2}$. Hence, for $1=8.0 \mathrm{MA}, \overline{\mathrm{T}}_{\mathrm{e}}=2$ 


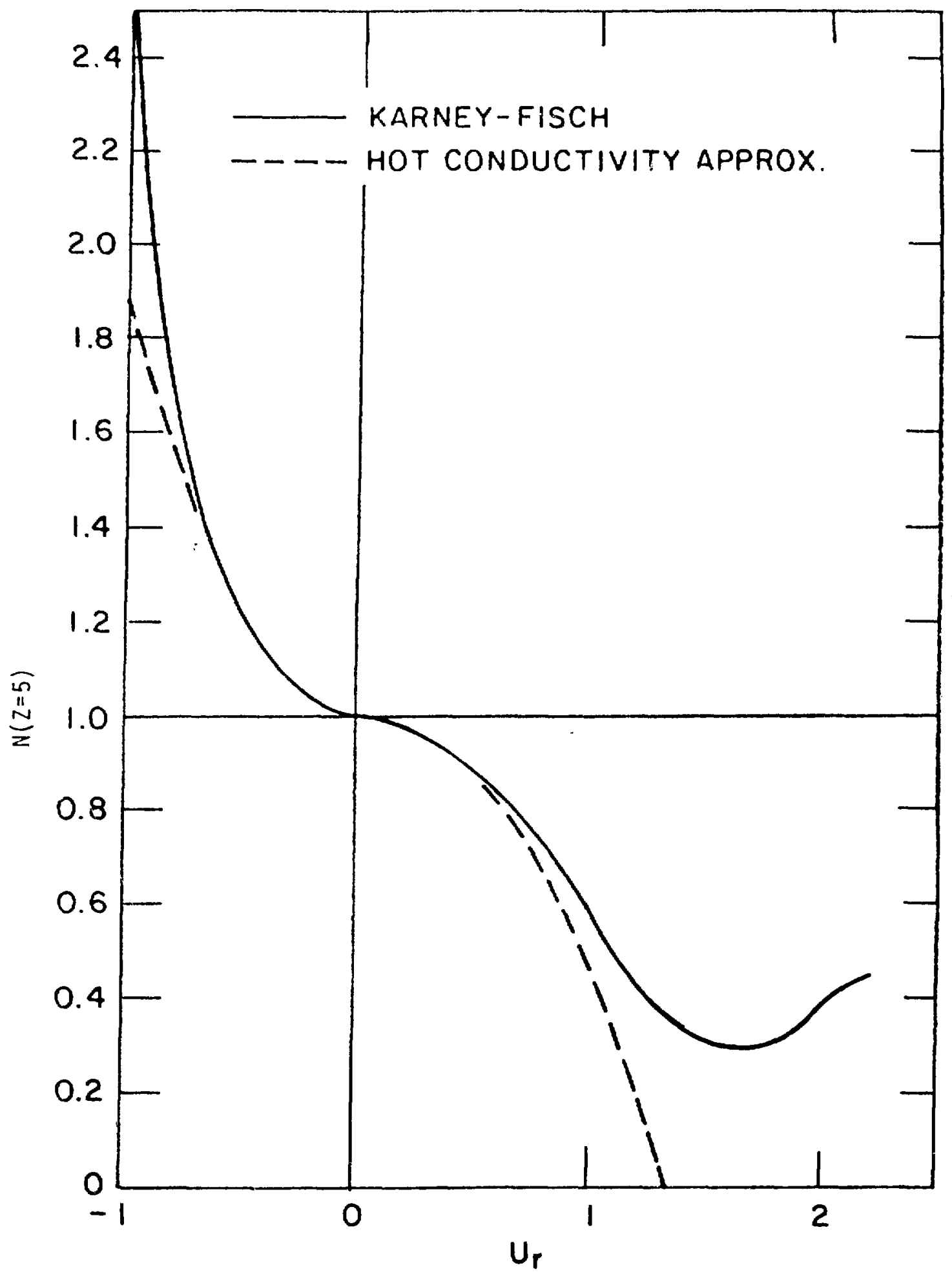

Figure 32: Factor multiplying $\gamma$ when $E \neq 0$. Normalized phase speed to runaway speed is $u_{r} \equiv c /\left(n_{n} v_{r}\right)$, where $v_{r} \equiv 9.3 \times 10^{-3} \sqrt{n_{\mathrm{e}} / E}$ in sI units. During ramp-up the sign convention is $u_{r}>0$. 
keV, and $Z_{\text {eff }}=1.5$, we require $I_{C D}=8.0 \mathrm{MA}+3.0 \mathrm{MA}=11.0 \mathrm{MA}$. Thus the $\mathrm{LH}$ waves must considerably overdrive the current in the face of the $E=-0.01 \mathrm{~V} / \mathrm{m}$ ent.

Now we evaluate $r$ in Eq. (7) for the low $\bar{n}_{e}$ and $\bar{T}_{e}$ conditions in Table 1. This low density and temperature correspond to $B_{t}=0.00021$, and we seek an LH generated equilibrium at this beta with a current of $11 \mathrm{MA}$. A solution is found (see Fig. 33) with a single $f=5.0 \mathrm{GHz}$ and a total power of $P_{C D}=$ $20.6 \mathrm{MW}$ spread over $2.4<\mathrm{n}_{n}<10.0$. The value (at $\bar{n}_{\mathrm{e}}=0.039 \times 10^{20} \mathrm{~m}^{-3^{2}}$ and $\left.\bar{T}_{e}=2.0 \mathrm{keV}\right)$ of $\gamma$ is 0.10 , and we note in Fig. 33 that there is no problem attaining centrally damped $L H$ waves at these very low $\bar{n}_{e}$ and $\bar{T}_{e}$ values. Referring to Fig. 1 we find $y=0.10$ is quite conservative and $f$ alls below the values experimentally obtalned under these conditions. 12

Next, we must evaluate $u_{r}$ in $E q$. (8) to learn if reverse runaways are a concern. Each ray in $G(\tilde{\psi})$ in Fig. 33 damps closer to the magnetic axis as $n_{\text {, }}$ decreases. We evaluate $u_{r}$ from the local $n_{e}$ value where each $n_{\text {, value has }}$ maximum damping and $f$ ind that $u_{r}$ increases from 0.37 at the boundary to 0.51 near the axis. Thus, from Fig. 32, we find $N=0.9$ throughout the plasma volume for this case with $E=-0.01 \mathrm{~V} / \mathrm{m}$. Not only is the runaway probability zero, 23 but the hot conductivity formula, 25 represented by the dashed line in Fig. 32, could be used to model the electric field effects.

Finally, from Eq. (7) we find the requisite $L H$ power for this mode of operation is $P_{C D}=\bar{n}_{e} R_{O}{ }_{C O} /(Y N)=24 \mathrm{MW}$. It is not evident what combination of parameters would actually minimize $P_{C D}$. For example, higher $Z_{\text {eff }}$ would reduce the return current and hence the required ICD; however, higher $Z_{\text {eff }}$ would also reduce $Y$ somewhat. Likewise, $\bar{T}_{e}=2 \mathrm{keV}$ might be too low if $P_{C D}=$ $24 \mathrm{MW}$ is heating such a low density plasma. The sensitivity of $P_{C D}$ to these parameters may be found straightforwardly in the manner just outlined.

\subsection{Fast Wave Current Drive}

Calculations of $\gamma$ at $\bar{T}_{e}=2 \mathrm{keV}$ have not yet been performed for the HFFW and LFFW. However it is likely that $y=0.10$ could be expected, so the result for $P_{C D}$ would be roughly the same as tound in Sec. 4.2 for the LH driver. 

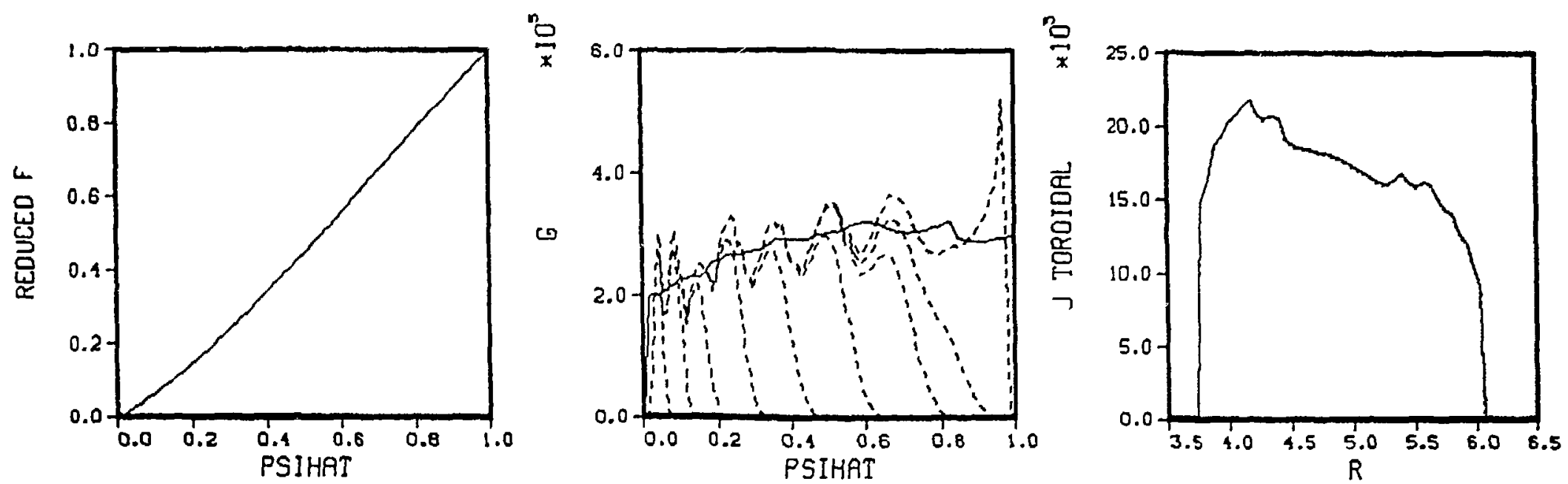

जั
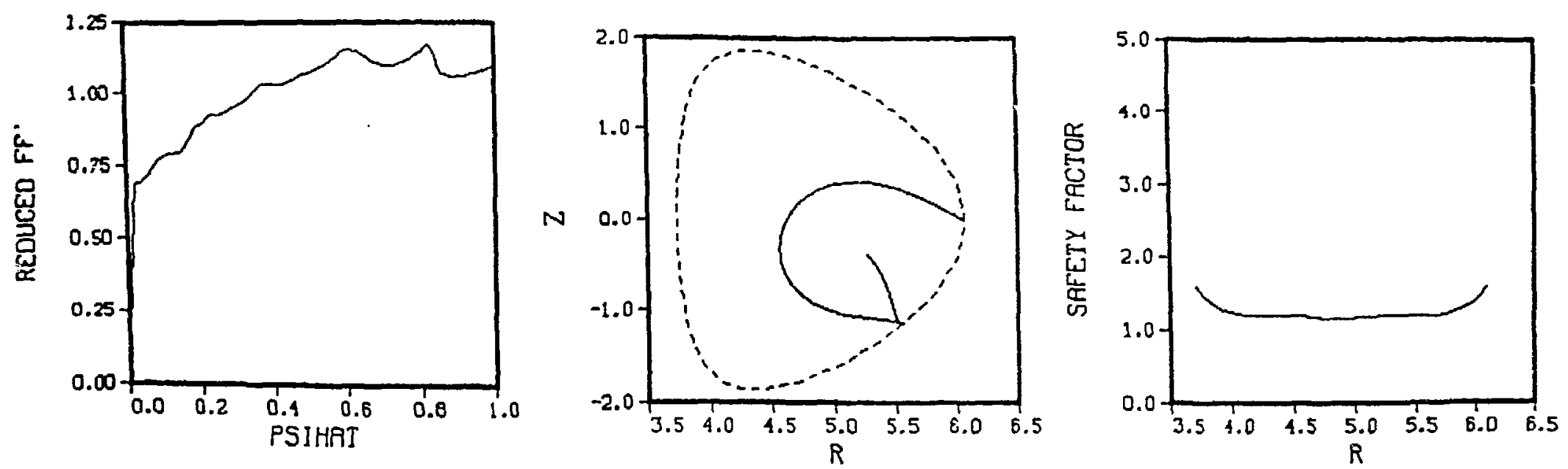

Figure 33: Benchmark INTOR for very low $\bar{n}_{e}\left(=0.04 \times 10^{20} \mathrm{~m}^{-3}\right)$ transformer recharge with LH waves: $T_{e}=2 \mathrm{keV}, \gamma=0.10$, and centrally peaked current is obtained with $f=5.0$ $\mathrm{GHz}$ and $2.4 \leq n_{\|} \leq 10.0$. 


\subsection{MB Current Drive}

For the benchmark calculation in Table 1 we took $Z_{\text {eff }}=9$ for the NB. Hence the return current is one sixth the value obtained for the LH driver (with $\left.Z_{\text {eff }}=1.5\right): I_{C D}=8.5 \mathrm{MA}$. The benchmark parameters are listed in Table VII, and the results are listed in boldface in Table XI.

The benchmark case has a low $\gamma=0.037$ and high $P_{C D}=45 \mathrm{MW}$. A more attractive reference case (underlined in Table $X 1$ ) was selected for a sensitivity study; for the reference we note $P_{C D}=26 \mathrm{MW}$, which is comparable to the LH driver. Increasing the density allows $\gamma$ to increase because the shinethrough is reduced at higher density and more of the injected power drives current. The $\gamma$ dependence is almost linear foi $T_{\text {eo }}<10 \mathrm{keV}$, with saturation at higher temperatures. Note that the required beam power is actually minimized for $T_{e o} \sim 12 \mathrm{keV}$ (even though $\gamma$ increases monotonically) because $I_{O H}$ rises as $T_{e o}{ }^{1.5}$. The optimum $z_{\text {eff }}$ for $\gamma$ is $\sim 6$, while for $P_{C D} i t$ is $\sim 9$. At higher density the shinethrough would be reduced and the optimum $Z_{\text {eff would be lower. }} P_{C D} f$ alls and $\gamma$ increases with increasing beam voltage in spite of the higher shinethrough. At higher density the higher beam voltages would be even more advantageous.

The effect of a reversed emf on NB driven currents has not received a lot of attention. We have not considered three normally ignorable fast-ion slowing-down effects which may be non-negligible in the transtormer recharge scenario:

1. The reversed electric field slows down the comgoing fast ions at a rate which is comparable to that due to collisions with the background plasma. At $T_{e} \sim 2 \mathrm{keV}$ this is only a $10 \%$ effect, but it grows to $50 \%$ at $T_{e} \sim 6 \mathrm{keV}$ (for $500 \mathrm{keV}$ deuterons at the benchmark density).

2. The drift velocity of the electrons due to the reversed electric field could be as high as 5-10\% of the speed of a $500 \mathrm{keV}$ deuteron. The relative speed between the co-going fasi ions and the electrons will therefore be decreased and the rate of $f$ ast ion energy loss to electrons could be decreased by up to $25 \%$. (The calculations assume 
Tab le XI

NB Current Drive with Reversed Electric Field

\begin{tabular}{|c|c|c|c|c|c|c|}
\hline \multicolumn{2}{|l|}{ Scan } & \multirow{2}{*}{$\begin{array}{c}\eta_{C d} \\
(A / W) \\
0.418\end{array}$} & \multirow{2}{*}{$\begin{array}{l}Y \\
\left.\mathrm{~A} / \mathrm{W} \mathrm{m} \mathrm{m}^{2}\right)\end{array}$} & \multirow{2}{*}{$\frac{\begin{array}{c}P_{b} \\
(M W)\end{array}}{25}$} & \multirow{2}{*}{$\begin{array}{l}\begin{array}{l}1 O H \\
(M A)\end{array} \\
2.3\end{array}$} & \multirow{2}{*}{$\begin{array}{c}\text { f shing: } \\
0.37\end{array}$} \\
\hline & 6 & & & & & \\
\hline \multirow{4}{*}{$\left(10^{13} / m^{3}\right)$} & 3 & 0.391 & .077 & 26 & 2.3 & 0.27 \\
\hline & 10 & 0.338 & .083 & 30 & 2.3 & 0.20 \\
\hline & 12 & 0.307 & .090 & 34 & 2.3 & 0.14 \\
\hline & 4 & 0.188 & .037 & 45 & 0.5 & 0.26 \\
\hline$r_{\text {eo }}$ & 8 & 0.307 & .060 & 30 & 1.3 & 0.27 \\
\hline \multirow[t]{3}{*}{$(k e v)$} & 12 & 0.391 & .077 & 26 & 2.3 & 0.27 \\
\hline & 16 & 0.445 & .087 & 26 & 3.5 & 0.27 \\
\hline & 3 & 0.378 & .074 & 36 & 5.7 & 0.48 \\
\hline \multirow[t]{4}{*}{$Z_{\text {eff }}$} & 6 & 0.402 & .079 & 28 & 3.3 & 0.36 \\
\hline & $\underline{9}$ & 0.391 & .077 & 26 & 2.3 & 0.27 \\
\hline & 12 & 0.354 & .069 & 28 & 1.8 & 0.20 \\
\hline & 200 & 0.263 & .052 & 39 & 2.3 & 0.20 \\
\hline$E_{0}$ & 300 & 0.338 & .066 & 30 & 2.3 & 0.24 \\
\hline \multirow[t]{2}{*}{ (kev) } & 400 & 0.391 & .077 & 26 & 2.3 & 0.27 \\
\hline & 500 & 0.434 & .085 & 24 & 2.3 & 0.30 \\
\hline
\end{tabular}


that the background $p$ lasma is at rest in the lab frame.)

3. Any plasma rotation caused by the beams will tend to reduce the relative speed between the co-going fast ions and the electrons also. (The corresponding increase in the fast-ion drag due to thermal ions remains negligible since ion drag is small compared to electron drag.)

These effects offset each other to some extent; future work should probably include them in the fast-ion slowing-down model.

In summary, in the benchmark plasma corresponding to the $\mathrm{OH}$ transformer recharge scenario, a current of $8.5 \mathrm{MA}$ ( $8 \mathrm{MA}+0.5 \mathrm{MA}$ to offset the current driven by the reversed electric field of $0.01 \mathrm{~V} / \mathrm{m}$ ) can be driven by $45 \mathrm{MW}$ of $400 \mathrm{keV}$ neutral deuterium beams. It is likely that the same neutral beam system could be used for the transformer recharge and steady state burn scenarios by varying the ion source currents and accelerator voltages. The implied electron energy continement time in the benchmark plasma is only $\sim 10$ msec so it seems likely that the temperature would be considerably higher than the benchmark value. In order to reduce the beam shinethrough (from $26 \%$ for the benchmark) it would be advisable to raise the density (lowering the beam voltage is less desirable). For $T_{e o}=12 \mathrm{keV}$, and $n_{e o}=1.2 \times 10^{19} \mathrm{~m}^{-3}$ a current of 10.3 MA (8 MA + 2.3 MA to offset the current driven by the reversed electric field of $0.01 \mathrm{~V} / \mathrm{m}$ ) can be driven by $34 \mathrm{MW}$ of $400 \mathrm{keV}$ neutral deuterium beams; the shinethrough is reduced to $14 \%$.

\subsection{Epitome of Electric Field Effects}

Detailed calculations were only performed for the NB and LH driver options. For the benchmark case $P_{C D}=24 \mathrm{MW}$ is predicted for the $L \mathrm{H}$ driver (and the fast wave options presumably are similar). For the NB, the minimum power, $P_{C D}=26-34 \mathrm{MW}$, depending on $\bar{n}_{e}$, would occur at a somewhat higher $\bar{T}_{e}$ than proposed in Table 1. These results are tabulated in Table XII. 
Table Xll

Summary of Electric Field Effects

$(E=-0.01 \mathrm{~V} / \mathrm{m})$

\begin{tabular}{|c|c|c|c|c|c|c|c|}
\hline Driver & $\left(10^{20^{n_{e}}} \mathrm{~m}^{-3}\right)$ & $\begin{array}{l}{ }^{I} \mathrm{CD} \\
(M A)\end{array}$ & $Y$ & $\begin{array}{l}\bar{T}_{e} \\
(k e v)\end{array}$ & Zeff & $\begin{array}{l}P_{C D} \\
(M W)\end{array}$ & Notes \\
\hline L.H & 0.04 & $8.0+3.0$ & 0.10 & 2 & 1.5 & 24 & $\begin{array}{l}5.0 \mathrm{GHz} ; 2.4 \\
\leqslant n_{n}<10\end{array}$ \\
\hline NB & 0.04 & $8.0+0.5$ & 0.037 & 2 & 9.0 & 45 & $\begin{array}{l}0.4 \mathrm{MeV}, D^{\circ} ; 26 \% \\
\text { shinethrough }\end{array}$ \\
\hline NB & 0.04 & $8.0+2.3$ & 0.077 & 6 & 9.0 & 26 & $\begin{array}{l}0.4 \mathrm{MeV}, \mathrm{D}^{\circ} ; 27 \% \\
\text { shinethrough }\end{array}$ \\
\hline NB & 0.06 & $8.0+2.3$ & 0.090 & 6 & 9.0 & 34 & $\begin{array}{l}0.4 \mathrm{MeV}, D^{\circ} ; 14 \% \\
\text { shinethrough }\end{array}$ \\
\hline
\end{tabular}




\section{CONCLUSIONS}

For all options we found $y \simeq 0.3-0.4$ is attainable at $\bar{T}_{e} \sim 20 \mathrm{keV}$, and, for $\bar{n}_{e} \simeq 0.7 \times 10^{20} \mathrm{~m}^{-3}$, this would permit steady state generation of 8 MA of current in INTOR $\left(R_{0}=4.9 \mathrm{~m}\right)$ with about $70-80 \mathrm{MW}$ of external power. The $\mathrm{LH}$ driver, however, does not appear to generate centrally peaked current density.

For ramp up or transtormer recharging at $\bar{T}_{e} \simeq 2-6 \mathrm{keV}$ we expect $\gamma \simeq 0.1$ for all driver options. If $E=-0.01 \mathrm{~V} / \mathrm{m}$ this should permit maintenance of a net current of $8 \mathrm{MA}$ with $\sim 25-40 \mathrm{MW}$ of external power.

\section{REFERENCES}

1. Tokamak Concept Innovations, International Atomic Energy Agency Technical Document, IAEA-TECDOC-373, IAEA, Vienna (1986).

2. J. Jacquinot, in Tokamak Startup, Heinz Knoepfel, ed., Proc. 7th Course Intern. School of Fusion Reactor Technology, July i985, Erice, Sicily, Italy, Plenum Press, NY (1986).

3. N. J. Fisch, Nucl. Fusion 21 (1981) 15.

4. Presentations at the IAEA INTOR-Related Specialists' Meeting on NonPM-86-003.

5. INTOR Phase Two A, Part II, IAEA, Vienna (1986).

6. D. A. Ehst et al., Nucl. Engineering and Design/Fusion 2 (1985) 319 .

7. D. A. Ehst et al., Nucl. Fusion 26 (1986) 461.

8. D. A. Ehst, Nucl. Fusion 19 (1979) 1369.

9. D. A. Ehst et al., J. Fusion Energy 2 (1982) 83.

10. P. Bonoli, reported in TIBER $\| 1986$ Status Report, C. D. Henning and $B$. G. Logan, eds., UCID-20863 (1986), App. A.

11. K. L. Wong and M. Ono, Nucl Fusion 23 (1983) 805.

12. J. E. Stevens et al., Bull Am. Phys. Soc. 31 (1986) 1466.

13. P. L. Andrews and D. K. Bhadra, Nuc 1. Fusion 26 (1986) 897.

14. N. J. Fisch and C. F. F. Karney, Phys. Fluids 24 (1981) 27.

15. C. F. F. Karney and N. J. Fisch, Phys. Fluids 28 (1985) 116. 
16. M. Ab dou et al., DEMO Tok amak Power Plant Study, ANL/FPP/TM-154 (1982).

17. F. W. Perkins, in FED-A, Y.-K. M. Peng and P. H. Rutherford, eds., ORNL/FEDC-83-1 (1983), P. 4-28.

18. V. L. Vdovin, INTOR Workshop, Phase 2A, Part 3, Session 13, March 1986 (unpub lished).

19. D. Moreau et al., Fast Wave Electron Current Drive, 13th European Conf. Contr. Fusion and Plasma Heating, Schliersee (1986).

20. S. C. Chiu et al., General Atomic Co., GA-A16353 (1981).

21. K. Okano et al, in Tokamak Concept Innovations, ibid., p. 209.

22. J. G. Cordey et al., Nucl. Fusion 19 (1979) 249; D. F. H. Start et al., Plas. Phys. $22(1980) 303$.

23. C. F. F. Karney and N. J. Fisch, Phys. Fluids 29 (1986) 180.

24. V. Chan et al., Phys. Fluids 29 (1986) 1900.

25. N. J. Fisch, Phys. Fluids 28 (1985) 245. 
Internal:

C. Baker

M. Billone

J. Brooks

Y. Cha

0. Chopra

R. Clemner

D. R. Diercks

D. Ehst

K. Evans

P. Finn

Y. Gohar

L. Greenwood

External:
D. Gruen

A. Hassanein

T. Hua

C. Johnson

A. Krauss

Y. Liu

B. Loomis

S. Majumdar

R. Mattas

B. Picologlou

K. Porges
C. Reed

D. Smith

H. Stevens

D. Sze

L. Turner

T. Yule

FPP Files (15)

ANL Contract File

ANL LIbrary

ANL Patent Dept.

TIS Files (5)

Manager, Chicago Operations office

S. Berk, Office of Fuston Energy

M. Cohen, Office of Fusion Energy

R. Dowling, Office of Fuston Energy

G. Haas, Office of Fuston Energy

G. R. Nardella, Office of Fuston Energy

A. Opdenaker, Office of Fusion Energy

T. Reuther, Office of Fusion Energy

P. Stone, Office of Fusion Energy

UC-20 Distribution (103)

M. Abdou, University of Cnlifornia, Los Angeles

P. Bonol1, Massachusetts Inst-1tute of Technology

L. Bromberg, Massachusetts Institute of Technology

D. Cohn, Massachusetts Institute of Technology

P. Colestock, Princeton Plasma Physics Laboratory

R. Conn, University of California, Los Angeles

M. Cox, Culham Laboratory, ENGLAND

S. Dean, Fuston Power Associates

S. Devoto, Lawrence Livermore National Laboratory

F. Engelmann, Max-Planck-Inst1tute fur Plasmaphysik, WEST GERMANY

M. Fenstermacher, Lawrence LIvermore National Laboratory

N. Fisch, Prinecton Plasma Physics Laboratory

H. Furth, Prinecton Plasma Physics Laboratory

C. Henning, Lawrence Livermore National Laboratory

D. Ignat, Princeton Plasma Physics Laboratory

J. Jacquinot, Fontenay-aux-Roses, PARIS, FRANCE

C. F. F. Karney, Princeton Plasma Physics Laboratory

G. Logan, Lawrence LIvermore National Laboratory

G. Luckhardt, Massachusetts Institute of Technology

T. K. Mau, University of California, Los Angeles

D. Mikkelsen, Prinecton Plasma Physics Laboratory

D. Moreau, Culham Laboratory, ENGLAND

K. Okano, Toshiba Corp., JAPAN

T. Okazak1, Energy Research Laboratory, JAPAN 
M. Peng, Oak Ridge National Laboratory

F. Perkins, Princeton Plasma Physics Laboratory

M. Porkolah, Massachusetts Institute of Technology

P. Rutherford, Princeton University

J. Schultz, Massachusetts Institute of Technology

D. Start, Culham Laboratory, ENGLAND

J. Stevens, Princeton Plasma Physics Laboratory

K. Thomasien, Lawrence Livermore National Laboratory

Prof. Vetter, Kernforschungszentrum KLarlsruhe und Verwaltung, GERMANY K. Yoshloka, Energy Research Laboratory, JAPAN

B 1 bliothek, Max-Planck-Inst1tute fur Plasmaphysik, WEST GERMANY

C.E.A. Library, Fontenay-aux-Roses, FRANCE

Librarian, Culham Laboratory, ENGLAND

L1brary Laboratorto Gas Ionfzati, ITALY

Thermonuclear Library, Japan Atomic Energy Research Institute, JAPAN 